Preprint typeset in JHEP style - HYPER VERSION

hep-ph/0201161

SLAC-PUB-9103

UCLA $/ 02 /$ TEP $/ 1$

January, 2002

\title{
Two-Loop Helicity Amplitudes for Gluon-Gluon Scattering in QCD and Supersymmetric Yang-Mills Theory
}

\author{
Zvi Bern,* Abilio De Freitas* \\ Department of Physics and Astronomy \\ UCLA, Los Angeles, CA 90095-1547 \\ E-mail: bern@physics.ucla.edu, freitas@physics.ucla.edu

\section{Lance Dixon ${ }^{\dagger}$} \\ Stanford Linear Accelerator Center, Stanford University \\ Stanford, CA 94309 \\ E-mail: lance@slac.stanford.edu
}

\begin{abstract}
We present the two-loop helicity amplitudes for the scattering of two gluons into two gluons in QCD, which are relevant for next-to-next-to-leading order corrections to jet production at hadron colliders. We give the results in the 't Hooft-Veltman and four-dimensional helicity variants of dimensional regularization. Summing our expressions over helicities and colors, and converting to conventional dimensional regularization, gives results in complete agreement with those of Glover, Oleari and Tejeda-Yeomans. We also present the amplitudes for $2 \rightarrow 2$ scattering in pure $N=1$ supersymmetric Yang-Mills theory.
\end{abstract}

Keywords: QCD, NNLO Computations, Jets, Hadron Colliders.

Submitted to JHEP

\footnotetext{
* Research supported by the US Department of Energy under grant DE-FG03-91ER40662.

${ }^{\dagger}$ Research supported by the US Department of Energy under grant DE-AC03-76SF00515.
} 


\section{Introduction}

For at least the next decade, the energy frontier for accelerator-based particle physics will be located at hadron colliders, the Tevatron at Fermilab and the Large Hadron Collider at CERN. At a given large momentum transfer, the most copious events at these colliders should be hadronic jets. To test the Standard Model at the shortest possible distances, therefore, the jet production cross section should be known with the highest possible precision. Existing calculations of jet production at next-to-leading order (NLO) in the strong coupling constant $\alpha_{s}[1,2,3]$ agree well with the data over a broad range of transverse momentum. Still, the NLO predictions have an uncertainty from higher order corrections, traditionally estimated from dependence on the renormalization and factorization scales, which is of order $10 \%$ or more. For very large momentum transfer the predictions can be improved by resumming threshold logarithms [4]. There are also sizable uncertainties associated with the experimental input to the parton distribution functions [5], even though global fits to the data have recently been performed [6] within an approximate next-tonext-to-leading order (NNLO) framework [7]. Nevertheless, an exact NNLO computation of jet production rates would be very welcome. Besides reducing the scale uncertainties for jet rates, the same numerical program should allow a better understanding of energy flows within jets, as a jet may consist of up to three partons at this order.

Several types of QCD amplitudes are required for a NNLO calculation of jet production at hadron colliders. Both the tree amplitudes for six external partons [8,9] and the one-loop amplitudes for five external partons [10] have been known for some time now. Recently, in a tour de force series of calculations, Anastasiou, Glover, Oleari, and Tejeda-Yeomans have provided the NNLO interferences of the two-loop amplitudes with the tree amplitudes, for all QCD four-parton processes, summed over all external helicities and colors $[11,12]$.

In this paper, we compute the $g g \rightarrow g g$ amplitudes directly at two loops in the spinor helicity formalism [13], and expose their full dependence on external colors as well. The additional helicity and color information provided here is not necessary for the main phenomenological application, NNLO jet production in collisions of unpolarized hadrons. However, it still provides several benefits:

- Jet production in collisions of polarized protons, as planned for the relativistic heavy ion collider (RHIC) at Brookhaven, may help to determine the poorly-known polarized gluon distribution in the proton [14]. Theoretical predictions of the relevant observables require scattering amplitudes for polarized partons. Currently, predictions are available through NLO [15]; the helicity amplitudes presented here are a prerequisite for improving the predictions to NNLO accuracy.

- Many formal properties of scattering amplitudes are simpler in a helicity basis and/or after color decomposition. Such properties include supersymmetry Ward identities [16], collinear limits [9, 17, 18], and high-energy behavior [19].

- Our results serve as a check of the results of ref. [12], and are useful for investigating the dependence of two-loop amplitudes on the variant of dimensional regularization used. 
Here we also present the helicity amplitudes for $g g \rightarrow g g$ scattering in pure $N=1$ supersymmetric $S U(N)$ gauge theory. Such amplitudes only differ from QCD with massless quarks in that the fermions are in the adjoint rather than the fundamental representation; yet they obey supersymmetry Ward identities [16] and are generally simpler than their QCD counterparts. They also provide useful auxiliary functions for describing the QCD results.

Several versions of dimensional regularization have been used for loop calculations in QCD, differing mainly in the number of gluon polarization states they assign in $4-2 \epsilon$ dimensions. The conventional dimensional regularization (CDR) scheme [20] assigns $D-$ $2=2-2 \epsilon$ states to all gluons, whether internal or external, virtual or real. This scheme is traditionally employed in calculations of amplitude interferences, such as ref. [12]. In the helicity approach, the number of external, observed gluon states is necessarily 2 (helicity \pm 1 ), but there is some freedom in the number of virtual gluon polarizations. The 't HooftVeltman (HV) scheme [21] contains $2-2 \epsilon$ virtual gluon states, while the four-dimensional helicity (FDH) scheme $[22,23]$ assigns 2. The FDH scheme is related to dimensional reduction $(\overline{\mathrm{DR}})[24]$ but is more compatible with the helicity method, because it allows 2 transverse dimensions in which to define helicity. Of these variants, only the FDH scheme is fully compatible with supersymmetry Ward identities for helicity amplitudes, some of which have been verified through two loops [23]. Here we work primarily in the 't HooftVeltman (HV) variant of dimensional regularization [21], but we also discuss the conversion to the CDR and FDH schemes.

Two-loop scattering amplitudes in massless QCD possess strong infrared (soft and collinear) divergences. Using dimensional regularization with $D=4-2 \epsilon$, the amplitudes generically contain poles in $\epsilon$ up to $1 / \epsilon^{4}$. However, these divergences have been organized by Catani [25] into a relatively simple form, which is completely predictable through at least order $1 / \epsilon^{2}$. We shall use Catani's formulae and color space notation to organize the $g g \rightarrow g g$ helicity amplitudes into singular terms (which do contain $\epsilon^{0}$ terms in their series expansion in $\epsilon$ ), plus finite remainders. We find that the general form of the divergences given in ref. [25] holds precisely in both the HV and FDH schemes; however, the numerical value of the coefficient $K$, which appears at order $1 / \epsilon^{2}$, differs in the FDH scheme from its value [25] in the $\mathrm{HV}$ (or $\overline{\mathrm{MS}}$ ) scheme.

The $1 / \epsilon$ poles were not predicted a priori in ref. [25] for general processes at two loops. For the $g g \rightarrow g g$ amplitude, ref. [12] computed the interference of the $1 / \epsilon$ pole terms with the tree amplitude, summed over all colors and helicities. Here we extract the full color and helicity dependence of the $1 / \epsilon$ pole terms. We find a term which is independent of color and helicity, and which agrees with that found by ref. [12] (when we use the HV scheme), plus a second term with nontrivial color-dependence, which vanishes when the color-summed interference is performed. A term with similar color structure has also been identified in contributions of one-loop factors for soft radiation to NNLO processes [26]. We shall also discuss how terms in the infrared decomposition of ref. [25] are modified, beginning at order $1 / \epsilon^{2}$, in other variants of dimensional regularization, such as the FDH scheme.

The paper is organized as follows. In section 2 we review the infrared and color struc- 
ture of one- and two-loop QCD amplitudes. In section 3 we describe the one-loop $g g \rightarrow g g$ amplitudes in a form that is valid to all orders in $\epsilon[17,27,28,29]$, and show how to expand them through $\mathcal{O}\left(\epsilon^{2}\right)$. This accuracy is required because one-loop amplitudes enter the formulae for the singular parts of two-loop amplitudes multiplied by $1 / \epsilon^{2}$. Section 3.2 shows that apart from this requirement, only finite remainder terms in the one-loop amplitudes are needed, because of cancellations with other NNLO contributions. These remainder terms are then tabulated in section 3.3.

In section 4 we describe our method for computing the two-loop amplitudes. Section 4.1 summarizes how we evaluate loop integrals, especially those that arise only in the helicity method. Some consistency checks on the results are listed in section 4.2. Section 4.3 discusses the additional singular term appearing at order $1 / \epsilon$ in the color-decomposed $g g \rightarrow g g$ amplitude, which does not contribute to the color-summed interference with the tree amplitude. The finite two-loop remainder functions in the HV scheme are then presented in section 4.4 and appendix A.

In section 5 we describe conversion of the HV results to different schemes, and the comparison with ref. [12], after our results are summed over all external colors and helicities. In section 6 and appendix B we give the two-loop amplitudes for pure $N=1$ supersymmetric Yang-Mills theory, whose finite remainders also serve as auxiliary functions for describing the QCD results. In section 7 we present our conclusions.

\section{Review of infrared and color structure}

In this section we review the structure of the infrared singularities of dimensionally regularized one- and two-loop QCD amplitudes, using Catani's color space notation [25], as a prelude to presenting the finite remainders of the one- and two-loop $g g \rightarrow g g$ amplitudes.

The process considered in this paper is

$$
g\left(-p_{1},-\lambda_{1}\right)+g\left(-p_{2},-\lambda_{2}\right) \rightarrow g\left(p_{3}, \lambda_{3}\right)+g\left(p_{4}, \lambda_{4}\right),
$$

using an "all-outgoing" convention for the external momentum $\left(p_{i}\right)$ and helicity $\left(\lambda_{i}\right)$ labeling. The Mandelstam variables are $s=\left(p_{1}+p_{2}\right)^{2}, t=\left(p_{1}+p_{4}\right)^{2}$, and $u=\left(p_{1}+p_{3}\right)^{2}$.

We work with ultraviolet renormalized amplitudes, and employ the $\overline{\mathrm{MS}}$ running coupling for QCD, $\alpha_{s}(\mu)$. The relation between the bare coupling $\alpha_{s}^{u}$ and renormalized coupling $\alpha_{s}(\mu)$, through two-loop order, is [25]

$$
\alpha_{s}^{u} \mu_{0}^{2 \epsilon} S_{\epsilon}=\alpha_{s}(\mu) \mu^{2 \epsilon}\left[1-\frac{\alpha_{s}(\mu)}{2 \pi} \frac{b_{0}}{\epsilon}+\left(\frac{\alpha_{s}(\mu)}{2 \pi}\right)^{2}\left(\frac{b_{0}^{2}}{\epsilon^{2}}-\frac{b_{1}}{2 \epsilon}\right)+\mathcal{O}\left(\alpha_{s}^{3}(\mu)\right)\right],
$$

where $\mu$ is the renormalization scale, $S_{\epsilon}=\exp [\epsilon(\ln 4 \pi+\psi(1))]$, and $\gamma=-\psi(1)=0.5772 \ldots$ is Euler's constant. The first two coefficients appearing in the beta function for QCD, or

more generally $S U(N)$ gauge theory with $N_{f}$ flavors of massless fundamental representation quarks, are

$$
b_{0}=\frac{11 C_{A}-4 T_{R} N_{f}}{6}, \quad b_{1}=\frac{17 C_{A}^{2}-\left(10 C_{A}+6 C_{F}\right) T_{R} N_{f}}{6}
$$


where $C_{A}=N, C_{F}=\left(N^{2}-1\right) /(2 N)$, and $T_{R}=1 / 2$. (Note that ref. [25] uses the notation $\beta_{0}=b_{0} /(2 \pi), \beta_{1}=b_{1} /(2 \pi)^{2}$.)

The perturbative expansion of the $g g \rightarrow g g$ amplitude is

$$
\begin{aligned}
\mathcal{M}_{g g \rightarrow g g}\left(\alpha_{s}(\mu), \mu ;\{p\}\right)=4 \pi \alpha_{s}(\mu) & {\left[\mathcal{M}_{g g \rightarrow g g}^{(0)}(\mu ;\{p\})+\right.} \\
& +\frac{\alpha_{s}(\mu)}{2 \pi} \mathcal{M}_{g g \rightarrow g g}^{(1)}(\mu ;\{p\})+ \\
& \left.+\left(\frac{\alpha_{s}(\mu)}{2 \pi}\right)^{2} \mathcal{M}_{g g \rightarrow g g}^{(2)}(\mu ;\{p\})+\mathcal{O}\left(\alpha_{s}^{3}(\mu)\right)\right],
\end{aligned}
$$

where $\mathcal{M}_{g g \rightarrow g g}^{(L)}(\mu ;\{p\})$ is the $L^{\text {th }}$ loop contribution. Equation $(2.2)$ is equivalent to the following $\overline{\mathrm{MS}}$ renormalization prescriptions at one and two loops,

$$
\begin{aligned}
& \mathcal{M}_{g g \rightarrow g g}^{(1)}=S_{\epsilon}^{-1} \mathcal{M}_{g g \rightarrow g g}^{(1) \text { unren }}-\frac{b_{0}}{\epsilon} \mathcal{M}_{g g \rightarrow g g}^{(0)}, \\
& \mathcal{M}_{g g \rightarrow g g}^{(2)}=S_{\epsilon}^{-2} \mathcal{M}_{g g \rightarrow g g}^{(2) \text { unren }}-2 \frac{b_{0}}{\epsilon} S_{\epsilon}^{-1} \mathcal{M}_{g g \rightarrow g g}^{(1) \text { unren }}+\left(\frac{b_{0}^{2}}{\epsilon^{2}}-\frac{b_{1}}{2 \epsilon}\right) \mathcal{M}_{g g \rightarrow g g}^{(0)} .
\end{aligned}
$$

The infrared divergences of renormalized one- and two-loop $n$-point amplitudes are given by [25],

$$
\begin{aligned}
& \left|\mathcal{M}_{n}^{(1)}(\mu ;\{p\})\right\rangle_{\mathrm{R} . \mathrm{S}}=\boldsymbol{I}^{(1)}(\epsilon, \mu ;\{p\})\left|\mathcal{M}_{n}^{(0)}(\mu ;\{p\})\right\rangle_{\mathrm{RS} .}+\left|\mathcal{M}_{n}^{(1) \operatorname{fin}}(\mu ;\{p\})\right\rangle_{\mathrm{RS} \mathrm{S}}, \\
& \left|\mathcal{M}_{n}^{(2)}(\mu ;\{p\})\right\rangle_{\mathrm{RS}}=\boldsymbol{I}^{(1)}(\epsilon, \mu ;\{p\})\left|\mathcal{M}_{n}^{(1)}(\mu ;\{p\})\right\rangle_{\mathrm{R} \mathrm{S}} \\
& +\boldsymbol{I}_{\mathrm{R} . \mathrm{S}}^{(2)}(\epsilon, \mu ;\{p\})\left|\mathcal{M}_{n}^{(0)}(\mu ;\{p\})\right\rangle_{\mathrm{R} . \mathrm{S} .}+\left|\mathcal{M}_{n}^{(2) \mathrm{fin}}(\mu ;\{p\})\right\rangle_{\mathrm{R} . \mathrm{S}},
\end{aligned}
$$

where the "ket" notation $\left|\mathcal{M}_{n}^{(L)}(\mu ;\{p\})\right\rangle_{\mathrm{RS}}$ indicates that the $L$-loop amplitude is treated as a vector in color space. The actual amplitude is extracted via

$$
\mathcal{M}_{n}\left(1^{a_{1}}, \ldots, n^{a_{n}}\right) \equiv\left\langle a_{1}, \ldots, a_{n} \mid \mathcal{M}_{n}\left(p_{1}, \ldots, p_{n}\right)\right\rangle
$$

where the $a_{i}$ are color indices. The subscript R. indicates that a quantity depends on the choice of renormalization scheme. The divergences of $\mathcal{M}_{n}^{(1)}$ are encoded in the color operator $\boldsymbol{I}^{(1)}$, while those of $\mathcal{M}_{n}^{(2)}$ also involve the scheme-dependent operator $\boldsymbol{I}_{\mathrm{RS} \text {. }}^{(2)}$.

In $\mathrm{QCD}$, the operator $\boldsymbol{I}^{(1)}$ is given by

$$
\boldsymbol{I}^{(1)}(\epsilon, \mu ;\{p\})=\frac{1}{2} \frac{e^{-\epsilon \psi(1)}}{\Gamma(1-\epsilon)} \sum_{i=1}^{n} \sum_{j \neq i}^{n} \boldsymbol{T}_{i} \cdot \boldsymbol{T}_{j}\left[\frac{1}{\epsilon^{2}}+\frac{\gamma_{i}}{\boldsymbol{T}_{i}^{2}} \frac{1}{\epsilon}\right]\left(\frac{\mu^{2} e^{-i \lambda_{i j} \pi}}{2 p_{i} \cdot p_{j}}\right)^{\epsilon},
$$

where $\lambda_{i j}=+1$ if $i$ and $j$ are both incoming or outgoing partons, and $\lambda_{i j}=0$ otherwise. The color charge $\boldsymbol{T}_{i}=\left\{T_{i}^{a}\right\}$ is a vector with respect to the generator label $a$, and an $S U(N)$ matrix with respect to the color indices of the outgoing parton $i$. For external gluons $T_{c b}^{a}=i f^{c a b}$, so $\boldsymbol{T}_{i}^{2}=C_{A}=N$, and

$$
\gamma_{g}=\frac{11 C_{A}-4 T_{R} N_{f}}{6}
$$


The operator $\boldsymbol{I}_{\mathrm{R} . \mathrm{S} .}^{(2)}$ is given by [25]

$$
\begin{aligned}
\boldsymbol{I}_{\mathrm{RS}}^{(2)}(\epsilon, \mu ;\{p\})= & -\frac{1}{2} \boldsymbol{I}^{(1)}(\epsilon, \mu ;\{p\})\left(\boldsymbol{I}^{(1)}(\epsilon, \mu ;\{p\})+\frac{2 b_{0}}{\epsilon}\right)+ \\
& +\frac{e^{+\epsilon \psi(1)} \Gamma(1-2 \epsilon)}{\Gamma(1-\epsilon)}\left(\frac{b_{0}}{\epsilon}+K_{\mathrm{RS}}\right) \boldsymbol{I}^{(1)}(2 \epsilon, \mu ;\{p\})+ \\
& +\boldsymbol{H}_{\mathrm{R} . \mathrm{S}}^{(2)}(\epsilon, \mu ;\{p\}),
\end{aligned}
$$

where the coefficient $K_{\mathrm{RS}}$ in either the HV or CDR schemes is given by [25]

$$
K_{\mathrm{HV}}=\left(\frac{67}{18}-\frac{\pi^{2}}{6}\right) C_{A}-\frac{10}{9} T_{R} N_{f}
$$

Although no scheme dependence was assigned to this coefficient in ref. [25], we shall find in section 5 that it is scheme dependent. The function $\boldsymbol{H}_{\mathrm{RS}}^{(2)}$ contains only single poles,

$$
\boldsymbol{H}_{\mathrm{RS}}^{(2)}(\epsilon, \mu ;\{p\})=\mathcal{O}(1 / \epsilon),
$$

but is not predicted a priori for general processes. The color- and helicity-summed matrix element $\left\langle\mathcal{M}^{(0)}\left|\boldsymbol{H}^{(2)}(\epsilon)\right| \mathcal{M}^{(0)}\right\rangle$ has previously been computed in CDR scheme for $g g \rightarrow g g$ [12] (and for some other multi-parton processes [11,30]). We shall extract the full color and helicity dependence of $\boldsymbol{H}_{\mathrm{R} . \mathrm{S}}^{(2)}(\epsilon)$ for $g g \rightarrow g g$ in the HV scheme in section 4.3 , and in the FDH scheme in section 5 .

An explicit color basis for the $g g \rightarrow g g$ amplitudes is given by

$$
\mathcal{M}_{\lambda_{1} \lambda_{2} \lambda_{3} \lambda_{4}}^{(L)}=S_{\lambda_{1} \lambda_{2} \lambda_{3} \lambda_{4}} \times \sum_{i=1}^{9} \operatorname{Tr}^{[i]} M_{\lambda_{1} \lambda_{2} \lambda_{3} \lambda_{4}}^{(L),[i]}
$$

where

$$
\begin{aligned}
& \operatorname{Tr}^{[1]}=\operatorname{tr}\left(T^{a_{1}} T^{a_{2}} T^{a_{3}} T^{a_{4}}\right), \\
& \operatorname{Tr}^{[2]}=\operatorname{tr}\left(T^{a_{1}} T^{a_{2}} T^{a_{4}} T^{a_{3}}\right), \\
& \operatorname{Tr}^{[3]}=\operatorname{tr}\left(T^{a_{1}} T^{a_{4}} T^{a_{2}} T^{a_{3}}\right), \\
& \operatorname{Tr}^{[4]}=\operatorname{tr}\left(T^{a_{1}} T^{a_{3}} T^{a_{2}} T^{a_{4}}\right), \\
& \operatorname{Tr}^{[5]}=\operatorname{tr}\left(T^{a_{1}} T^{a_{3}} T^{a_{4}} T^{a_{2}}\right), \\
& \operatorname{Tr}^{[6]}=\operatorname{tr}\left(T^{a_{1}} T^{a_{4}} T^{a_{3}} T^{a_{2}}\right), \\
& \operatorname{Tr}^{[7]}=\operatorname{tr}\left(T^{a_{1}} T^{a_{2}}\right) \operatorname{tr}\left(T^{a_{3}} T^{a_{4}}\right), \\
& \operatorname{Tr}^{[8]}=\operatorname{tr}\left(T^{a_{1}} T^{a_{3}}\right) \operatorname{tr}\left(T^{a_{2}} T^{a_{4}}\right), \\
& \operatorname{Tr}^{[9]}=\operatorname{tr}\left(T^{a_{1}} T^{a_{4}}\right) \operatorname{tr}\left(T^{a_{2}} T^{a_{3}}\right) .
\end{aligned}
$$

Here $T^{a}$ are $S U(N)$ generators in the fundamental representation, normalized according to the convention typically used in helicity amplitude calculations, $\operatorname{tr}\left(T^{a} T^{b}\right)=\delta^{a b}$. (The $T^{a}$ used in this color decomposition should not be confused with the $T_{i}^{a}$ appearing in $\boldsymbol{I}^{(1)}$, which are in the adjoint representation; nor should they be confused with the generators 
for the quark representation, which have the more "standard" normalization, $T_{R}=1 / 2$, as mentioned above.)

We have also taken the opportunity in eq. (2.15) to remove some helicity-dependent overall phases, which arise because we evaluate the amplitudes in the spinor helicity formalism [13],

$$
\begin{array}{rlrl}
S_{++++} & =i \frac{[12][34]}{\langle 12\rangle\langle 34\rangle}, & S_{-+++} & =i \frac{\langle 12\rangle\langle 14\rangle[24]}{\langle 34\rangle\langle 23\rangle\langle 24\rangle}, \\
S_{-++}=i \frac{\langle 12\rangle[34]}{[12]\langle 34\rangle}, & S_{-++} & =i \frac{\langle 13\rangle[24]}{[13]\langle 24\rangle} .
\end{array}
$$

The spinor inner products $[13,9]$ are $\langle i j\rangle=\left\langle i^{-} \mid j^{+}\right\rangle$and $[i j]=\left\langle i^{+} \mid j^{-}\right\rangle$, where $\left|i^{ \pm}\right\rangle$are massless Weyl spinors of momentum $k_{i}$, labeled with the sign of the helicity. They are anti-symmetric, with norm $|\langle i j\rangle|=|[i j]|=\sqrt{s_{i j}}$, where $s_{i j}=2 k_{i} \cdot k_{j}$. It follows that the $S_{\lambda_{1} \lambda_{2} \lambda_{3} \lambda_{4}}$ are indeed phases. They will cancel out from (and therefore may be freely omitted from) all transition probabilities involving unpolarized gluons, or circularly polarized gluons.

In the basis (2.16) for $g g \rightarrow g g$, the matrix $\boldsymbol{I}^{(1)}$ is [12]

$$
\begin{aligned}
& \boldsymbol{I}^{(1)}(\epsilon)=-\frac{e^{-\epsilon \psi(1)}}{\Gamma(1-\epsilon)}\left(\frac{1}{\epsilon^{2}}+\frac{b_{0}}{N \epsilon}\right) \times \\
& \times\left(\begin{array}{ccccccccc}
N(\mathrm{~S}+\mathrm{T}) & 0 & 0 & 0 & 0 & 0 & (\mathrm{~T}-\mathrm{U}) & 0 & (\mathrm{~S}-\mathrm{U}) \\
0 & N(\mathrm{~S}+\mathrm{U}) & 0 & 0 & 0 & 0 & (\mathrm{U}-\mathrm{T}) & (\mathrm{S}-\mathrm{T}) & 0 \\
0 & 0 & N(\mathrm{~T}+\mathrm{U}) & 0 & 0 & 0 & 0 & (\mathrm{~T}-\mathrm{S}) & (\mathrm{U}-\mathrm{S}) \\
0 & 0 & 0 & N(\mathrm{~T}+\mathrm{U}) & 0 & 0 & 0 & (\mathrm{~T}-\mathrm{S}) & (\mathrm{U}-\mathrm{S}) \\
0 & 0 & 0 & 0 & N(\mathrm{~S}+\mathrm{U}) & 0 & (\mathrm{U}-\mathrm{T}) & (\mathrm{S}-\mathrm{T}) & 0 \\
0 & 0 & 0 & 0 & 0 & N(\mathrm{~S}+\mathrm{T}) & (\mathrm{T}-\mathrm{U}) & 0 & (\mathrm{~S}-\mathrm{U}) \\
(\mathrm{S}-\mathrm{U}) & (\mathrm{S}-\mathrm{T}) & 0 & 0 & (\mathrm{~S}-\mathrm{T}) & (\mathrm{S}-\mathrm{U}) & 2 N \mathrm{~S} & 0 & 0 \\
0 & (\mathrm{U}-\mathrm{T}) & (\mathrm{U}-\mathrm{S}) & (\mathrm{U}-\mathrm{S}) & (\mathrm{U}-\mathrm{T}) & 0 & 0 & 2 N \mathrm{U} & 0 \\
(\mathrm{~T}-\mathrm{U}) & 0 & (\mathrm{~T}-\mathrm{S}) & (\mathrm{T}-\mathrm{S}) & 0 & (\mathrm{~T}-\mathrm{U}) & 0 & 0 & 2 N \mathrm{~T}
\end{array}\right)
\end{aligned}
$$

where

$$
\mathrm{S}=\left(\frac{\mu^{2}}{-s}\right)^{\epsilon}, \quad \mathrm{T}=\left(\frac{\mu^{2}}{-t}\right)^{\epsilon}, \quad \mathrm{U}=\left(\frac{\mu^{2}}{-u}\right)^{\epsilon} .
$$

A reflection identity implies that the coefficients of two color structures with reversed $T^{a_{i}}$ ordering are identical, so that

$$
M_{\lambda_{1} \lambda_{2} \lambda_{3} \lambda_{4}}^{(L),[4]}=M_{\lambda_{1} \lambda_{2} \lambda_{3} \lambda_{4}}^{(L),[3]}, \quad M_{\lambda_{1} \lambda_{2} \lambda_{3} \lambda_{4}}^{(L),[5]}=M_{\lambda_{1} \lambda_{2} \lambda_{3} \lambda_{4}}^{(L),[2]}, \quad M_{\lambda_{1} \lambda_{2} \lambda_{3} \lambda_{4}}^{(L),[6]}=M_{\lambda_{1} \lambda_{2} \lambda_{3} \lambda_{4}}^{(L),[1]}
$$

Also, due to Bose symmetry, parity, and time-reversal symmetry for the process (2.1), we only have to give results for the four helicity configurations

$$
\lambda_{1} \lambda_{2} \lambda_{3} \lambda_{4}=++++, \quad-+++, \quad--++, \quad-+-+.
$$

The tree amplitudes are given in the basis (2.16) by

$$
M_{++++}^{(0),[i]}=M_{-+++}^{(0),[i]}=0, \quad \text { for all } i,
$$




$$
\begin{array}{rlrl}
M_{\lambda_{1} \lambda_{2} \lambda_{3} \lambda_{4}}^{(0),[7]} & =M_{\lambda_{1} \lambda_{2} \lambda_{3} \lambda_{4}}^{(0),[8]}=M_{\lambda_{1} \lambda_{2} \lambda_{3} \lambda_{4}}^{(0),[9]}=0, & \text { for all } \lambda_{i} \\
M_{--++}^{(0),[1]} & =-\frac{s}{t}, & M_{--++}^{(0),[2]}=-\frac{s}{u}, & M_{--++}^{(0),[3]}=-\frac{s^{2}}{t u} \\
M_{-+-+}^{(0),[1]}=-\frac{u^{2}}{s t}, & M_{-+-+}^{(0),[2]}=-\frac{u}{s}, & M_{-+-+}^{(0),[3]}=-\frac{u}{t} .
\end{array}
$$

A typical partonic cross section requires an amplitude interference, summed over all external colors. Such interferences are evaluated in the color basis (2.16) as

$$
I_{\lambda_{1} \lambda_{2} \lambda_{3} \lambda_{4}}^{\left(L, L^{\prime}\right)} \equiv\left\langle\mathcal{M}_{\lambda_{1} \lambda_{2} \lambda_{3} \lambda_{4}}^{(L)} \mid \mathcal{M}_{\lambda_{1} \lambda_{2} \lambda_{3} \lambda_{4}}^{\left(L^{\prime}\right)}\right\rangle=\sum_{i, j=1}^{9} M_{\lambda_{1} \lambda_{2} \lambda_{3} \lambda_{4}}^{(L),[i]} \mathcal{C C}_{i j} M_{\lambda_{1} \lambda_{2} \lambda_{3} \lambda_{4}}^{\left(L^{\prime}\right),[j]}
$$

where the symmetric matrix $\mathcal{C C}_{i j} \equiv \sum_{\text {colors }} \operatorname{Tr}^{[i] *} \operatorname{Tr}^{[j]}$ is $[12,31]$

$$
\mathcal{C}=\frac{V}{N^{2}}\left(\begin{array}{ccccccccc}
C_{1} & C_{2} & C_{2} & C_{2} & C_{2} & C_{3} & N V & -N & N V \\
C_{2} & C_{1} & C_{2} & C_{2} & C_{3} & C_{2} & N V & N V & -N \\
C_{2} & C_{2} & C_{1} & C_{3} & C_{2} & C_{2} & -N & N V & N V \\
C_{2} & C_{2} & C_{3} & C_{1} & C_{2} & C_{2} & -N & N V & N V \\
C_{2} & C_{3} & C_{2} & C_{2} & C_{1} & C_{2} & N V & N V & -N \\
C_{3} & C_{2} & C_{2} & C_{2} & C_{2} & C_{1} & N V & -N & N V \\
N V & N V & -N & -N & N V & N V & N^{2} V & N^{2} & N^{2} \\
-N & N V & N V & N V & N V & -N & N^{2} & N^{2} V & N^{2} \\
N V & -N & N V & N V & -N & N V & N^{2} & N^{2} & N^{2} V
\end{array}\right),
$$

with

$$
C_{1}=N^{4}-3 N^{2}+3, \quad C_{2}=3-N^{2}, \quad C_{3}=3+N^{2}, \quad V=N^{2}-1 .
$$

The unpolarized partonic cross section is obtained from the helicity sum

$$
\bar{I}^{\left(L, L^{\prime}\right)} \equiv \sum_{\lambda_{i}= \pm 1} I_{\lambda_{1} \lambda_{2} \lambda_{3} \lambda_{4}}^{\left(L, L^{\prime}\right)}
$$

after the usual averaging over initial spins and inclusion of flux factors. For example, the helicity sum for the tree-level cross section, constructed from eq. (2.22) in either the HV or FDH scheme, is

$$
\bar{I}^{(0,0)}=16 N^{2} V\left(3-\frac{t u}{s^{2}}-\frac{u s}{t^{2}}-\frac{s t}{u^{2}}\right) .
$$

\section{One-loop amplitudes}

The one-loop amplitudes for $g g \rightarrow g g$ were first evaluated through $\mathcal{O}\left(\epsilon^{0}\right)$ as an interference with the tree amplitude in CDR scheme [32]. Later they were evaluated as helicity amplitudes in the HV and FDH schemes $[22,33]$.

Because $\boldsymbol{I}^{(1)}$ contains terms of order $1 / \epsilon^{2}$, the $\boldsymbol{I}^{(1)}\left|\mathcal{M}^{(1)}\right\rangle_{\mathrm{RS}}$ term in the infrared decomposition (2.8) of the two-loop $g g \rightarrow g g$ amplitudes requires the series expansions of the one-loop amplitudes through $\mathcal{O}\left(\epsilon^{2}\right)$. In ref. [29], using results from refs. [17, 27, 28], the 
one-loop $g g \rightarrow g g$ helicity amplitudes were presented in a representation valid to all orders in $\epsilon$, in both the HV and FDH schemes. These results can easily be rewritten in terms of integral functions whose series expansions are known to the requisite order $[34,35]$. In section 3.1 we present the all-order results in the color basis (2.16), with the normalizations implicit in eq. (2.4).

In section 3.2 , we show that the only place that terms beyond $\mathcal{O}\left(\epsilon^{0}\right)$ in the one-loop amplitudes are required in an NNLO calculation is in the infrared decomposition (2.8) of the two-loop amplitudes.

Finally, in section 3.3 we list the finite remainders of the one-loop amplitudes in the HV scheme, after the renormalization (2.5) and subtraction of infrared divergences (2.7). The corresponding finite remainder in the one-loop/one-loop NNLO interference has already been computed in CDR scheme, summed over all colors and helicities [31]. Our HV amplitude remainders lead to precisely the same result.

\subsection{All orders in $\epsilon$}

Here we present the renormalized one-loop $g g \rightarrow g g$ amplitudes in the color basis (2.16), with the normalizations implicit in eq. (2.4), in a form valid to all orders in $\epsilon$.

The first coefficient in the color basis (2.16) for $g g \rightarrow g g$ at one loop may be written in terms of "primitive" amplitudes for a gluon or quark in the loop, as [36]

$$
M_{\lambda_{1} \lambda_{2} \lambda_{3} \lambda_{4}}^{(1),[1]}(s, t, u)=N M_{\lambda_{1} \lambda_{2} \lambda_{3} \lambda_{4}}^{\text {gluon }}+N_{f} M_{\lambda_{1} \lambda_{2} \lambda_{3} \lambda_{4}}^{\text {fermin }}-\frac{b_{0}}{\epsilon} M_{\lambda_{1} \lambda_{2} \lambda_{3} \lambda_{4}}^{(0),[1]} .
$$

The remaining single-trace coefficients are obtained via crossing symmetry:

$$
\begin{array}{ll}
M_{++++}^{(1),[2]}(s, t, u)=M_{++++}^{(1),[1]}(s, u, t), & M_{++++}^{(1),[3]}(s, t, u)=M_{++++}^{(1),[1]}(u, t, s), \\
M_{-+++}^{(1),[2]}(s, t, u)=M_{-+++}^{(1),[1]}(s, u, t), & M_{-+++}^{(1),[3]}(s, t, u)=M_{-+++}^{(1),[1]}(u, t, s), \\
M_{--++}^{(1),[2]}(s, t, u)=M_{--++}^{(1),[1]}(s, u, t), & M_{-+++}^{(1),[3]}(s, t, u)=M_{-+-+}^{(1),[1]}(u, t, s), \\
M_{-+-+}^{(1),[2]}(s, t, u)=M_{-+++}^{(1),[1]}(u, s, t), & M_{-+-+}^{(1),[3]}(s, t, u)=M_{-+++}^{(1),[1]}(u, t, s),
\end{array}
$$

where appropriate analytic continuations are required to bring each function into the physical region. The double trace coefficients, to which only the gluon loops contribute, follow from a $U(1)$ decoupling identity [36]:

$$
M_{\lambda_{1} \lambda_{2} \lambda_{3} \lambda_{4}}^{(1),[7]}=M_{\lambda_{1} \lambda_{2} \lambda_{3} \lambda_{4}}^{(1),[8]}=M_{\lambda_{1} \lambda_{2} \lambda_{3} \lambda_{4}}^{(1),[9]}=\left.\frac{2}{N}\left(M_{\lambda_{1} \lambda_{2} \lambda_{3} \lambda_{4}}^{(1),[1]}+M_{\lambda_{1} \lambda_{2} \lambda_{3} \lambda_{4}}^{(1),[2]}+M_{\lambda_{1} \lambda_{2} \lambda_{3} \lambda_{4}}^{(1),[3]}\right)\right|_{N_{f}=0} .
$$

It is convenient to write the gluon and fermion loop contributions, $M_{\lambda_{1} \lambda_{2} \lambda_{3} \lambda_{4}}^{\text {gluon }}$ and $M_{\lambda_{1} \lambda_{2} \lambda_{3} \lambda_{4}}^{\text {fermin }}$, in terms of a supersymmetric decomposition into scalar, chiral $N=1$, and $N=4$ supersymmetric multiplets in the loop [37, 18]:

$$
\begin{aligned}
& M_{\lambda_{1} \lambda_{2} \lambda_{3} \lambda_{4}}^{\text {gluon }}=\left(1-\epsilon \delta_{R}\right) M_{\lambda_{1} \lambda_{2} \lambda_{3} \lambda_{4}}^{\text {scalar }}-4 M_{\lambda_{1} \lambda_{2} \lambda_{3} \lambda_{4}}^{N=1}+M_{\lambda_{1} \lambda_{2} \lambda_{3} \lambda_{4}}^{N=4}, \\
& M_{\lambda_{1} \lambda_{2} \lambda_{3} \lambda_{4}}^{\text {fermion }}=-M_{\lambda_{1} \lambda_{2} \lambda_{3} \lambda_{4}}^{\text {scalar }}+M_{\lambda_{1} \lambda_{2} \lambda_{3} \lambda_{4}}^{N=1},
\end{aligned}
$$


where $\delta_{R}=1$ for the HV scheme and $\delta_{R}=0$ for the FDH scheme.

For "maximally helicity violating" configurations, the supersymmetric components vanish by a supersymmetry Ward identity [16],

$$
M_{++++}^{N=1}=M_{-+++}^{N=1}=M_{++++}^{N=4}=M_{-+++}^{N=4}=0 .
$$

The remaining independent components are [17, 27, 29]

$$
\begin{aligned}
M_{++++}^{\text {scalar }}= & -\epsilon(1-\epsilon) \operatorname{Box}^{(8)}(s, t) \\
M_{-+++}^{\text {scalar }}= & \frac{t(u-s)}{s u} \epsilon \operatorname{Tri}^{(6)}(s)+\frac{s(u-t)}{t u} \epsilon \operatorname{Tri}^{(6)}(t)+ \\
& +\frac{t-u}{s^{2}} \epsilon \operatorname{Bub}^{(6)}(s)+\frac{s-u}{t^{2}} \epsilon \operatorname{Bub}^{(6)}(t)-\frac{s t}{2 u} \epsilon \operatorname{Box}^{(6)}(s, t)- \\
& -\epsilon(1-\epsilon) \operatorname{Box}^{(8)}(s, t), \\
M_{--++}^{\text {scalar }}= & -\frac{s-\epsilon t}{t^{2}} \operatorname{Bub}^{(6)}(t)-\epsilon(1-\epsilon) \operatorname{Box}^{(8)}(s, t), \\
M_{-+-+}^{\text {scalar }}= & -\frac{s-t}{u} \epsilon \operatorname{Tri}^{(6)}(t)-\frac{t-s}{u} \epsilon \operatorname{Tri}^{(6)}(s)+\frac{s}{u} \operatorname{Bub}^{(4)}(t)+\frac{t}{u} \operatorname{Bub}^{(4)}(s)+ \\
& +\frac{u-\epsilon t}{t^{2}} \operatorname{Bub}^{(6)}(t)+\frac{u-\epsilon s}{s^{2}} \operatorname{Bub}^{(6)}(s)-\operatorname{Tri}^{(6)}(t)-\operatorname{Tri}^{(6)}(s)- \\
& -\frac{s t}{u} \operatorname{Box}^{(6)}(s, t)-\epsilon(1-\epsilon) \operatorname{Box}^{(8)}(s, t), \\
M_{--++}^{N=1}= & -\frac{1}{2} s \epsilon \operatorname{Box}^{(6)}(s, t)-\frac{s}{2 t} \operatorname{Bub}^{(4)}(t), \\
M_{-+-+}^{N=1}= & \frac{1}{2} \frac{u}{s} \operatorname{Bub}^{(4)}(s)+\frac{1}{2} \frac{u}{t} \operatorname{Bub}^{(4)}(t)-\frac{1}{2} u(1-\epsilon) \operatorname{Box}^{(6)}(s, t), \\
M_{--++}^{N=4}= & \frac{1}{2} s^{2} \operatorname{Box}^{(4)}(s, t), \\
M_{-+-+}^{N=4}= & \frac{1}{2} u^{2} \operatorname{Box}^{(4)}(s, t) .
\end{aligned}
$$

Here $\operatorname{Bub}^{(n)}(s), \operatorname{Tri}^{(n)}(s)$ and $\operatorname{Box}^{(n)}(s, t)$ are the one-loop bubble, triangle and box scalar integrals, evaluated in $D=n-2 \epsilon$ dimensions. The bubble and box integrals are

$$
\begin{aligned}
\operatorname{Bub}^{(4)}(s) & =\frac{r_{\Gamma}}{\epsilon(1-2 \epsilon)}(-s)^{-\epsilon} \\
\operatorname{Bub}^{(6)}(s) & =-\frac{r_{\Gamma}}{2 \epsilon(1-2 \epsilon)(3-2 \epsilon)}(-s)^{1-\epsilon} \\
\operatorname{Tri}^{(4)}(s) & =-\frac{r_{\Gamma}}{\epsilon^{2}}(-s)^{-1-\epsilon} \\
\operatorname{Tri}^{(6)}(s) & =-\frac{r_{\Gamma}(-s)^{-\epsilon}}{2 \epsilon(1-2 \epsilon)(1-\epsilon)}
\end{aligned}
$$

where

$$
\begin{aligned}
r_{\Gamma} & =e^{-\epsilon \psi(1)} \frac{\Gamma(1+\epsilon) \Gamma^{2}(1-\epsilon)}{\Gamma(1-2 \epsilon)} \\
& =1-\frac{1}{2} \zeta_{2} \epsilon^{2}-\frac{7}{3} \zeta_{3} \epsilon^{3}-\frac{47}{16} \zeta_{4} \epsilon^{4}+\mathcal{O}\left(\epsilon^{5}\right),
\end{aligned}
$$


with

$$
\zeta_{s} \equiv \sum_{n=1}^{\infty} n^{-s}, \quad \zeta_{2}=\frac{\pi^{2}}{6}, \quad \zeta_{3}=1.202057 \ldots, \quad \zeta_{4}=\frac{\pi^{4}}{90},
$$

and we have kept the full dependence on $\epsilon$ in the integrals. In the $s$-channel $(s>0)$, $\epsilon$-expansions of the functions (3.10) are given by using the analytic continuation $\ln (-s) \rightarrow$ $\ln s-i \pi$.

The box integrals in various dimensions appearing in eqs. (3.7) and (3.9) are related via a dimension-shifting formula [38] valid to all orders in $\epsilon$,

$$
\begin{aligned}
& \operatorname{Box}^{(6)}(s, t)=\frac{1}{2(-1+2 \epsilon) u}\left(s t \operatorname{Box}^{(4)}(s, t)-2 t \operatorname{Tri}^{(4)}(t)-2 s \operatorname{Tri}^{(4)}(s)\right), \\
& \operatorname{Box}^{(8)}(s, t)=\frac{1}{2(-3+2 \epsilon) u}\left(s t \operatorname{Box}^{(6)}(s, t)-2 t \operatorname{Tri}^{(6)}(t)-2 s \operatorname{Tri}^{(6)}(s)\right) .
\end{aligned}
$$

Because the $D=6-2 \epsilon$ scalar box integral is completely finite as $\epsilon \rightarrow 0$, it is convenient to express the other box integrals in terms of it. This isolates all divergences to triangle and bubble integrals. To expand the six-dimensional box to higher orders in $\epsilon$, one could use an expression for $\operatorname{Box}^{(4)}(s, t)$ valid to all orders in $\epsilon$, in terms of hypergeometric functions $[39,38]$, and the dimension-shifting formula (3.13). Or one can expand the Feynman parameter integrand for $\operatorname{Box}^{(6)}(s, t)$ in $\epsilon$ directly.

In the $u$-channel $(s<0, t<0)$, where the functions are manifestly real, the expansion of the six-dimensional box through $\mathcal{O}\left(\epsilon^{2}\right)$ is $[34,35]$

$$
\begin{aligned}
\operatorname{Box}^{(6)}(s, t)=\frac{r_{\Gamma} u^{-1-\epsilon}}{2(1-2 \epsilon)}[ & \frac{1}{2}\left((V-W)^{2}+\pi^{2}\right)+ \\
& +2 \epsilon\left(\operatorname{Li}_{3}(-v)-V \operatorname{Li}_{2}(-v)-\frac{V^{3}}{3}-\frac{\pi^{2}}{2} V\right)- \\
& -2 \epsilon^{2}\left(\operatorname{Li}_{4}(-v)+W \operatorname{Li}_{3}(-v)-\frac{1}{2} V^{2} \operatorname{Li}_{2}(-v)-\right. \\
& \quad-\frac{1}{8} V^{4}-\frac{1}{6} V^{3} W+\frac{1}{4} V^{2} W^{2}-\frac{\pi^{2}}{4} V^{2}- \\
& \left.\left.\quad-\frac{\pi^{2}}{3} V W-2 \zeta_{4}\right)+(s \leftrightarrow t)\right]+\mathcal{O}\left(\epsilon^{3}\right),
\end{aligned}
$$

where

$$
v=\frac{s}{u}, \quad w=\frac{t}{u}, \quad V=\ln \left(-\frac{s}{u}\right), \quad W=\ln \left(-\frac{t}{u}\right) .
$$

In the $s$-channel $(s>0, t<0)$ an analytic continuation of the box integral yields,

$$
\begin{aligned}
\operatorname{Box}^{(6)}(s, t)= & \frac{r_{\Gamma}|s|^{-\epsilon}}{u(1-2 \epsilon)} \times \\
& \times\left\{\frac{1}{2} X^{2}+\epsilon\left(-\operatorname{Li}_{3}(-x)+X \operatorname{Li}_{2}(-x)-\frac{1}{3} X^{3}+\zeta_{3}+\frac{1}{2} Y X^{2}-\frac{1}{2} \pi^{2} X\right)-\right.
\end{aligned}
$$




$$
\begin{aligned}
& -\epsilon^{2}\left(\operatorname{Li}_{4}\left(-\frac{x}{y}\right)-\operatorname{Li}_{4}(-y)+\operatorname{Li}_{3}(-y) X+\right. \\
& \quad+\frac{1}{2} \operatorname{Li}_{2}(-x)\left(X^{2}+\pi^{2}\right)+\frac{1}{24}\left(Y^{2}+\pi^{2}\right)^{2}-\frac{1}{6} Y^{3} X+\frac{1}{4} Y^{2} X^{2}+ \\
& \left.\quad+\frac{1}{3} X^{3} Y-\frac{1}{8}\left(X^{2}+\pi^{2}\right)^{2}+\frac{\pi^{2}}{3} X Y+\frac{7}{360} \pi^{4}\right)+ \\
& +i \pi\left[X+\epsilon\left(\operatorname{Li}_{2}(-x)+Y X-\frac{1}{2} X^{2}-\frac{\pi^{2}}{6}\right)+\right. \\
& \left.\left.\quad+\epsilon^{2}\left(-\operatorname{Li}_{3}(-x)-\operatorname{Li}_{3}(-y)-\frac{1}{2} Y X^{2}+\frac{1}{6} X^{3}+\zeta_{3}\right)\right]\right\}+ \\
& +\mathcal{O}\left(\epsilon^{3}\right)
\end{aligned}
$$

where

$$
x=\frac{t}{s}, \quad y=\frac{u}{s}, \quad X=\ln \left(-\frac{t}{s}\right), \quad Y=\ln \left(-\frac{u}{s}\right) .
$$

Also define, for future use,

$$
\tilde{X}=X+i \pi, \quad \tilde{Y}=Y+i \pi .
$$

In general, both expansions of box integrals (3.14) and (3.16) will appear in eq. (2.8) for the divergences of the two-loop amplitudes.

\subsection{NNLO cancellations involving $\mathcal{M}_{g g \rightarrow g g}^{(1)}$}

The NNLO $g g \rightarrow g g$ cross section has a term proportional to the square of the one-loop amplitude, $\bar{I}^{(1,1)} \equiv\left\langle\mathcal{M}_{g g \rightarrow g g}^{(1)} \mid \mathcal{M}_{g g \rightarrow g g}^{(1)}\right\rangle$. One might expect to need the $\mathcal{O}\left(\epsilon^{2}\right)$ terms in $\mathcal{M}_{g g \rightarrow g g}^{(1)}$ here, because $\mathcal{M}_{g g \rightarrow g g}^{(1)}$ also contains $1 / \epsilon^{2}$ terms. Here we show this is not the case, for a generic NNLO calculation of an infrared-safe observable, because of cancellations against contributions involving radiation of additional partons.

If one uses the one-loop infrared decomposition (2.7), one can rewrite $\bar{I}^{(1,1)}$ as [31]

$$
\begin{aligned}
\bar{I}^{(1,1)}= & \left\langle\mathcal{M}_{g g \rightarrow g g}^{(0)}\left|\boldsymbol{I}^{(1) \dagger}(\epsilon) \boldsymbol{I}^{(1)}(\epsilon)\right| \mathcal{M}_{g g \rightarrow g g}^{(0)}\right\rangle+2 \operatorname{Re}\left\langle\mathcal{M}_{g g \rightarrow g g}^{(1) \mathrm{fin}}\left|\boldsymbol{I}^{(1)}(\epsilon)\right| \mathcal{M}_{g g \rightarrow g g}^{(0)}\right\rangle+ \\
& +\left\langle\mathcal{M}_{g g \rightarrow g g}^{(1) \mathrm{fin}} \mid \mathcal{M}_{g g \rightarrow g g}^{(1) \mathrm{fin}}\right\rangle \\
= & -\left\langle\mathcal{M}_{g g \rightarrow g g}^{(0)}\left|\boldsymbol{I}^{(1) \dagger}(\epsilon) \boldsymbol{I}^{(1)}(\epsilon)\right| \mathcal{M}_{g g \rightarrow g g}^{(0)}\right\rangle+2 \operatorname{Re}\left\langle\mathcal{M}_{g g \rightarrow g g}^{(1)}\left|\boldsymbol{I}^{(1)}(\epsilon)\right| \mathcal{M}_{g g \rightarrow g g}^{(0)}\right\rangle+ \\
& +\left\langle\mathcal{M}_{g g \rightarrow g g}^{(1) \operatorname{fin}} \mid \mathcal{M}_{g g \rightarrow g g}^{(1) \mathrm{fin}}\right\rangle .
\end{aligned}
$$

Similarly, the contribution of the two-loop/tree interference to the NNLO $g g \rightarrow g g$ cross section is

$$
\begin{aligned}
2 \operatorname{Re} \bar{I}^{(2,0)}= & 2 \operatorname{Re}\left\langle\mathcal{M}_{g g \rightarrow g g}^{(0)}\left|\boldsymbol{I}^{(2)}(\epsilon)\right| \mathcal{M}_{g g \rightarrow g g}^{(0)}\right\rangle+2 \operatorname{Re}\left\langle\mathcal{M}_{g g \rightarrow g g}^{(1)}\left|\boldsymbol{I}^{(1) \dagger}(\epsilon)\right| \mathcal{M}_{g g \rightarrow g g}^{(0)}\right\rangle+ \\
& +2 \operatorname{Re}\left\langle\mathcal{M}_{g g \rightarrow g g}^{(2) f i n} \mid \mathcal{M}_{g g \rightarrow g g}^{(0)}\right\rangle .
\end{aligned}
$$

(For clarity, we have dropped R.s. subscripts from equations in this subsection.) 
Thus the two singular terms containing the one-loop amplitude in the NNLO $g g \rightarrow g g$ cross section combine to form

$$
2 \operatorname{Re}\left\langle\mathcal{M}_{g g \rightarrow g g}^{(1)}\left|\left(\boldsymbol{I}^{(1)}(\epsilon)+\boldsymbol{I}^{(1) \dagger}(\epsilon)\right)\right| \mathcal{M}_{g g \rightarrow g g}^{(0)}\right\rangle .
$$

These terms will partially cancel, in an NNLO cross section for an infrared-safe quantity, against phase-space integration of certain terms arising from the one-loop/tree interference for the processes with one additional parton radiated (in this case, $g g \rightarrow g g g, g q \rightarrow g g q$, $g g \rightarrow g q \bar{q}$, etc.). The "radiation" terms may be written generically as

$$
2 \operatorname{Re}\left\langle\mathcal{M}_{5, \mathrm{rad}}^{(1)} \mid \mathcal{M}_{5, \mathrm{rad}}^{(0)}\right\rangle
$$

To see this NNLO cancellation, it is useful to recall the corresponding cancellation at NLO, where the singular part of the virtual correction

$$
\operatorname{Re}\left\langle\mathcal{M}_{g g \rightarrow g g}^{(0)}\left|\left(\boldsymbol{I}^{(1)}(\epsilon)+\boldsymbol{I}^{(1) \dagger}(\epsilon)\right)\right| \mathcal{M}_{g g \rightarrow g g}^{(0)}\right\rangle
$$

is cancelled by phase-space integration of the real radiation terms,

$$
\operatorname{Re}\left\langle\mathcal{M}_{5, \mathrm{rad}}^{(0)} \mid \mathcal{M}_{5, \mathrm{rad}}^{(0)}\right\rangle
$$

The singular phase-space behavior, soft or collinear, of the one-loop five-point amplitude factorizes as $[17,40,29,41]$

$$
\mathcal{M}_{5, \mathrm{rad}}^{(1)} \longrightarrow \mathcal{M}_{4}^{(1)} \mathcal{S}^{(0)}+\mathcal{M}_{4}^{(0)} \mathcal{S}^{(1)}
$$

where $\mathcal{S}^{(0)}\left(\mathcal{S}^{(1)}\right)$ represents a universal tree-level (one-loop) soft or collinear factor, which contains all the dependence on the unresolved phase-space variables that have to be integrated over. The tree-level factorization is of course

$$
\mathcal{M}_{5, \mathrm{rad}}^{(0)} \longrightarrow \mathcal{M}_{4}^{(0)} \mathcal{S}^{(0)}
$$

Using eqs. (3.25) and (3.26), one sees that the $\mathcal{M}_{4}^{(1)} \mathcal{S}^{(0)}$ terms in the singular behavior of eq. (3.22) have exactly the same form as the real NLO terms (3.24), but with $\left\langle\mathcal{M}_{4}^{(0)}\right|$ replaced by $\left\langle\mathcal{M}_{4}^{(1)}\right|$, and an overall factor of 2 from the interference. Thus the result of integrating the $\mathcal{M}_{4}^{(1)} \mathcal{S}^{(0)}$ terms in eq. (3.22) over phase space must be cancelled by the virtual NLO terms (3.23), but with the corresponding replacements, i.e. by eq. (3.21). The NLO cancellation is good to $\mathcal{O}\left(\epsilon^{0}\right)$ (after factorizing initial-state collinear singularities in the usual manner); see e.g. ref. [42]. The NNLO cancellation is at the same order, in the sense that $\mathcal{O}\left(\epsilon^{1}\right)$ and higher terms in $\mathcal{M}_{4}^{(1)}$ no longer contribute.

In summary, the only place the terms beyond $\mathcal{O}\left(\epsilon^{0}\right)$ in $\mathcal{M}_{4}^{(1)}$ are really required at NNLO is in the infrared decomposition of the two-loop amplitude. Once the two-loop finite remainders $\mathcal{M}_{4}^{(2) \text { fin }}$ are given, the higher-order terms in $\mathcal{M}_{4}^{(1)}$ are no longer needed. 


\subsection{Finite remainders}

Next we tabulate the finite remainders of the one-loop $g g \rightarrow g g$ amplitudes at $\mathcal{O}\left(\epsilon^{0}\right)$, defined by $\mathcal{M}_{g g \rightarrow g g}^{(1) \text { fin }}$ in eq. (2.7) and color decomposed into $M_{\lambda_{1} \lambda_{2} \lambda_{3} \lambda_{4}}^{(1),[i] \mathrm{fn}}$ eq. (2.15). We write, in the HV scheme,

$$
\begin{aligned}
& M_{\lambda_{1} \lambda_{2} \lambda_{3} \lambda_{4}}^{(1),[i] \mathrm{fin}}=-b_{0}\left(\ln \left(s / \mu^{2}\right)-i \pi\right) M_{\lambda_{1} \lambda_{2} \lambda_{3} \lambda_{4}}^{(0),[i]}+N a_{\lambda_{1} \lambda_{2} \lambda_{3} \lambda_{4}}^{[i]}+N_{f} c_{\lambda_{1} \lambda_{2} \lambda_{3} \lambda_{4}}^{[i]}, \quad i=1,2,3, \\
& M_{\lambda_{1} \lambda_{2} \lambda_{3} \lambda_{4}}^{(1),[i] \mathrm{fin}}=g_{\lambda_{1} \lambda_{2} \lambda_{3} \lambda_{4}}^{[i]}+\frac{N_{f}}{N} h_{\lambda_{1} \lambda_{2} \lambda_{3} \lambda_{4}}^{[i]}, \quad i=7,8,9
\end{aligned}
$$

and the $M^{(1),[i] \text { fin }}$ for $i=4,5,6$ follow from eq. (2.20). The one-loop $U(1)$ decoupling identity (3.3) implies that

$$
g_{\lambda_{1} \lambda_{2} \lambda_{3} \lambda_{4}}^{[i]}=2\left(a_{\lambda_{1} \lambda_{2} \lambda_{3} \lambda_{4}}^{[1]}+a_{\lambda_{1} \lambda_{2} \lambda_{3} \lambda_{4}}^{[2]}+a_{\lambda_{1} \lambda_{2} \lambda_{3} \lambda_{4}}^{[3]}\right), \quad i=7,8,9 .
$$

For the,++++-+++ , and --++ helicity configurations, Bose symmetry under exchange of legs 3 and $4(t \leftrightarrow u)$ implies that

$$
\begin{aligned}
& a_{\lambda_{1} \lambda_{2} \lambda_{3} \lambda_{4}}^{[2]}(s, t, u)=a_{\lambda_{1} \lambda_{2} \lambda_{3} \lambda_{4}}^{[1]}(s, u, t), \\
& c_{\lambda_{1} \lambda_{2} \lambda_{3} \lambda_{4}}^{[2]}(s, t, u)=c_{\lambda_{1} \lambda_{2} \lambda_{3} \lambda_{4}}^{[1]}(s, u, t) .
\end{aligned}
$$

For the ++++ helicity amplitude, the independent remainder functions $a, c, g$ and $h$ are

$$
\begin{aligned}
& a_{++++}^{[1]}=-\frac{1}{6}, \\
& a_{++++}^{[3]}=-\frac{1}{6}, \\
& c_{++++}^{[1]}=\frac{1}{6}, \\
& c_{++++}^{[3]}=\frac{1}{6}, \\
& h_{++++}^{[7]}=h_{++++}^{[8]}=h_{++++}^{[9]}=0 .
\end{aligned}
$$

For -+++ , they are

$$
\begin{aligned}
& a_{-+++}^{[1]}=-\frac{y^{2}}{6 x}, \\
& a_{-+++}^{[3]}=-\frac{1}{6 x y}, \\
& c_{-+++}^{[1]}=\frac{y^{2}}{6 x}, \\
& c_{-+++}^{[3]}=\frac{1}{6 x y}, \\
& h_{-+++}^{[7]}=h_{-+++}^{[8]}=h_{-+++}^{[9]}=0,
\end{aligned}
$$

where $x$ and $y$ are defined in eq. (3.17). 
For --++ , they are

$$
\begin{aligned}
a_{--++}^{[1]}= & -\frac{1}{2 x}\left(\tilde{X}^{2}+\pi^{2}\right)+\frac{67}{18 x}, \\
a_{--++}^{[3]}= & -\frac{(1-x y)^{2}}{4 x y}\left((X-Y)^{2}+\pi^{2}\right)-\left(\frac{11}{6 y}-\frac{x-y}{2}\right) \tilde{X}+\frac{67}{36 x y}+\frac{1}{4}+ \\
& +\{t \leftrightarrow u\}, \\
c_{--++}^{[1]}= & -\frac{5}{9 x}, \\
c_{--++}^{[3]}= & -\frac{x^{2}}{4}\left((X-Y)^{2}+\pi^{2}\right)+\frac{1}{6}\left(2 \frac{x^{2}}{y}+x+5 y\right) \tilde{X}-\frac{29}{36}-\frac{5}{9} \frac{x}{y}+ \\
& +\{t \leftrightarrow u\}, \\
h_{--++}^{[7]}= & h_{--++}^{[8]}=h_{--++}^{[9]}=\frac{2}{3 y} \tilde{X}+\{t \leftrightarrow u\} .
\end{aligned}
$$

For -+-+ , the required functions are

$$
\begin{aligned}
& a_{-+-+}^{[1]}=-\frac{(1-x y)^{2}}{2 x y^{2}}\left(\tilde{X}^{2}+\pi^{2}\right)-\left(\frac{4}{3} y-\frac{x}{2}+\frac{1}{y}\right) \tilde{X}+\frac{67}{18} \frac{y^{2}}{x}+\frac{1}{2}, \\
& a_{-+-+}^{[2]}=-\frac{y}{2}\left(\tilde{Y}^{2}+\pi^{2}\right)+\frac{11}{6} y \tilde{Y}+\frac{67}{18} y, \\
& a_{-+-+}^{[3]}=-\frac{y}{2 x}\left((X-Y)^{2}+\pi^{2}\right)+\frac{11}{6} \frac{y}{x} \tilde{Y}+\frac{67}{18} \frac{y}{x}, \\
& c_{-+-+}^{[1]}=\left(\frac{x}{2 y^{2}}-\frac{1}{4}\right)\left(\tilde{X}^{2}+\pi^{2}\right)+\frac{x(x-y)+5}{6 y} \tilde{X}-\frac{(3-2 y)(3 x-2 y)}{18 x}, \\
& c_{-+-+}^{[2]}=-\frac{y}{3} \tilde{Y}-\frac{5}{9} y, \\
& c_{-+-+}^{[3]}=-\frac{y}{3 x} \tilde{Y}-\frac{5}{9} \frac{y}{x}, \\
& h_{-+-+}^{[7]}=h_{-+-+}^{[8]}=h_{-+-+}^{[9]}=\frac{2}{3} y \tilde{X}+\frac{2}{3} \frac{y^{2}}{x} \tilde{Y} .
\end{aligned}
$$

The contribution of the one-loop finite remainders to the NNLO $g g \rightarrow g g$ cross section is

$$
\bar{I}^{(1,1) \mathrm{fin}} \equiv \sum_{\lambda_{i}= \pm 1}\left\langle\mathcal{M}_{\lambda_{1} \lambda_{2} \lambda_{3} \lambda_{4}}^{(1) \mathrm{fin}} \mid \mathcal{M}_{\lambda_{1} \lambda_{2} \lambda_{3} \lambda_{4}}^{(1) \mathrm{fin}}\right\rangle
$$

Using the color sum matrix $\mathcal{C C}_{i j}$ in eq. (2.24), the color and helicity sum in $\bar{I}^{(1,1) \text { fin }}$ may be evaluated in terms of the above explicit expressions (3.27)-(3.51) for $M_{\lambda_{1} \lambda_{2} \lambda_{3} \lambda_{4}}^{(1)[\text {. It repro- }}$ duces precisely the finite remainder function Finite $(s, t, u)$ given in eq. (3.22) of ref. [31] for the corresponding quantity evaluated in CDR scheme; the HV/CDR scheme difference for $\bar{I}^{(1,1)}$ has been completely absorbed into the first two of the three terms in eq. (3.19). 

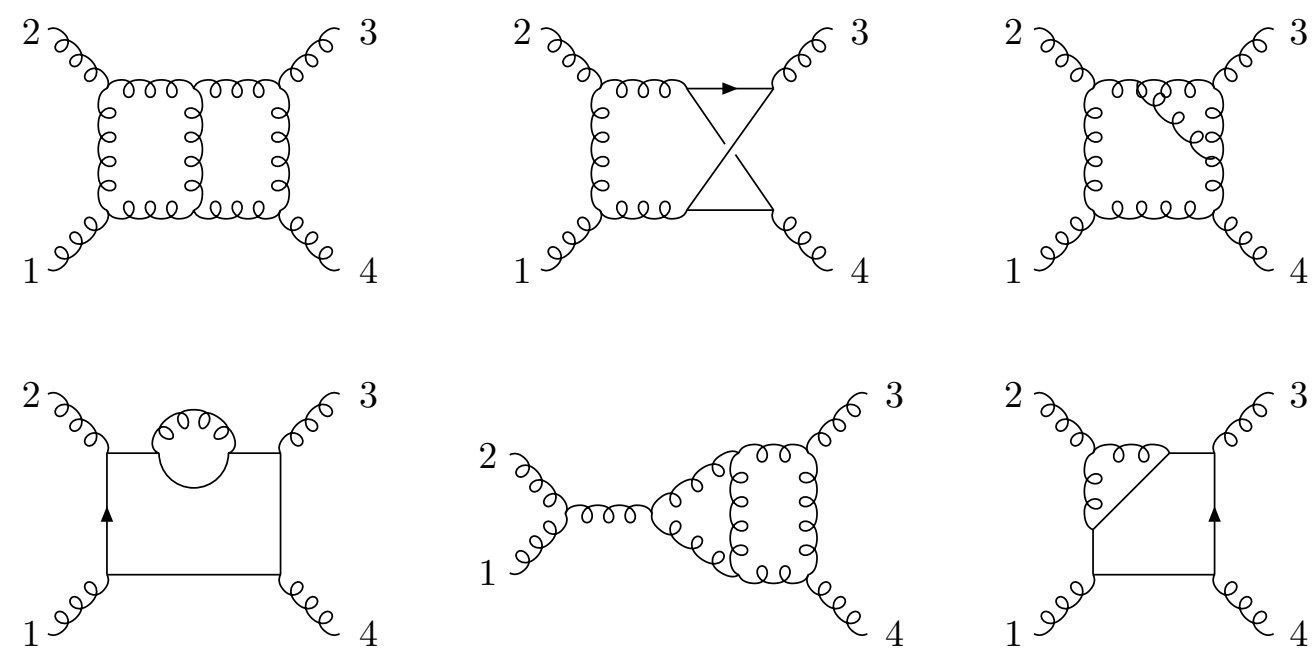

Figure 1: Some of the two-loop diagrams for $g g \rightarrow g g$.

\section{Two-loop amplitudes and finite remainders}

A generic sample of two-loop Feynman diagrams for $g g \rightarrow g g$ is shown in figure 1 . However, we did not evaluate the diagrams directly. Instead we computed the unitarity cuts in various channels, working to all orders in the dimensional regularization parameter $\epsilon=(4-D) / 2[43,17,37,18]$. Essentially we followed the approach first employed at two loops for the pure gluon four-point amplitude with all helicities identical [34] and for $N=4$ supersymmetric amplitudes [44]. These amplitudes were simple enough that a compact expression for the integrand could be given. The fermion loop contributions with all helicities identical are about as simple [23]. For other helicity configurations, the integrands become rather complicated. We therefore used the general integral reduction algorithms developed for the all-massless four-point topologies [45, 46, 47, 48], in order to reduce the loop integrals to a minimal basis of master integrals. To efficiently incorporate polarization vectors of gluons with definite helicity requires some minor extensions of these techniques, which we now discuss.

\subsection{Tensor loop integrals}

Here we discuss techniques for evaluating the loop integrals required for the two-loop amplitudes for $g g \rightarrow g g$ and related processes, with an emphasis on the additional types of integrands encountered in the helicity amplitude method.

In calculating a typical two-loop scattering amplitude in QCD, a large number of two-loop integrals are encountered. The most complicated topologies are the planar and non-planar double box integrals, displayed in figure 2, which are given by

$$
\begin{aligned}
& \mathcal{I}_{4}^{\mathrm{P}}[\mathcal{P}](s, t) \equiv \\
& \quad \equiv \int \frac{d^{D} p}{(2 \pi)^{D}} \frac{d^{D} q}{(2 \pi)^{D}} \frac{\mathcal{P}}{p^{2} q^{2}(p+q)^{2}\left(p-k_{1}\right)^{2}\left(p-k_{1}-k_{2}\right)^{2}\left(q-k_{4}\right)^{2}\left(q-k_{3}-k_{4}\right)^{2}},
\end{aligned}
$$




$$
\begin{aligned}
& \mathcal{I}_{4}^{\mathrm{NP}}[\mathcal{P}](s, t) \equiv \\
& \quad \equiv \int \frac{d^{D} p}{(2 \pi)^{D}} \frac{d^{D} q}{(2 \pi)^{D}} \frac{\mathcal{P}}{p^{2} q^{2}(p+q)^{2}\left(p-k_{1}\right)^{2}\left(q-k_{2}\right)^{2}\left(p+q+k_{3}\right)^{2}\left(p+q+k_{3}+k_{4}\right)^{2}} .
\end{aligned}
$$

Here $p$ and $q$ are the loop momenta, and $k_{i}, i=1,2,3,4$, are the external (outgoing) momenta. $\mathcal{P}$ is a polynomial (or tensor) in the loop momenta $p$ and $q$, which accompanies the scalar propagator factors shown in the figure. It is generated by the numerator algebra of the Feynman diagram, or unitarity cut, that is being evaluated.
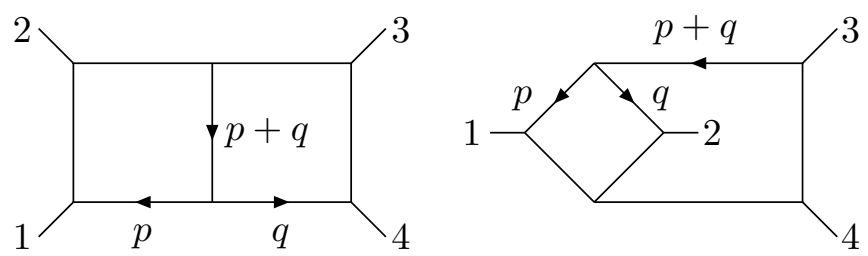

Figure 2: The planar and non-planar double box integrals.

In the interference method, as recently applied to two-loop QED and QCD scattering amplitudes $[35,11,12,30]$, one sums over all external polarization states in $D$ dimensions. In this case, $\mathcal{P}$ can only depend on the loop momenta, $p$ and $q$, and external momenta, $k_{i}$. By Lorentz invariance, this dependence is only through scalar products,

$$
\mathcal{P}_{\text {interf. }}=\mathcal{P}\left(p^{2}, p \cdot q, q^{2}, p \cdot k_{i}, q \cdot k_{i}\right) .
$$

In contrast, in the helicity amplitude method [13] used in the present paper - and previously applied to two-loop amplitudes in $N=4$ super-Yang-Mills theory [44], $g g \rightarrow g g$ for identical helicities in pure Yang-Mills theory [34], and the QCD processes $g g \rightarrow \gamma \gamma$ [49] and $\gamma \gamma \rightarrow \gamma \gamma \quad[50]-\mathcal{P}$ also depends on the polarization vectors $\varepsilon_{i}$ for the external gluons. We take $D>4$ in the calculation, i.e., $\epsilon<0$, in order to have two transverse dimensions in which to define helicities. Because the polarization vectors are intrinsically four-dimensional, their Lorentz products with the loop momenta distinguish between the four-dimensional and $(-2 \epsilon)$-dimensional components of $p$ and $q$. Write

$$
p^{\mu}=p_{[4]}^{\mu}+\vec{\lambda}_{p}, \quad q^{\mu}=q_{[4]}^{\mu}+\vec{\lambda}_{q},
$$

where $p_{[4]}, q_{[4]}$ are the four-dimensional components and $\vec{\lambda}_{p}, \vec{\lambda}_{q}$ are the $(-2 \epsilon)$-dimensional components. We use the Minkowski metric with signature $(1,-1,-1,-1, \ldots)$, and write

$$
p^{2}=p_{[4]}^{2}-\lambda_{p}^{2}, \quad q^{2}=q_{[4]}^{2}-\lambda_{q}^{2}, \quad(p+q)^{2}=\left(p_{[4]}+q_{[4]}\right)^{2}-\lambda_{p+q}^{2},
$$

where $\lambda_{p}^{2} \equiv \vec{\lambda}_{p} \cdot \vec{\lambda}_{p} \geq 0, \quad \lambda_{p+q}^{2} \equiv\left(\vec{\lambda}_{p}+\vec{\lambda}_{q}\right)^{2}=\lambda_{p}^{2}+\lambda_{q}^{2}+2 \vec{\lambda}_{p} \cdot \vec{\lambda}_{q}$. Then the generic polynomial encountered in the helicity amplitude method has the form

$$
\mathcal{P}_{\text {hel. }}=\mathcal{P}\left(p^{2}, p \cdot q, q^{2}, p \cdot k_{i}, q \cdot k_{i} ; \lambda_{p}^{2}, \lambda_{q}^{2}, \lambda_{p+q}^{2}, \varepsilon_{i} \cdot p, \varepsilon_{i} \cdot q\right) .
$$


We rely on reduction algorithms developed to handle general tensor integrals for the all-massless planar [45] and non-planar [47] double boxes, and related topologies such as the pentabox [46]. These algorithms were derived using integration by parts [51] and (for the non-planar double box) Lorentz invariance [48] identities, which act in the space of integrals with $\mathcal{P}=1$, but with the scalar propagators raised to arbitrary integer powers $\nu_{i}$. For example, for the planar double box topology one considers

$$
I_{4}^{\mathrm{P}}\left(\nu_{1}, \nu_{2}, \nu_{3}, \ldots, \nu_{7}\right) \equiv(4 \pi)^{D} \int \frac{d^{D} p}{(2 \pi)^{D}} \frac{d^{D} q}{(2 \pi)^{D}} \prod_{i=1}^{7} \frac{1}{\left(p_{i}^{2}\right)^{\nu_{i}}},
$$

where

$$
\begin{aligned}
& p_{1}=q, \quad p_{2}=q-k_{3}-k_{4}, \quad p_{3}=p, \quad p_{4}=p-k_{1}-k_{2}, \\
& p_{5}=p-k_{1}, \quad p_{6}=p+q, \quad p_{7}=q-k_{4},
\end{aligned}
$$

and $\nu_{i} \in\{0,1,2, \ldots\}$. The reduction algorithms reduce any such integral to a linear combination of simpler "boundary" integrals, where at least one of the $\nu_{i}$ vanishes, plus one or two master integrals with the same topology. All told, there are 10 different master integrals for the massless $2 \rightarrow 2$ processes [45, 47, 46].

Given an integral with $\mathcal{P}$ of the form (4.3) or (4.6), it is simple to convert it to integrals of the form (4.7) using Schwinger parametrization [52]. For the scalar integrals, using

$$
\frac{1}{p_{i}^{2}}=\int_{0}^{\infty} d t_{i} \exp \left(-t_{i} p_{i}^{2}\right)
$$

and performing the $p$ and $q$ integrals, leads to

$$
\begin{aligned}
I_{4}^{X}(1,1, \ldots, 1) & =\prod_{i=1}^{7} \int_{0}^{\infty} d t_{i}[\Delta(T)]^{-D / 2} \exp \left[-\frac{Q_{X}\left(s, t, t_{i}\right)}{\Delta(T)}\right], \\
\Delta(T) & \equiv T_{p} T_{q}+T_{p} T_{p q}+T_{q} T_{p q}
\end{aligned}
$$

where $X$ labels the topology of the integral (P, NP, etc.). In eq. (4.11), $T_{p}, T_{q}, T_{p q}$ are the sums of Schwinger parameters along the lines carrying loop momenta $p, q, p+q$, respectively. Equivalently, they are Schwinger parameters for the two-loop vacuum graph obtained by omitting all the external lines, as shown in figure 3. For the planar double box integral, with propagators numbered by eq. (4.8), the $T_{a}$ are given by

$$
T_{p}=t_{3}+t_{4}+t_{5}, \quad T_{q}=t_{1}+t_{2}+t_{7}, \quad T_{p q}=t_{6}
$$

The quantity $Q_{X}$ is more cumbersome. For the planar double box integral, its expression is

$$
Q_{\mathrm{P}}\left(s, t, t_{i}\right)=-s\left[t_{1} t_{2}\left(t_{3}+t_{4}+t_{5}\right)+t_{3} t_{4}\left(t_{1}+t_{2}+t_{7}\right)+t_{6}\left(t_{1}+t_{3}\right)\left(t_{2}+t_{4}\right)\right]-t t_{5} t_{6} t_{7} .
$$

However, the precise form of $Q_{X}$ will not matter in the following. 


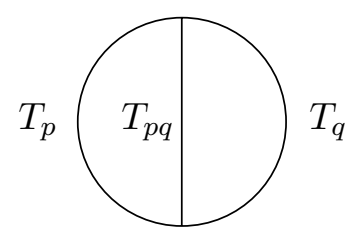

Figure 3: The two-loop vacuum graph obtained by omitting external momenta, and its three Schwinger parameters, which are relevant for general two-loop integrals containing $v \cdot p, v \cdot q, \vec{\lambda}_{p}$, and $\vec{\lambda}_{q}$.

If a polynomial $\mathcal{P}$ is present, Schwinger parametrization converts it to a polynomial in the Schwinger parameters, along with inverse powers of $\Delta$. Then the more general parametrization,

$$
\frac{1}{\left(p_{i}^{2}\right)^{\nu_{i}}}=\frac{1}{\Gamma\left(\nu_{i}\right)} \int_{0}^{\infty} d t_{i} t_{i}^{\nu_{i}-1} \exp \left(-t_{i} p_{i}^{2}\right)
$$

can be used to rewrite Schwinger parameter monomials as integrals of the form (4.7), typically in shifted dimensions, $D \rightarrow D+2 n, n=0,1,2, \ldots$ (to account for the inverse powers of $\Delta$ ). (Shifted-dimension integrals pose no problem; equations for them can be found by rewriting the factor $\Delta^{-D / 2}$ in eq. (4.10) as

$$
\Delta^{-D / 2}=\left(T_{p} T_{q}+T_{p} T_{p q}+T_{q} T_{p q}\right) \times \Delta^{-(D+2) / 2},
$$

and reducing the latter, shifted-dimension representation.) In principle, this approach gives a prescription to handle any polynomial in the loop momentum, for either the interference or helicity method.

However, as the degree $\sum_{i} \nu_{i}$ increases, the number of integrals of the form (4.7) grows rapidly, and the reduction algorithm can become rather time-consuming. We have found it useful to instead use simple algebraic relations, e.g. for the planar double box integral,

$$
\begin{aligned}
2 q \cdot k_{4} & =p_{1}^{2}-p_{7}^{2}, \quad 2 q \cdot k_{3}=p_{7}^{2}-p_{2}^{2}+2 k_{3} \cdot k_{4}, \\
2 p \cdot k_{1} & =p_{3}^{2}-p_{5}^{2}, \quad 2 p \cdot k_{2}=p_{5}^{2}-p_{4}^{2}+2 k_{1} \cdot k_{2}, \\
2 p \cdot q & =p_{6}^{2}-p_{1}^{2}-p_{3}^{2}
\end{aligned}
$$

to quickly reduce integrals with polynomials of the form $\mathcal{P}_{\text {interf. }}$ to a relatively small set of "irreducible" integrals for each topology, plus boundary integrals generated when the $p_{i}^{2}$ factors cancel propagators. Of course the "irreducible" integrals are only irreducible with respect to (4.16), and not with respect to the integration-by-parts and Lorentz identities. We compute the "irreducible" integrals once and store them.

For the planar double box, eq. (4.16) and momentum conservation, $k_{1}+k_{2}+k_{3}+k_{4}=0$, show that the "irreducible" monomials needed to generate all $\mathcal{P}_{\text {interf. }}$ are

$$
\mathcal{P}_{\text {interf. }}^{\text {P, irred }}(m, n)=\left(2 q \cdot k_{1}\right)^{m}\left(2 p \cdot k_{4}\right)^{n}, \quad m+n \leq 6 .
$$

The restriction on the sum of $m$ and $n$ comes from gauge theory - at most six powers of the loop momentum can appear in the Feynman diagram numerator algebra. The 
planar double box also has a symmetry, reflection about the central vertical line, so that $\mathcal{P}_{\text {interf. }}(m, n)=\mathcal{P}_{\text {interf. }}(n, m)$. This leaves only 16 integrals (4.17) to store. Not all of the highest degree integrals actually appear in the amplitude. For the non-planar box, we store the integrals of the monomials,

$$
\mathcal{P}_{\text {interf. }}^{\mathrm{NP}, \text { irred }}(m, n)=\left(2(p+q) \cdot k_{1}\right)^{m}\left(2 p \cdot k_{4}\right)^{n}, \quad m+n \leq 6 .
$$

We also store those "irreducible" boundary integrals with six (instead of seven) propagators; these monomials are generated by three independent factors. For example, for the planar double box boundary integral obtained by setting $\nu_{3} \rightarrow 0$, one requires

$$
\left(2 q \cdot k_{1}\right)^{m}\left(2 p \cdot k_{4}\right)^{n}\left(2 p \cdot k_{1}\right)^{r}, \quad m+n+r \leq 5 .
$$

For the helicity amplitude approach, the more general loop momentum polynomial $\mathcal{P}_{\text {hel. }}$ given in eq. (4.6) requires a bit more work before the above method can be used. Consider the product $\varepsilon_{i} \cdot p$. Because $\varepsilon_{i}^{\mu}$ is a four-dimensional vector, this can also be written as $\varepsilon_{i} \cdot p_{[4]}$. We can expand $p_{[4]}^{\mu}$ in terms of a basis of four different four-dimensional vectors. Because of momentum conservation, there are only three independent external momenta, but we can use the Levi-Civita tensor to construct a fourth one,

$$
v^{\mu} \equiv \varepsilon_{\nu_{1} \nu_{2} \nu_{3}}^{\mu} k_{1}^{\nu_{1}} k_{2}^{\nu_{2}} k_{3}^{\nu_{3}}
$$

Then

$$
p_{[4]}^{\mu} \equiv c_{1}^{p} k_{1}^{\mu}+c_{2}^{p} k_{2}^{\mu}+c_{3}^{p} k_{3}^{\mu}+c_{v}^{p} v^{\mu},
$$

where

$$
\begin{aligned}
c_{1}^{p} & =\frac{1}{2 s u}\left[-t\left(2 p \cdot k_{1}\right)+u\left(2 p \cdot k_{2}\right)+s\left(2 p \cdot k_{3}\right)\right], \\
c_{2}^{p} & =\frac{1}{2 s t}\left[t\left(2 p \cdot k_{1}\right)-u\left(2 p \cdot k_{2}\right)+s\left(2 p \cdot k_{3}\right)\right], \\
c_{3}^{p} & =\frac{1}{2 t u}\left[t\left(2 p \cdot k_{1}\right)+u\left(2 p \cdot k_{2}\right)-s\left(2 p \cdot k_{3}\right)\right], \\
c_{v}^{p} & =-\frac{4}{s t u} \varepsilon_{\mu \nu_{1} \nu_{2} \nu_{3}} p^{\mu} k_{1}^{\nu_{1}} k_{2}^{\nu_{2}} k_{3}^{\nu_{3}}=-\frac{4}{s t u} v \cdot p .
\end{aligned}
$$

Thus we can write

$$
\varepsilon_{i} \cdot p=c_{1}^{p} \varepsilon_{i} \cdot k_{1}+c_{2}^{p} \varepsilon_{i} \cdot k_{2}+c_{3}^{p} \varepsilon_{i} \cdot k_{3}+c_{v}^{p} \varepsilon_{i} \cdot v .
$$

This equation, and the analogous one for $\varepsilon_{i} \cdot q$, reduce the problem of handling helicity amplitude polynomials (4.6) to those of the form

$$
\tilde{\mathcal{P}}_{\text {hel. }}=\mathcal{P}\left(p^{2}, p \cdot q, q^{2}, p \cdot k_{i}, q \cdot k_{i} ; \lambda_{p}^{2}, \lambda_{q}^{2}, \lambda_{p+q}^{2}, v \cdot p, v \cdot q\right),
$$

where $v \cdot p$ and $v \cdot q$ come from the $c_{v}$ coefficients.

The effect of inserting factors of $v \cdot p$ and $v \cdot q$ into the integral is very similar to inserting factors involving the $(-2 \epsilon)$-dimensional components of the loop momenta, $\vec{\lambda}_{p}$ and $\vec{\lambda}_{q}$. In either case, shifts of $p$ and $q$ by amounts proportional to the external momenta 
$k_{i}$, as required to perform the Gaussian integrals over $p$ and $q$, have no effect, because $v \cdot k_{i}=\lambda_{j} \cdot k_{i}=0$. Thus both types of factors result only in polynomials in the "vacuum graph" Schwinger parameters $T_{a}$.

Insertions of $\lambda_{p}^{2}, \lambda_{q}^{2}$ and $\lambda_{p+q}^{2}$ may be handled by differentiating the $(-2 \epsilon)$-dimensional part of the (Wick rotated) integral,

$$
\int d^{-2 \epsilon} \lambda_{p} d^{-2 \epsilon} \lambda_{q} \exp \left[-\lambda_{p}^{2} T_{p}-\lambda_{q}^{2} T_{q}-\lambda_{p+q}^{2} T_{p q}\right] \propto \Delta^{\epsilon},
$$

with respect to $T_{p}, T_{q}$ and $T_{p q}$. They lead to parameter insertions such as

$$
\begin{aligned}
\lambda_{p}^{2} & \rightarrow-\epsilon \frac{T_{q}+T_{p q}}{\Delta}, \\
\lambda_{p+q}^{2} & \rightarrow-\epsilon \frac{T_{p}+T_{q}}{\Delta}, \\
\left(\lambda_{p}^{2}\right)^{2} & \rightarrow-\epsilon(1-\epsilon) \frac{\left(T_{q}+T_{p q}\right)^{2}}{\Delta^{2}}, \\
\lambda_{p}^{2} \lambda_{q}^{2} & \rightarrow \frac{\epsilon^{2}}{\Delta}-\epsilon(1-\epsilon) \frac{T_{p q}^{2}}{\Delta^{2}}, \\
\left(\lambda_{p}^{2}\right)^{2} \lambda_{q}^{2} & \rightarrow \epsilon(1-\epsilon)\left[\epsilon \frac{T_{q}+T_{p q}}{\Delta^{2}}-(2-\epsilon) \frac{T_{p q}^{2}\left(T_{q}+T_{p q}\right)}{\Delta^{3}}\right], \\
\lambda_{p}^{2} \lambda_{q}^{2} \lambda_{p+q}^{2} & \rightarrow \epsilon(1-\epsilon)\left[\epsilon \frac{T_{p}+T_{q}+T_{p q}}{\Delta^{2}}+(2-\epsilon) \frac{T_{p} T_{q} T_{p q}}{\Delta^{3}}\right] .
\end{aligned}
$$

Similarly, the polynomials in $v \cdot p$ and $v \cdot q$ are easily parametrized:

$$
\begin{aligned}
(v \cdot p)^{2} & \rightarrow \frac{s t u}{8} \frac{T_{q}+T_{p q}}{\Delta}, \\
(v \cdot(p+q))^{2} & \rightarrow \frac{s t u}{8} \frac{T_{p}+T_{q}}{\Delta}, \\
(v \cdot p)^{4} & \rightarrow 3\left(\frac{s t u}{8}\right)^{2} \frac{\left(T_{q}+T_{p q}\right)^{2}}{\Delta^{2}}, \\
(v \cdot p)^{2}(v \cdot q)^{2} & \rightarrow\left(\frac{s t u}{8}\right)^{2}\left[\frac{1}{\Delta}+3 \frac{T_{p q}^{2}}{\Delta^{2}}\right], \\
(v \cdot p)^{4}(v \cdot q)^{2} & \rightarrow\left(\frac{s t u}{8}\right)^{3}\left[3 \frac{T_{q}+T_{p q}}{\Delta^{2}}+15 \frac{T_{p q}^{2}\left(T_{q}+T_{p q}\right)}{\Delta^{3}}\right], \\
(v \cdot p)^{2}(v \cdot q)^{2}(v \cdot(p+q))^{2} & \rightarrow\left(\frac{s t u}{8}\right)^{3}\left[3 \frac{T_{p}+T_{q}+T_{p q}}{\Delta^{2}}-15 \frac{T_{p} T_{q} T_{p q}}{\Delta^{3}}\right] .
\end{aligned}
$$

These equations apply to any two-loop integral, independent of the external momenta. They also apply in the presence of any additional numerator factor of the form $f\left(p \cdot k_{i}, q \cdot k_{i}\right)$, since cross contractions are forbidden by the orthogonality of $v$ to the $k_{i}$.

Finally, polynomials in $v \cdot p, v \cdot q$ can be related to those in $\vec{\lambda}_{i}$, using the expansion (4.21). For example,

$$
p^{2}+\lambda_{p}^{2}=p_{[4]} \cdot p_{[4]}=s c_{1}^{p} c_{2}^{p}+t c_{2}^{p} c_{3}^{p}+u c_{1}^{p} c_{3}^{p}-\frac{1}{4} s t u\left(c_{v}^{p}\right)^{2}
$$


or

$$
\lambda_{p}^{2}=-\frac{4}{s t u}(v \cdot p)^{2}+\hat{\mathcal{P}}_{p}
$$

where

$$
\hat{\mathcal{P}}_{p} \equiv-p^{2}+s c_{1}^{p} c_{2}^{p}+t c_{2}^{p} c_{3}^{p}+u c_{1}^{p} c_{3}^{p}
$$

Similarly,

$$
\begin{aligned}
\lambda_{q}^{2} & =-\frac{4}{s t u}(v \cdot q)^{2}+\hat{\mathcal{P}}_{q}, \\
\lambda_{p+q}^{2} & =-\frac{4}{s t u}(v \cdot(p+q))^{2}+\hat{\mathcal{P}}_{p q},
\end{aligned}
$$

where

$$
\begin{aligned}
\hat{\mathcal{P}}_{q} & \equiv-q^{2}+s c_{1}^{q} c_{2}^{q}+t c_{2}^{q} c_{3}^{q}+u c_{1}^{q} c_{3}^{q} \\
\hat{\mathcal{P}}_{p q} & \equiv-(p+q)^{2}+s\left(c_{1}^{p}+c_{1}^{q}\right)\left(c_{2}^{p}+c_{2}^{q}\right)+t\left(c_{2}^{p}+c_{2}^{q}\right)\left(c_{3}^{p}+c_{3}^{q}\right)+u\left(c_{1}^{p}+c_{1}^{q}\right)\left(c_{3}^{p}+c_{3}^{q}\right) .
\end{aligned}
$$

Note that $\hat{\mathcal{P}}_{p}, \hat{\mathcal{P}}_{q}$ and $\hat{\mathcal{P}}_{p q}$ only contain the types of Lorentz products which already appear in $\mathcal{P}_{\text {interf. }}$.

Because eqs. (4.26) and (4.27) are so similar, and taking into account the relations between the integrands, we can solve for the additional "new" integrals required for the helicity method, in terms of the "old" integrals needed for the interference method. For example, for a general function $f\left(p \cdot k_{i}, q \cdot k_{i}\right)$, we have

$$
\begin{aligned}
\int \lambda_{p}^{2} f & =-\epsilon \frac{8}{s t u} \int(v \cdot p)^{2} f=-\frac{2 \epsilon}{1-2 \epsilon} \int \hat{\mathcal{P}}_{p} f \\
\int \lambda_{p+q}^{2} f & =-\epsilon \frac{8}{s t u} \int(v \cdot(p+q))^{2} f=-\frac{2 \epsilon}{1-2 \epsilon} \int \hat{\mathcal{P}}_{p q} f \\
\int\left(\lambda_{p}^{2}\right)^{2} f & =-\frac{\epsilon(1-\epsilon)}{3}\left(\frac{8}{s t u}\right)^{2} \int(v \cdot p)^{4} f=-\frac{4 \epsilon(1-\epsilon)}{(1-2 \epsilon)(3-2 \epsilon)} \int \hat{\mathcal{P}}_{p}^{2} f, \\
\int \lambda_{p}^{2} \lambda_{q}^{2} f & =-\frac{4 \epsilon(1-\epsilon)}{(1-2 \epsilon)(3-2 \epsilon)} \int \hat{\mathcal{P}}_{p} \hat{\mathcal{P}}_{q} f+\frac{\epsilon}{3-2 \epsilon} \int \frac{f}{\Delta}, \\
\int(v \cdot p)^{2}(v \cdot q)^{2} f & =\left(\frac{s t u}{4}\right)^{2}\left[\frac{3}{(1-2 \epsilon)(3-2 \epsilon)} \int \hat{\mathcal{P}}_{p} \hat{\mathcal{P}}_{q} f+\frac{\epsilon}{3-2 \epsilon} \int \frac{f}{\Delta}\right], \\
\int\left(\lambda_{p}^{2}\right)^{2} \lambda_{q}^{2} f & =-\frac{8 \epsilon(1-\epsilon)(2-\epsilon)}{(1-2 \epsilon)(3-2 \epsilon)(5-2 \epsilon)} \int \hat{\mathcal{P}}_{p}^{2} \hat{\mathcal{P}}_{q} f+ \\
& +\frac{4 \epsilon(1-\epsilon)}{(3-2 \epsilon)(5-2 \epsilon)} \int \frac{\hat{\mathcal{P}}_{p} f}{\Delta} .
\end{aligned}
$$

A factor of $1 / \Delta$ indicates that a shift of the dimension of the integral is required: $D \rightarrow D+2$, $\epsilon \rightarrow \epsilon-1$ ( $\epsilon$ 's in prefactors should be shifted as well).

In practice, we used eqs. (4.29) and (4.31) to eliminate $v \cdot p$ and $v \cdot q$ in favor of $\lambda_{p}^{2}$, $\lambda_{q}^{2}$ and $\lambda_{p+q}^{2}$ in the loop momentum polynomial. We used equations like (4.33) to compute 
the "irreducible" monomials including the $\vec{\lambda}_{i}$, which we then stored. E.g., for the planar double box integral, we stored values for

$$
\begin{array}{r}
\mathcal{P}_{\text {hel. }}^{\mathrm{P}, \text { irred }}\left(l_{1}, l_{2}, l_{3}, m, n\right)=\left(\lambda_{p}^{2}\right)^{l_{1}}\left(\lambda_{q}^{2}\right)^{l_{2}}\left(\lambda_{p+q}^{2}\right)^{l_{3}}\left(2 q \cdot k_{1}\right)^{m}\left(2 p \cdot k_{4}\right)^{n}, \\
2 l_{1}+2 l_{2}+2 l_{3}+m+n \leq 6 .
\end{array}
$$

Having reduced all the tensor loop integrals in the amplitudes to a linear combination of master integrals, the next step is to expand the master integrals in a Laurent series in $\epsilon$, beginning at order $1 / \epsilon^{4}$, using results from refs. [53, 54, 45, 47, 46]. Many of these master integral expansions are given in terms of Nielsen functions [55], usually denoted by $S_{n, p}(x)$. However, it is straightforward [56] to express the results solely in terms of polylogarithms [57],

$$
\begin{aligned}
\operatorname{Li}_{n}(x) & =\sum_{i=1}^{\infty} \frac{x^{i}}{i^{n}}=\int_{0}^{x} \frac{d t}{t} \operatorname{Li}_{n-1}(t), \\
\operatorname{Li}_{2}(x) & =-\int_{0}^{x} \frac{d t}{t} \ln (1-t),
\end{aligned}
$$

with $n=2,3,4$. The analytic properties of the non-planar double box integrals appearing in the amplitudes are somewhat intricate [34,54]; there is no Euclidean region in any of the three kinematic channels, $s, t$ or $u$. So we do not attempt to give a crossing-symmetric representation, but instead quote all our results in the physical $s$-channel $(s>0 ; t, u<0)$ for the $g g \rightarrow g g$ kinematics (2.1).

\subsection{Checks on results}

We performed a number of consistency checks on the amplitudes to ensure their reliability:

1. As a check of gauge invariance, we verified that the amplitudes vanish when a gluon polarization vector is replaced with a longitudinal one.

2. The agreement of the explicitly computed infrared divergences with the expected form (2.8) provides a stringent check on the amplitudes. Most of the master integrals contain divergent as well as finite terms, so the finite remainders are checked indirectly in this way.

3. Using supersymmetry Ward identities [16], we evaluated the identical-helicity case, including fermion loops [23], by relating it to the already known identical-helicity pure-glue $g g \rightarrow g g$ amplitude [34]. The integration in ref. [34] was done by a completely different technique, thus checking the programs and integration methods used to obtain the general helicity cases.

4. As described in more detail at the end of section 5, we compared our results for $g g \rightarrow g g$ to those of ref. [12]. The interference of the two-loop $g g \rightarrow g g$ helicity amplitudes with the tree amplitudes, after summing over all external helicities and colors, and accounting for the different schemes used (HV vs. CDR), agrees precisely with the calculation of ref. [12]. 


\section{$4.3 \boldsymbol{H}^{(2)}$ operator}

As mentioned in section 2, the function $\boldsymbol{H}_{\mathrm{RSS}}^{(2)}(\epsilon)$, which contains only $1 / \epsilon$ poles, has not been predicted a priori for general processes. However, there is accumulating evidence from explicit calculations $[11,12,30]$ in the CDR scheme that the color- and helicity-summed matrix element $\left\langle\mathcal{M}^{(0)}\left|\boldsymbol{H}^{(2)}(\epsilon)\right| \mathcal{M}^{(0)}\right\rangle$ is a sum of terms for each external colored leg in the process, namely

$$
\left\langle\mathcal{M}^{(0)}\left|\boldsymbol{H}^{(2)}(\epsilon)\right| \mathcal{M}^{(0)}\right\rangle=\frac{e^{-\epsilon \psi(1)}}{4 \epsilon \Gamma(1-\epsilon)}\left(n_{g} H_{g}^{(2)}+n_{q} H_{q}^{(2)}\right)\left\langle\mathcal{M}^{(0)} \mid \mathcal{M}^{(0)}\right\rangle,
$$

where $n_{g}$ is the number of external gluons, and $n_{q}$ is the number of external quarks plus anti-quarks, with

$$
\begin{aligned}
H_{g}^{(2)}= & \left(\frac{\zeta_{3}}{2}+\frac{11}{144} \pi^{2}+\frac{5}{12}\right) N^{2}+\left(-\frac{\pi^{2}}{72}-\frac{89}{108}\right) N N_{f}-\frac{N_{f}}{4 N}+\frac{5}{27} N_{f}^{2} \\
H_{q}^{(2)}= & \left(\frac{7}{4} \zeta_{3}-\frac{11}{96} \pi^{2}+\frac{409}{864}\right) N^{2}+\left(-\frac{1}{4} \zeta_{3}-\frac{\pi^{2}}{96}-\frac{41}{108}\right)+\left(-\frac{3}{2} \zeta_{3}+\frac{\pi^{2}}{8}-\frac{3}{32}\right) \frac{1}{N^{2}}+ \\
& +\left(\frac{\pi^{2}}{48}-\frac{25}{216}\right) \frac{N^{2}-1}{N} N_{f} .
\end{aligned}
$$

Note that $H_{g}^{(2)}$ and $H_{q}^{(2)}$ are constants, independent of the kinematic variables.

We find that the full color and helicity dependence of $\boldsymbol{H}_{\mathrm{R.S}}^{(2)}(\epsilon)$ for $g g \rightarrow g g$ is the sum of two terms,

$$
\boldsymbol{H}^{(2)}(\epsilon)=\frac{e^{-\epsilon \psi(1)}}{4 \epsilon \Gamma(1-\epsilon)}\left(\frac{\mu^{2}}{-s}\right)^{2 \epsilon}\left(4 H_{g}^{(2)} \mathbf{1}+\hat{\boldsymbol{H}}^{(2)}\right)
$$

where

$$
\hat{\boldsymbol{H}}^{(2)}=-4 \ln \left(\frac{-s}{-t}\right) \ln \left(\frac{-t}{-u}\right) \ln \left(\frac{-u}{-s}\right) \times\left[\boldsymbol{T}_{1} \cdot \boldsymbol{T}_{2}, \boldsymbol{T}_{2} \cdot \boldsymbol{T}_{3}\right],
$$

with $\ln ((-s) /(-t)) \rightarrow \ln s-\ln (-t)-i \pi$ in the $s$-channel, etc. (The overall factor of $\left(\mu^{2} /(-s)\right)^{2 \epsilon}$ is a choice of convention, because $\left(\mu^{2} /(-s)\right)^{2 \epsilon}-1$ is of order $\epsilon$. Including it cleans up the finite remainder $\mathcal{M}_{g g \rightarrow g g}^{(2) \text { fin }}$ a bit.) The first term in the sum is proportional to the identity matrix in both helicity and color spaces. In the HV scheme, $H_{g}^{(2)}$ is given by precisely the same value (4.38) found in the CDR scheme [12]. The value in the FDH scheme is different; see eq. (5.9).

The second term in eq. (4.40) is also independent of the helicity configuration, but it is a nontrivial commutator matrix in color space. (The possibility of nontrivial color structure in $\boldsymbol{H}^{(2)}(\epsilon)$ was pointed out in ref. [25].) Indeed, it vanishes when sandwiched between tree amplitudes, after performing the color sum,

$$
\left\langle\mathcal{M}^{(0)}\left|\hat{\boldsymbol{H}}^{(2)}\right| \mathcal{M}^{(0)}\right\rangle \propto\left\langle\mathcal{M}^{(0)}\left|\left(\boldsymbol{T}_{1} \cdot \boldsymbol{T}_{2} \boldsymbol{T}_{2} \cdot \boldsymbol{T}_{3}-\boldsymbol{T}_{2} \cdot \boldsymbol{T}_{3} \boldsymbol{T}_{1} \cdot \boldsymbol{T}_{2}\right)\right| \mathcal{M}^{(0)}\right\rangle=0,
$$

using hermiticity of the $\boldsymbol{T}_{i}$. Equation (4.42) ensures that the result (4.40) is perfectly compatible with the previous color-summed results (4.37).

Actually, in the course of the $q \bar{q} \rightarrow q \bar{q}$ calculation, the authors of ref. [11] evaluated the matrix element

$$
\left\langle\mathcal{M}_{q \bar{q}^{\prime} \rightarrow q \bar{q}^{\prime}}^{(0)}\left|\hat{\boldsymbol{H}}^{(2)}\right| \mathcal{M}_{q \bar{q} \rightarrow q^{\prime} \bar{q}^{\prime}}^{(0)}\right\rangle,
$$


relevant for the interference term in identical-quark scattering. Because the color structures for the $s$-channel and $t$-channel tree amplitudes, $\mathcal{M}_{q \bar{q} \rightarrow q^{\prime} \bar{q}^{\prime}}^{(0)}$ and $\mathcal{M}_{q \bar{q}^{\prime} \rightarrow q \bar{q}^{\prime}}^{(0)}$, are different, eq. (4.42) does not apply, and the non-vanishing CDR result is completely consistent with our HV result eq. (4.41), including all normalization factors [58].

We find that the commutator term is the same in FDH scheme as well. Note that it can be rewritten as a "triple product" in color space, because

$$
\left[\boldsymbol{T}_{1} \cdot \boldsymbol{T}_{2}, \boldsymbol{T}_{2} \cdot \boldsymbol{T}_{3}\right]=i f_{a b c} T_{1}^{a} T_{2}^{b} T_{3}^{c} .
$$

This form of the color operator has previously appeared in analysis of the contributions of one-loop factors for soft radiation (i.e., $\mathcal{S}^{(1)}$ in eq. (3.25)) at NNLO [26]. This fact, and

the lack of scheme dependence for $\hat{\boldsymbol{H}}^{(2)}$, leads one to suspect that it arises from soft, not collinear, virtual contributions. The $1 / \epsilon$ divergence would presumably cancel against the contributions discussed in ref. [26], in a color-resolved approach to a NNLO computation. In a fully color-summed approach, however, such contributions should cancel individually, thanks to eq. (4.42).

The factorization of $\hat{\boldsymbol{H}}^{(2)}$ in eq. (4.41) into a product of kinematic and color factors is clearly an accident of having exactly four external colored partons. Color conservation, $\boldsymbol{T}_{1}+\boldsymbol{T}_{2}+\boldsymbol{T}_{3}+\boldsymbol{T}_{4}=0$ in the color-space notation, implies that there are only three independent $\boldsymbol{T}_{i} \cdot \boldsymbol{T}_{j}$ factors, say $\boldsymbol{T}_{1} \cdot \boldsymbol{T}_{2}, \boldsymbol{T}_{2} \cdot \boldsymbol{T}_{3}$, and $\boldsymbol{T}_{1} \cdot \boldsymbol{T}_{3}$. But their sum is a $c$-number,

$$
\boldsymbol{T}_{1} \cdot \boldsymbol{T}_{2}+\boldsymbol{T}_{2} \cdot \boldsymbol{T}_{3}+\boldsymbol{T}_{1} \cdot \boldsymbol{T}_{3}=\frac{1}{2}\left[\boldsymbol{T}_{4}^{2}-\boldsymbol{T}_{1}^{2}-\boldsymbol{T}_{2}^{2}-\boldsymbol{T}_{3}^{2}\right]
$$

hence there is only one independent commutator. (A similar argument holds in the $f_{a b c} T_{i}^{a} T_{j}^{b} T_{k}^{c}$ representation [26].) For three or less external colored partons, all such structures vanish; whereas for five or more partons there are multiple independent ones.

\subsection{Finite remainders}

The two-loop finite remainders are defined in eq. (2.8) and are color decomposed into $M_{\lambda_{1} \lambda_{2} \lambda_{3} \lambda_{4}}^{(2),[] \mathrm{in}}$ eq. (2.15). Their dependence on the renormalization scale $\mu, N$ and $N_{f}$ may be extracted as

$$
\begin{array}{rlr}
M_{\lambda_{1} \lambda_{2} \lambda_{3} \lambda_{4}}^{(2),[i] \mathrm{fin}}= & -\left[b_{0}^{2}\left(\ln \left(s / \mu^{2}\right)-i \pi\right)^{2}+b_{1}\left(\ln \left(s / \mu^{2}\right)-i \pi\right)\right] M_{\lambda_{1} \lambda_{2} \lambda_{3} \lambda_{4}}^{(0),[i]}- \\
& -2 b_{0}\left(\ln \left(s / \mu^{2}\right)-i \pi\right) M_{\lambda_{1} \lambda_{2} \lambda_{3} \lambda_{4}}^{(1),[i \mathrm{fin}}+ \\
& +N^{2} A_{\lambda_{1} \lambda_{2} \lambda_{3} \lambda_{4}}^{[i]}+B_{\lambda_{1} \lambda_{2} \lambda_{3} \lambda_{4}}^{[i]}+N N_{f} C_{\lambda_{1} \lambda_{2} \lambda_{3} \lambda_{4}}^{[i]}+\frac{N_{f}}{N} D_{\lambda_{1} \lambda_{2} \lambda_{3} \lambda_{4}}^{[i]}+ \\
& +N_{f}^{2} E_{\lambda_{1} \lambda_{2} \lambda_{3} \lambda_{4}}^{[i]}+\frac{N_{f}^{2}}{N^{2}} F_{\lambda_{1} \lambda_{2} \lambda_{3} \lambda_{4}}^{[i]}, & \\
M_{\lambda_{1} \lambda_{2} \lambda_{3} \lambda_{4}}^{(2),[i] \mathrm{fin}}= & -2 b_{0}\left(\ln \left(s / \mu^{2}\right)-i \pi\right) M_{\lambda_{1} \lambda_{2} \lambda_{3} \lambda_{4}}^{(1),[i] \mathrm{fin}}+ & \\
& +N G_{\lambda_{1} \lambda_{2} \lambda_{3} \lambda_{4}}^{[i]}+N_{f} H_{\lambda_{1} \lambda_{2} \lambda_{3} \lambda_{4}}^{[i]}+\frac{N_{f}^{2}}{N} I_{\lambda_{1} \lambda_{2} \lambda_{3} \lambda_{4}}^{[i]}, \quad i=7,3,9,9 .
\end{array}
$$

The $\mu$-dependence is a consequence of renormalization group invariance. The tree and one-loop functions, $M_{\lambda_{1} \lambda_{2} \lambda_{3} \lambda_{4}}^{(0),[i]}$ and $M_{\lambda_{1} \lambda_{2} \lambda_{3} \lambda_{4}}^{(1),[i] \mathrm{f}}$, are given in eq. (2.22) and eqs. (3.27)-(3.51), respectively, while $b_{0}$ and $b_{1}$ are given in eq. (2.3). 
The coefficient functions $A, B, C, D, E, F, G, H, I$, which depend only on the Mandelstam variables, obey several relations. Group theory (e.g. $U(1)$ decoupling identities) implies that the $G$ functions are not independent of the others,

$$
\begin{aligned}
& G_{\lambda_{1} \lambda_{2} \lambda_{3} \lambda_{4}}^{[7]}=2\left(A_{\lambda_{1} \lambda_{2} \lambda_{3} \lambda_{4}}^{[1]}+A_{\lambda_{1} \lambda_{2} \lambda_{3} \lambda_{4}}^{[2]}+A_{\lambda_{1} \lambda_{2} \lambda_{3} \lambda_{4}}^{[3]}\right)-B_{\lambda_{1} \lambda_{2} \lambda_{3} \lambda_{4}}^{[3]}, \\
& G_{\lambda_{1} \lambda_{2} \lambda_{3} \lambda_{4}}^{[8]}=2\left(A_{\lambda_{1} \lambda_{2} \lambda_{3} \lambda_{4}}^{[1]}+A_{\lambda_{1} \lambda_{2} \lambda_{3} \lambda_{4}}^{[2]}+A_{\lambda_{1} \lambda_{2} \lambda_{3} \lambda_{4}}^{[3]}\right)-B_{\lambda_{1} \lambda_{2} \lambda_{3} \lambda_{4}}^{[1]}, \\
& G_{\lambda_{1} \lambda_{2} \lambda_{3} \lambda_{4}}^{[9]}=2\left(A_{\lambda_{1} \lambda_{2} \lambda_{3} \lambda_{4}}^{[1]}+A_{\lambda_{1} \lambda_{2} \lambda_{3} \lambda_{4}}^{[2]}+A_{\lambda_{1} \lambda_{2} \lambda_{3} \lambda_{4}}^{[3]}\right)-B_{\lambda_{1} \lambda_{2} \lambda_{3} \lambda_{4}}^{[2]},
\end{aligned}
$$

and that the sum of the $B$ coefficients vanishes,

$$
B_{\lambda_{1} \lambda_{2} \lambda_{3} \lambda_{4}}^{[3]}=-B_{\lambda_{1} \lambda_{2} \lambda_{3} \lambda_{4}}^{[1]}-B_{\lambda_{1} \lambda_{2} \lambda_{3} \lambda_{4}}^{[2]} .
$$

As at one loop, for the,++++-+++ , and --++ helicity configurations, Bose symmetry under exchange of legs 3 and $4(t \leftrightarrow u)$ implies further relations,

$$
\begin{aligned}
X_{\lambda_{1} \lambda_{2} \lambda_{3} \lambda_{4}}^{[2]}(s, t, u) & =X_{\lambda_{1} \lambda_{2} \lambda_{3} \lambda_{4}}^{[1]}(s, u, t), & & X \in\{A, B, C, D, E, F\}, \\
Y_{\lambda_{1} \lambda_{2} \lambda_{3} \lambda_{4}}^{[9]}(s, t, u) & =Y_{\lambda_{1} \lambda_{2} \lambda_{3} \lambda_{4}}^{[8]}(s, u, t), & & Y \in\{G, H, I\} .
\end{aligned}
$$

In appendix A, we give the explicit forms for the independent finite remainder functions appearing in eqs. (4.46) and (4.47). For the two complicated helicity configurations, --++ and -+-+ , these functions also involve auxiliary functions, $A^{\mathrm{SYM},[i]}$ and $B^{\mathrm{SYM},[i]}$, which will be presented in appendix B. The latter functions also serve as the finite remainders for $g g \rightarrow g g$ in $N=1$ super-Yang-Mills theory, as discussed in section 6 .

\section{Scheme conversion at two loops}

The preceding helicity amplitudes were presented in the HV variant of dimensional regularization and renormalization. As mentioned in the introduction, the HV scheme contains $D-2=2-2 \epsilon$ virtual (unobservable) gluon states, and 2 external (observable) gluon states. However, it is possible to alter the number of virtual states. In the FDH scheme [22, 23], one adjusts the number of virtual gluon states to be 2, matching the number of external states, and also matching the number of fermionic degrees of freedom in a supersymmetric theory. This scheme is quite similar to dimensional reduction $(\overline{\mathrm{DR}})[24]$.

Dimensional reduction is usually thought of as having $D<4$, i.e., $\epsilon>0$, and contains $D-2=2-2 \epsilon$ gluon states, plus $2 \epsilon$ scalar states, for a total of 2 bosonic states. On the other hand, the helicity of a particle is its angular momentum eigenvalue for a rotation in the two-dimensional plane normal to its momentum vector. If $D$ is less than four, this plane does not exist, making the definition of helicity obscure. The FDH scheme can be regarded as an analytic continuation of $\overline{\mathrm{DR}}$ to $D>4$, to make it compatible with helicity amplitudes. No scalars are required, however. For both the HV and FDH schemes, helicity amplitudes with fermions and gluons are computed in the same fashion, with $D$-dimensional loop momenta and four-dimensional gluon polarization vectors (see section 4.1). In performing 
the algebra leading to the loop-momentum polynomial $\mathcal{P}$, when the trace of the Minkowski metric is encountered, one sets

$$
\begin{aligned}
\eta_{\mu}^{\mu} & \equiv D_{s} \equiv 4-2 \epsilon \delta_{R} \\
\delta_{R} & = \begin{cases}1, & \text { HV scheme } \\
0, & \text { FDH scheme }\end{cases}
\end{aligned}
$$

This procedure is gauge invariant because the terms proportional to $D_{s}$ are related to loops containing scalar fields in the adjoint representation [23]. We allow $\delta_{R}$ to be arbitrary below, although only the HV and FDH cases in eq. (5.2) seem well motivated.

The CDR scheme has $D_{s}-2=2-2 \epsilon$ virtual gluon states, just as in the HV scheme; but in addition there are $D-2=2-2 \epsilon$ external gluon states. To convert from the HV to the CDR scheme within the helicity method, one could in principle compute additional amplitudes where some external states have $\epsilon$-helicities (explicit polarization vectors that point into the extra $(-2 \epsilon)$-dimensions) [59]. Since the CDR result is already available via the interference method [12], we have not done that computation. Instead we shall check the conversion between schemes expected from experience at one loop.

A given scheme has implications for regularization of both ultraviolet and infrared singularities. These implications have been discussed extensively at one loop [22, 33, 60]. Let us first consider the ultraviolet situation. Renormalization by modified minimal subtraction, as in eqs. (2.5) and (2.6), leads to different renormalized coupling constants, related by finite shifts. In the class of schemes we are considering, the ultraviolet behavior only depends on the number of virtual gluon states. Thus the CDR and HV schemes imply the same coupling constant, the standard $\overline{\mathrm{MS}}$ coupling, $\alpha_{s}(\mu)$. The FDH and $\overline{\mathrm{DR}}$ schemes also are the same in the ultraviolet (the ability to accommodate helicity, and the sign of $\epsilon$, are irrelevant here), and so they both define the $\overline{\mathrm{DR}}$ coupling, $\alpha_{s}^{\overline{\mathrm{DR}}}(\mu)$.

To shift from either pair of schemes to the other, in the ultraviolet, amounts to using the following relations between coupling constants [61,33], recently improved to two-loop accuracy [23],

$$
\begin{aligned}
\alpha_{s}^{\overline{\mathrm{DR}}}(\mu)=\alpha_{s}(\mu)[1 & +\frac{C_{A}}{6} \frac{\alpha_{s}(\mu)}{2 \pi}+\frac{11 C_{A}^{2}-9 C_{F} T_{R} N_{f}}{18}\left(\frac{\alpha_{s}(\mu)}{2 \pi}\right)^{2}+ \\
& \left.+\mathcal{O}\left(\left[\alpha_{s}(\mu)\right]^{3}\right)\right] \\
\alpha_{s}(\mu)=\alpha_{s}^{\overline{\mathrm{DR}}}(\mu)[ & {\left[1-\frac{C_{A}}{6} \frac{\alpha_{s}^{\overline{\mathrm{DR}}}(\mu)}{2 \pi}-\frac{10 C_{A}^{2}-9 C_{F} T_{R} N_{f}}{18}\left(\frac{\alpha_{s}^{\overline{\mathrm{DR}}}(\mu)}{2 \pi}\right)^{2}+\right.} \\
& \left.+\mathcal{O}\left(\left[\alpha_{s}^{\overline{\mathrm{DR}}}(\mu)\right]^{3}\right)\right]
\end{aligned}
$$

(Recall that the three-loop running coupling enters into any NNLO computation. The three-loop beta-function coefficient $b_{2}$ in $\overline{\mathrm{DR}}$ differs from the value in $\overline{\mathrm{MS}}$ [62], but it can be obtained simply from the coupling shift (5.3) [23].) For completeness, we give the two-loop relation [23] between the $\overline{\mathrm{MS}}$ coupling and that defined by an arbitrary value of 
$\delta_{R}$,

$$
\begin{aligned}
\alpha_{s}^{\delta_{R}}(\mu)=\alpha_{s}(\mu)[1 & +\frac{C_{A}}{6}\left(1-\delta_{R}\right) \frac{\alpha_{s}(\mu)}{2 \pi}+ \\
& +\left(\frac{C_{A}^{2}}{36}\left(1-\delta_{R}\right)^{2}+\frac{7 C_{A}^{2}-6 C_{F} T_{R} N_{f}}{12}\left(1-\delta_{R}\right)\right)\left(\frac{\alpha_{s}(\mu)}{2 \pi}\right)^{2}+ \\
& \left.+\mathcal{O}\left(\left[\alpha_{s}(\mu)\right]^{3}\right)\right], \\
\alpha_{s}(\mu)=\alpha_{s}^{\delta_{R}}(\mu)[1 & -\frac{C_{A}}{6}\left(1-\delta_{R}\right) \frac{\alpha_{s}^{\delta_{R}}(\mu)}{2 \pi}+ \\
& +\left(\frac{C_{A}^{2}}{36}\left(1-\delta_{R}\right)^{2}-\frac{7 C_{A}^{2}-6 C_{F} T_{R} N_{f}}{12}\left(1-\delta_{R}\right)\right)\left(\frac{\alpha_{s}^{\delta_{R}}(\mu)}{2 \pi}\right)^{2}+ \\
& \left.+\mathcal{O}\left(\left[\alpha_{s}^{\delta_{R}}(\mu)\right]^{3}\right)\right]
\end{aligned}
$$

which reduces to eqs. (5.3) and (5.4) for $\delta_{R}=0$. Inserting the appropriate coupling relation into the perturbative expansion of the amplitude (2.4) leads to simple, finite ultraviolet conversion relations between renormalized amplitudes $\mathcal{M}_{n}^{(L)}$.

Because the ultraviolet shifts are so simple to implement, in the rest of this paper (with one exception to be discussed below) we take the ultraviolet scheme to be the same as the infrared scheme. That is, when we report results for $\mathcal{M}_{g g \rightarrow g g}^{(L)}$ in the FDH scheme, they correspond to coefficients of a perturbative expansion defined as in eq. (2.4), but where $\alpha_{s}(\mu)$ is the $\mathrm{FDH} / \overline{\mathrm{DR}}$ coupling, $\alpha_{s}^{\overline{\mathrm{DR}}}(\mu)$. In the more general $\delta_{R}$ scheme, the expansion parameter would be $\alpha_{s}^{\delta_{R}}(\mu)$.

In this convention, the one-loop relation between $g g \rightarrow g g$ helicity amplitudes is $[22,33]$

$$
\left|\mathcal{M}_{g g \rightarrow g g}^{(1)}\right\rangle_{\delta_{R}}=\left|\mathcal{M}_{g g \rightarrow g g}^{(1)}\right\rangle_{\mathrm{HV}}+\frac{C_{A}}{6}\left(1-\delta_{R}\right)\left|\mathcal{M}_{g g \rightarrow g g}^{(0)}\right\rangle,
$$

which only involves a finite shift. The corresponding relation at two loops also requires shifts of the divergent terms in the infrared decomposition (2.8). We find that

$$
\begin{aligned}
K_{\delta_{R}}= & K_{\mathrm{HV}}-C_{A}\left(\frac{1}{6}+\frac{4}{9} \epsilon\right)\left(1-\delta_{R}\right), \\
\left(H_{g}^{(2)}\right)_{\delta_{R}}= & H_{g}^{(2)}-\frac{C_{A}}{6} b_{0}\left(1-\delta_{R}\right), \\
\left|\mathcal{M}_{g g \rightarrow g g}^{(2) \mathrm{fin}}\right\rangle_{\delta_{R}}= & \left|\mathcal{M}_{g g \rightarrow g g}^{(2) \mathrm{fin}}\right\rangle_{\mathrm{HV}}+ \\
& +\left[C_{A}^{2}\left(-\frac{5}{144} \pi^{2}+\frac{5}{12}\right)+T_{R} N_{f}\left(\frac{8}{27} C_{A}-\frac{1}{2} C_{F}\right)\right]\left(1-\delta_{R}\right)\left|\mathcal{M}_{g g \rightarrow g g}^{(0)}\right\rangle,
\end{aligned}
$$

where $K_{\mathrm{HV}}$ is given in eq. (2.13) and $H_{g}^{(2)}$ (the value in the HV or CDR schemes) is given in eq. (4.38). Because $\boldsymbol{I}^{(1)}(2 \epsilon, \mu ;\{p\})$ contains at most $1 / \epsilon^{2}$ poles, the term proportional to $\epsilon \times\left(1-\delta_{R}\right)$ in $K_{\delta_{R}}$ clearly could be shifted into $\left(H_{g}^{(2)}\right)_{\delta_{R}}$ if desired. However, the assignment we have chosen makes eq. (5.10) simpler. 
Also, the interpretation of $K_{\mathrm{R} . \mathrm{S}}$ as the integral of a splitting function ${ }^{1}[42,60]$ leads to both the $\epsilon^{0}$ and $\epsilon$ terms proportional to $\left(1-\delta_{R}\right)$ in eq. (5.8): The azimuthally-averaged $g \rightarrow g g$ splitting kernel is given in the general $\delta_{R}$ scheme by [60]

$$
\left\langle\hat{P}_{g g}^{\delta_{R}}(z ; \epsilon)\right\rangle=2 C_{A}\left[\frac{z}{1-z}+\frac{1-z}{z}+\left(1+\frac{\epsilon}{1-\epsilon}\left(1-\delta_{R}\right)\right) z(1-z)\right] .
$$

With the identification [60]

$$
-\frac{1}{2} \sum_{b} \int_{0}^{1} d z(z(1-z))^{-\epsilon}\left\langle\hat{P}_{g g}^{\delta_{R}}(z ; \epsilon)\right\rangle=\frac{2 \boldsymbol{T}_{a}^{2}}{\epsilon}+\gamma_{a}+\left(K_{a}-\frac{\pi^{2}}{6} \boldsymbol{T}_{a}^{2}\right) \epsilon,
$$

one sees that the $\delta_{R}$-dependent term of $K \equiv K_{g}$ is

$$
-\frac{C_{A}}{1-\epsilon}\left(1-\delta_{R}\right) \int_{0}^{1} d z(z(1-z))^{1-\epsilon}=-C_{A}\left(\frac{1}{6}+\frac{4}{9} \epsilon\right)\left(1-\delta_{R}\right)
$$

in agreement with eq. (5.8).

One can also present results for the two-loop $g g \rightarrow g g$ amplitudes using the $\delta_{R}$ scheme as the infrared regulator, but switching to the $\overline{\mathrm{MS}}$ coupling constant with the aid of eq. (5.5). For the infrared decomposition (2.8) to hold, assuming that $\boldsymbol{I}^{(1)}(\epsilon)$ is scheme-independent, we find that the quantity $K_{\mathrm{R} . \mathrm{s}}$ must be set to

$$
\tilde{K}_{\delta_{R}}=K_{\mathrm{HV}}+\frac{C_{A}}{2}\left(1-\delta_{R}\right)+\mathcal{O}(\epsilon) .
$$

Thus such a definition of $K$ would be scheme dependent too. Also, in contrast to the simplicity of eq. (5.9), the scheme-dependent part of $\boldsymbol{H}^{(2)}$ will contain logarithms and will no longer be proportional to the identity matrix in color space. Hence we refrain from presenting such a decomposition explicitly.

Finally we discuss conversion from the HV scheme results reported in section 4 to the CDR scheme used in ref. [12]. In the CDR scheme, one usually computes the interference of amplitudes, summed over all external colors and $(2-2 \epsilon)$ polarizations. The generic one-loop/tree interference encountered at NLO is

$$
2 \operatorname{Re} \bar{I}_{\mathrm{RS} .}^{(1,0)} \equiv 2 \operatorname{Re} \sum_{\text {color,hel. }}\left[\left\langle\mathcal{M}_{n}^{(1)} \mid \mathcal{M}_{n}^{(0)}\right\rangle\right]_{\text {R.S. }} .
$$

Inserting the infrared decomposition (2.7) for $\mathcal{M}_{n}^{(1)}$ into eq. (5.15) gives

$$
\bar{I}_{\mathrm{RSS}}^{(1,0)}=2 \operatorname{Re} \sum_{\text {color,hel. }}\left[\left\langle\mathcal{M}_{n}^{(0)}\left|\boldsymbol{I}^{(1)}\right| \mathcal{M}_{n}^{(0)}\right\rangle\right]_{\mathrm{RS.}}+\bar{I}_{\mathrm{RS}}^{(1,0) f i n}
$$

where

$$
\bar{I}_{\text {R.S. }}^{(1,0) \text { fin }}=2 \operatorname{Re} \sum_{\text {color,hel. }}\left[\left\langle\mathcal{M}_{n}^{(1) f i n} \mid \mathcal{M}_{n}^{(0)}\right\rangle\right]_{\text {R.S. }} .
$$

${ }^{1}$ We thank Henry Wong for clarifying this point. 
It is well-established from explicit calculations and general arguments [22, 33, 60] that the finite remainder (5.17) has the same value in the HV and CDR schemes, in the limit $\epsilon \rightarrow 0$. Essentially, the treatment of unobserved partons is the same in both schemes, so the infrared divergences should take the same form, when expressed in terms of the lower-order-in- $\alpha_{s}$ amplitudes.

It is natural to expect the same pattern to hold at two loops. The two-loop/tree interference is

$$
\begin{aligned}
2 \operatorname{Re} \bar{I}_{\mathrm{R.S}}^{(2,0)} & \equiv 2 \operatorname{Re} \sum_{\text {color,hel. }}\left[\left\langle\mathcal{M}_{n}^{(2)} \mid \mathcal{M}_{n}^{(0)}\right\rangle\right]_{\mathrm{RSS}} \\
& =2 \operatorname{Re} \sum_{\text {color,hel. }}\left[\left\langle\mathcal{M}_{n}^{(0)}\left|\boldsymbol{I}^{(2)}\right| \mathcal{M}_{n}^{(0)}\right\rangle+\left\langle\mathcal{M}_{n}^{(1)}\left|\boldsymbol{I}^{(1) \dagger}\right| \mathcal{M}_{n}^{(0)}\right\rangle\right]_{\mathrm{R} . \mathrm{S} .}+\bar{I}_{\mathrm{R} \text {.S. }}^{(2,0) \text { fin }}
\end{aligned}
$$

where

$$
\bar{I}_{\mathrm{RSS}}^{(2,0) \mathrm{fin}}=2 \operatorname{Re} \sum_{\text {color,hel. }}\left[\left\langle\mathcal{M}_{n}^{(2) \mathrm{fin}} \mid \mathcal{M}_{n}^{(0)}\right\rangle\right]_{\mathrm{RS.}} .
$$

Note that $\boldsymbol{I}^{(1)}$ and $\boldsymbol{I}^{(2)}$ are the same operators in the HV scheme as in the CDR scheme.

We have interfered the color-decomposed finite remainders of the two-loop $g g \rightarrow g g$ helicity amplitudes in the HV scheme, as given in section 4, with the tree amplitudes given in eq. (2.22), summing over all external helicities and colors with the help of eq. (2.24). This sum gives precisely the same result as the corresponding quantity (5.20) in CDR scheme, as evaluated in ref. [12], after accounting for the slightly different definition of $\boldsymbol{H}^{(2)}$ that we used in eq. (4.40). We conclude that eq. (5.20) should be the same in the HV or CDR schemes for general two-loop QCD scattering amplitudes.

\section{Two-loop amplitudes in pure $N=1$ super-Yang-Mills theory}

The quarks of QCD are (massless) fermions transforming in the fundamental representation of $S U(N)$. If one replaces the quarks by a gluino, a massless Majorana fermion transforming in the adjoint representation, one obtains a supersymmetric theory, pure $N=1$ super-YangMills theory. The amplitudes for this theory, when it is regularized in a supersymmetry preserving fashion, obey supersymmetry Ward identities [16], and from experience at one loop they are expected to be simpler than the corresponding QCD amplitudes. On the other hand, $S U(N)$ group theory generates linear relations between amplitudes of the two theories, so one can use the two-loop super-Yang-Mills amplitudes to simplify the presentation of the two-loop QCD amplitudes, as we do in appendix A.

In this section we discuss the supersymmetry Ward identities and infrared decomposition for the two-loop amplitudes in pure $N=1$ super-Yang-Mills theory. Then we describe the finite remainder functions for these amplitudes, deferring the most complicated formulas to appendix B.

Here we work in the FDH scheme discussed in section 5, in order that the Ward identities are valid. One set of identities implies that "maximal helicity violating" amplitudes 
vanish for any supersymmetric theory and any number of loops,

$$
\begin{aligned}
& \mathcal{M}_{n}^{\mathrm{SUSY}}\left(g_{1}^{ \pm}, g_{2}^{+}, g_{3}^{+}, \ldots, g_{n}^{+}\right)=0, \\
& \mathcal{M}_{n}^{\mathrm{SUSY}}\left(\tilde{g}_{1}^{-}, \tilde{g}_{2}^{+}, g_{3}^{+}, \ldots, g_{n}^{+}\right)=0,
\end{aligned}
$$

where $g(\tilde{g})$ denotes a gluon (gluino), and the superscripts denote helicities in the alloutgoing convention. In addition to eqs. (6.1) and (6.2), all other super-Yang-Mills $n$-point amplitudes containing either zero or one negative-helicity particle vanish trivially, by using gluino helicity conservation. We have checked that eq. (6.1) is indeed obeyed for the fourpoint amplitude $g g \rightarrow g g$ at two loops in the FDH scheme [23].

Other identities relate the non-vanishing supersymmetric helicity amplitudes for external gluons alone, to amplitudes where some of the gluons are replaced by gluinos. For the four-point amplitudes, all the non-vanishing amplitudes in pure $N=1$ super-Yang-Mills theory can be related to the $g g \rightarrow g g$ amplitudes [33]:

$$
\begin{aligned}
& \mathcal{M}_{4}^{\mathrm{SYM}}\left(\tilde{g}_{1}^{-}, \tilde{g}_{2}^{+}, g_{3}^{-}, g_{4}^{+}\right)=\frac{\langle 23\rangle}{\langle 13\rangle} \mathcal{M}_{4}^{\mathrm{SYM}}\left(g_{1}^{-}, g_{2}^{+}, g_{3}^{-}, g_{4}^{+}\right), \\
& \mathcal{M}_{4}^{\mathrm{SYM}}\left(\tilde{g}_{1}^{-}, \tilde{g}_{2}^{+}, \tilde{g}_{3}^{-}, \tilde{g}_{4}^{+}\right)=\frac{\langle 24\rangle}{\langle 13\rangle} \mathcal{M}_{4}^{\mathrm{SYM}}\left(g_{1}^{-}, g_{2}^{+}, g_{3}^{-}, g_{4}^{+}\right) .
\end{aligned}
$$

These relations are crossing symmetric, when a crossing symmetric definition [63] of the spinor products is used. Thus, to obtain all the $\tilde{g} \tilde{g} \rightarrow g g, \tilde{g} g \rightarrow \tilde{g} g, g g \rightarrow \tilde{g} \tilde{g}$, and $\tilde{g} \tilde{g} \rightarrow \tilde{g} \tilde{g}$ amplitudes from eqs. (6.3) and (6.4), it suffices to give the two independent non-vanishing helicity amplitudes for $g g \rightarrow g g$, namely the --++ and -+-+ configurations.

First we present the infrared decomposition of the pure $N=1$ super-Yang-Mills amplitudes at two loops. The equations in section 2 hold with a few modifications for the super-Yang-Mills case. We use the perturbative expansion (2.4) but in terms of the FDH (or $\overline{\mathrm{DR}}$ ) coupling (5.3). The group-theoretic replacements required to convert the quarks to gluinos are

$$
C_{F} \rightarrow C_{A}, \quad T_{R} N_{f} \rightarrow C_{A} / 2 .
$$

Some of the previous equations, such as eq. (4.38) for $H_{g}^{(2)}$, are given for gauge group $S U(N)$ in terms of $N$ and $N_{f}$, with $T_{R}=1 / 2$, rather than in terms of general Casimir operators. In such equations, to recover the Casimir representation, one should first substitute $N_{f} \rightarrow 2 T_{R} N_{f}$ and $1 / N \rightarrow\left(C_{A}-2 C_{F}\right)$, followed by $N \rightarrow C_{A}$. Then one can apply the substitutions (6.5).

The first two coefficients of the beta function for pure $N=1$ super-Yang-Mills theory are

$$
b_{0}^{\mathrm{SYM}}=\frac{3}{2} C_{A}, \quad b_{1}^{\mathrm{SYM}}=\frac{3}{2} C_{A}^{2},
$$

and $\gamma_{g}$ is similarly modified,

$$
\gamma_{g}^{\mathrm{SYM}}=\frac{3}{2} C_{A} .
$$

The coefficients $K$ and $H_{g}^{(2)}$ are given in the FDH scheme, via eqs. (5.8) and (5.9), by

$$
K_{\mathrm{FDH}}^{\mathrm{SYM}}=\left(3-\frac{\pi^{2}}{6}-\frac{4}{9} \epsilon\right) C_{A},
$$




$$
\left(H_{g}^{(2)}\right)_{\mathrm{FDH}}^{\mathrm{SYM}}=\left(\frac{\zeta_{3}}{2}+\frac{\pi^{2}}{16}-\frac{2}{9}\right) C_{A}^{2}
$$

With these replacements, the one- and two-loop infrared decompositions (2.7) and (2.8) hold in the super-Yang-Mills case. We also color decompose the amplitudes and strip off the helicity phases exactly as in eq. (2.15) for the QCD case. The dependence of the oneand two-loop finite remainders on $\mu$ and $N$ are then extracted as

$$
\begin{aligned}
& M_{\lambda_{1} \lambda_{2} \lambda_{3} \lambda_{4}}^{(1), \text { },[i] \mathrm{fin}}=-b_{0}^{\mathrm{SYM}}\left(\ln \left(s / \mu^{2}\right)-i \pi\right) M_{\lambda_{1} \lambda_{2} \lambda_{3} \lambda_{4}}^{(0),[i]}+N a_{\lambda_{1} \lambda_{2} \lambda_{3} \lambda_{4}}^{\mathrm{SYM},[i]}, \quad i=1,2,3, \\
& M_{\lambda_{1} \lambda_{2} \lambda_{3} \lambda_{4}}^{(1), \text { SYM }[i] \mathrm{fin}}=g_{\lambda_{1} \lambda_{2} \lambda_{3} \lambda_{4}}^{\mathrm{SYM},[i]}, \quad i=7,8,9, \\
& M_{\lambda_{1} \lambda_{2} \lambda_{3} \lambda_{4}}^{(2),[i] \mathrm{fin}}=-\left[\left(b_{0}^{\mathrm{SYM}}\right)^{2}\left(\ln \left(s / \mu^{2}\right)-i \pi\right)^{2}+b_{1}^{\mathrm{SYM}}\left(\ln \left(s / \mu^{2}\right)-i \pi\right)\right] M_{\lambda_{1} \lambda_{2} \lambda_{3} \lambda_{4}}^{(0),[i]}- \\
& -2 b_{0}^{\mathrm{SYM}}\left(\ln \left(s / \mu^{2}\right)-i \pi\right) M_{\lambda_{1} \lambda_{2} \lambda_{3} \lambda_{4}}^{(1), \mathrm{SYM},[i] \mathrm{fin}}+N^{2} A_{\lambda_{1} \lambda_{2} \lambda_{3} \lambda_{4}}^{\mathrm{SYM},[i]}+B_{\lambda_{1} \lambda_{2} \lambda_{3} \lambda_{4}}^{\mathrm{SYM},[i]}, \\
& i=1,2,3 \text {, } \\
& M_{\lambda_{1} \lambda_{2} \lambda_{3} \lambda_{4}}^{(2), \mathrm{irg}}=-2 b_{0}^{\mathrm{SYM}}\left(\ln \left(s / \mu^{2}\right)-i \pi\right) M_{\lambda_{1} \lambda_{2} \lambda_{3} \lambda_{4}}^{(1), \text { SYM },[i] \mathrm{fin}}+N G_{\lambda_{1} \lambda_{2} \lambda_{3} \lambda_{4}}^{\mathrm{SYM},[i]}, \\
& i=7,8,9 \text {. }
\end{aligned}
$$

The one-loop supersymmetric remainder functions are given in terms of the QCD ones,

$$
\begin{aligned}
& a_{\lambda_{1} \lambda_{2} \lambda_{3} \lambda_{4}}^{\mathrm{SYM},[i]}=a_{\lambda_{1} \lambda_{2} \lambda_{3} \lambda_{4}}^{[i]}+c_{\lambda_{1} \lambda_{2} \lambda_{3} \lambda_{4}}^{[i]}+\frac{1}{6} M_{\lambda_{1} \lambda_{2} \lambda_{3} \lambda_{4}}^{(0),[i]}, \quad i=1,2,3, \\
& g_{\lambda_{1} \lambda_{2} \lambda_{3} \lambda_{4}}^{\mathrm{SYM},[i]}=2\left(a_{\lambda_{1} \lambda_{2} \lambda_{3} \lambda_{4}}^{\mathrm{SYM},[1]}+a_{\lambda_{1} \lambda_{2} \lambda_{3} \lambda_{4}}^{\mathrm{SYM},[2]}+a_{\lambda_{1} \lambda_{2} \lambda_{3} \lambda_{4}}^{\mathrm{SYM},[3]}\right), \quad i=7,8,9 .
\end{aligned}
$$

The $M^{(0),[i]}$ correction term in eq. (6.13) is a consequence of using two different schemes FDH for super-Yang-Mills theory vs. HV for QCD. The two-loop analogues of eq. (6.13) are eqs. (A.33), (A.36), (A.37), (A.49), (A.50), (A.54), (A.55), and (A.56) in appendix A. These equations also have correction terms due to the different schemes used, as well as feed-down from the subtracted singular terms, which depend on the fermion representation. The correction terms are more complicated than at one loop, involving $M^{(1),[i] \mathrm{fin}}$ as well as $M^{(0),[i]}$, but still they contain no special functions, only logarithms.

The two-loop supersymmetric remainder functions $A^{\mathrm{SYM}}, B^{\mathrm{SYM}}, G^{\mathrm{SYM}}$, obey the same types of identities as the corresponding QCD functions. The group theory relations are,

$$
\begin{aligned}
& G_{\lambda_{1} \lambda_{2} \lambda_{3} \lambda_{4}}^{\mathrm{SYM},[7]}=2\left(A_{\lambda_{1} \lambda_{2} \lambda_{3} \lambda_{4}}^{\mathrm{SYM},[1]}+A_{\lambda_{1} \lambda_{2} \lambda_{3} \lambda_{4}}^{\mathrm{SYM},[2]}+A_{\lambda_{1} \lambda_{2} \lambda_{3} \lambda_{4}}^{\mathrm{SYM},[3]}\right)-B_{\lambda_{1} \lambda_{2} \lambda_{3} \lambda_{4}}^{\mathrm{SYM},[3]}, \\
& G_{\lambda_{1} \lambda_{2} \lambda_{3} \lambda_{4}}^{\mathrm{SYM},[8]}=2\left(A_{\lambda_{1} \lambda_{2} \lambda_{3} \lambda_{4}}^{\mathrm{SYM},[1]}+A_{\lambda_{1} \lambda_{2} \lambda_{3} \lambda_{4}}^{\mathrm{SYM}, 2]}+A_{\lambda_{1} \lambda_{2} \lambda_{3} \lambda_{4}}^{\mathrm{SYM},[3}\right)-B_{\lambda_{1} \lambda_{2} \lambda_{3} \lambda_{4}}^{\mathrm{SYM},[1]} \\
& G_{\lambda_{1} \lambda_{2} \lambda_{3} \lambda_{4}}^{\mathrm{SYM},[9]}=2\left(A_{\lambda_{1} \lambda_{2} \lambda_{3} \lambda_{4}}^{\mathrm{SYM},[]}+A_{\lambda_{1} \lambda_{2} \lambda_{3} \lambda_{4}}^{\mathrm{SYM},[2]}+A_{\lambda_{1} \lambda_{2} \lambda_{3} \lambda_{4}}^{\mathrm{SYM},[3]}-B_{\lambda_{1} \lambda_{2} \lambda_{3} \lambda_{4}}^{\mathrm{SYM},[2]},\right.
\end{aligned}
$$

and

$$
B_{\lambda_{1} \lambda_{2} \lambda_{3} \lambda_{4}}^{\mathrm{SYM},[3]}=-B_{\lambda_{1} \lambda_{2} \lambda_{3} \lambda_{4}}^{\mathrm{SYM},[1]}-B_{\lambda_{1} \lambda_{2} \lambda_{3} \lambda_{4}}^{\mathrm{SYM},[2]} .
$$

The Bose symmetry relations, which hold only for the --++ helicity configuration, are

$$
X_{--++}^{\mathrm{SYM},[2]}(s, t, u)=X_{--++}^{\mathrm{SYM},[1]}(s, u, t), \quad X \in\{A, B\} .
$$


In appendix $\mathrm{B}$, we give the explicit forms for the independent $N=1$ supersymmetric finite remainder functions appearing in eqs. (6.11) and (6.12). This completes the description of the two-loop four-point amplitudes for $N=1$ super-Yang-Mills theory, and simultaneously of the auxiliary functions required for the QCD amplitudes presented in section 4 .

\section{Conclusions}

In this paper we have presented the two-loop amplitudes for gluon-gluon scattering in QCD, and for all of the $2 \rightarrow 2$ scattering processes in pure $N=1$ super-Yang-Mills theory, including the full dependence on external colors and helicities. We found that there is an additional $1 / \epsilon$ pole term, $\hat{\boldsymbol{H}}^{(2)}$ in eq. (4.41), which has nontrivial color dependence, and which vanishes after interfering it with the tree amplitude and summing over colors. We investigated the dependence of the amplitudes on the flavor of dimensional regularization employed. The QCD results, when summed over all external colors and helicities and converted to CDR scheme, are in complete agreement with the previous results of Glover, Oleari, and Tejeda-Yeomans [12]. We also expressed the one-loop-squared contribution to the NNLO $g g \rightarrow g g$ cross section in terms of one-loop finite remainders. Again the appropriate interference, converted to CDR scheme, is in complete agreement with previous results [31].

Much numerical work still remains in order to implement the two-loop amplitudes of this paper, or those of refs. [11, 12], in a numerical program for NNLO jet production at hadron colliders. When that is accomplished, however, the intrinsic precision on the QCD predictions should reach the few percent level, providing a stringent test of the Standard Model at short distances.

\section{Acknowledgments}

We thank Babis Anastasiou, Adrian Ghinculov, Massimiliano Grazzini and Henry Wong for helpful comments. Z.B. thanks SLAC, and L.D. thanks UCLA, for hospitality while this paper was being completed.

\section{A. Finite remainder functions for QCD}

In this appendix, we present the explicit forms for the independent finite remainder functions for $g g \rightarrow g g$ in QCD, which appear in eqs. (4.46) and (4.47). For the ++++ helicity configuration, the functions are

$$
\begin{aligned}
& A_{++++}^{[1]}=\frac{1}{36}\left(11 \tilde{X}-\frac{y^{2}}{x}-8\right), \\
& A_{++++}^{[3]}=\frac{1}{72}\left(22 \tilde{X}-\frac{1}{x y}-8\right)+\{t \leftrightarrow u\} \\
& B_{++++}^{[1]}=\frac{11}{6}(\tilde{X}-2 \tilde{Y})
\end{aligned}
$$




$$
\begin{aligned}
& C_{++++}^{[1]}=\frac{1}{36}\left(-13 \tilde{X}+2 \frac{y^{2}}{x}+16\right) \\
& C_{++++}^{[3]}=\frac{1}{36}\left(-13 \tilde{X}+2 \frac{y}{x}+10\right)+\{t \leftrightarrow u\} \\
& D_{++++}^{[1]}=-\frac{1}{3}(\tilde{X}-2 \tilde{Y})+\frac{1}{4}, \\
& D_{++++}^{[3]}=-\frac{1}{3} \tilde{X}+\frac{1}{8}+\{t \leftrightarrow u\} \\
& E_{++++}^{[1]}=\frac{1}{36}\left(2 \tilde{X}-\frac{y^{2}}{x}+1\right) \\
& E_{++++}^{[3]}=\frac{1}{72}\left(4 \tilde{X}-\frac{1}{x y}+1\right)+\{t \leftrightarrow u\} \\
& F_{++++}^{[1]}=0, \\
& F_{++++}^{[3]}=0, \\
& H_{++++}^{[7]}=\frac{13}{18} \tilde{X}+\frac{1}{4}+\{t \leftrightarrow u\} \\
& H_{++++}^{[8]}=\frac{1}{18}(13 \tilde{X}-38 \tilde{Y})+\frac{1}{2} \\
& I_{++++}^{[7]}=-\frac{1}{9} \tilde{X}+\{t \leftrightarrow u\} \\
& I_{++++}^{[8]}=-\frac{1}{9}(\tilde{X}-2 \tilde{Y})
\end{aligned}
$$

where $x, y, X, Y, \tilde{X}$ and $\tilde{Y}$ are defined in eqs. (3.17) and (3.18).

For -+++ , the functions are

$$
\begin{aligned}
A_{-+++}^{[1]}= & -\frac{1}{12}\left(15 \frac{x}{y^{2}}-\frac{1+x^{3}}{x y}\right)\left(\tilde{X}^{2}+\pi^{2}\right)+\left(\frac{11}{36} \frac{y^{2}}{x}-\frac{5}{4} \frac{1-x}{y}\right) \tilde{X}- \\
& -\frac{5}{4} \frac{1-x y}{x}, \\
A_{-+++}^{[3]}= & -\frac{1}{24}\left(15 x y-\frac{y^{2}}{x}-\frac{x^{2}}{y}\right)\left((X-Y)^{2}+\pi^{2}\right)+ \\
& +\frac{11}{36} \frac{\tilde{X}}{x y}+\frac{5}{4}(x-y) X+\frac{5}{4} \frac{1-x y}{x}+\{t \leftrightarrow u\}, \\
B_{-+++}^{[1]}= & -\frac{y^{2}+2 x}{2 y^{2}}\left(\tilde{X}^{2}+\pi^{2}\right)-\frac{1+2 x y}{2 x^{2}}\left(\tilde{Y}^{2}+\pi^{2}\right)- \\
& -\frac{1}{2}\left(x^{2}+2 y\right)\left((X-Y)^{2}+\pi^{2}\right)+\frac{11}{6}(\tilde{X}-2 \tilde{Y}), \\
C_{-+++}^{[1]} & \left(\frac{11}{8} \frac{x}{y^{2}}-\frac{5}{16}+\frac{y^{2}}{12 x}\right)\left(\tilde{X}^{2}+\pi^{2}\right)+\frac{11}{8} \frac{1-x y}{x}+ \\
& +\left(\frac{11}{8} \frac{1-x}{y}-\frac{13}{36} \frac{y^{2}}{x}\right) \tilde{X}, \\
C_{-+++}^{[3]} & \frac{x}{48 y}\left(4 x^{2}+x y+30 y^{2}\right)\left((X-Y)^{2}+\pi^{2}\right)-
\end{aligned}
$$




$$
\begin{aligned}
& -\left(\frac{11}{8}(x-y)+\frac{13}{36} \frac{1}{x y}\right) \tilde{X}+\frac{11}{16} \frac{1-x y}{x y}+\{t \leftrightarrow u\}, \\
& D_{-+++}^{[1]}=-\frac{1+x^{2}}{16 y^{2}}\left(\tilde{X}^{2}+\pi^{2}\right)+\frac{6-5 y}{24 y} \tilde{X}+\frac{2}{3} \tilde{Y}+\frac{1-x y}{8 x} \text {, } \\
& D_{-+++}^{[3]}=-\frac{1}{16} y^{2}\left((X-Y)^{2}+\pi^{2}\right)+\frac{1}{24}(6 y-5) \tilde{X}+ \\
& +\frac{1-x y}{16 x y}+\{t \leftrightarrow u\} \\
& E_{-+++}^{[1]}=\frac{y^{2}}{18 x} \tilde{X} \\
& E_{-+++}^{[3]}=\frac{1}{18 x y} \tilde{X}+\{t \leftrightarrow u\}, \\
& F_{-+++}^{[1]}=0 \text {, } \\
& F_{-+++}^{[3]}=0 \text {, } \\
& H_{-+++}^{[7]}=\frac{1}{12}\left(2 \frac{y^{2}}{x}+\frac{3 x(1-2 x)}{y^{2}}\right)\left(\tilde{X}^{2}+\pi^{2}\right)+\left(\frac{11}{9} \frac{x^{2}}{y}-\frac{1}{2}\right) \tilde{X}+ \\
& +\frac{1}{24}\left(-4 \frac{y^{2}}{x}+3 x y\right)\left((X-Y)^{2}+\pi^{2}\right)+\{t \leftrightarrow u\}, \\
& H_{-+++}^{[8]}=\frac{1}{12}\left(2 \frac{y^{2}}{x}-3 \frac{2 y^{2}-x}{y^{2}}\right)\left(\tilde{X}^{2}+\pi^{2}\right)+\frac{1}{12}\left(2 \frac{x^{2}}{y}-3 \frac{2-y}{x^{2}}\right)\left(\tilde{Y}^{2}+\pi^{2}\right)- \\
& -\frac{1}{12}\left(2 \frac{x^{2}}{y}-15 x y-8 y^{2}-2 \frac{y^{3}}{x}\right)\left((X-Y)^{2}+\pi^{2}\right)+ \\
& +\frac{1}{18}\left(22 \frac{x^{2}}{y}-9\right) \tilde{X}+\frac{1}{9}\left(11 \frac{y^{2}}{x}-30\right) \tilde{Y} \text {, } \\
& I_{-+++}^{[7]}=-\frac{x^{2}}{9 y} \tilde{X}+\{t \leftrightarrow u\} \text {, } \\
& I_{-+++}^{[8]}=-\frac{1}{9}\left(\frac{x^{2}}{y} \tilde{X}+\left(\frac{y^{2}}{x}-3\right) \tilde{Y}\right) \text {. }
\end{aligned}
$$

For --++ , the functions are

$$
\begin{aligned}
& A_{--++}^{[1]}=\left(x^{2}+6 y^{2}-3 x y\right)[ \operatorname{Li}_{4}\left(-\frac{x}{y}\right)-\mathrm{Li}_{4}(-x)-\mathrm{Li}_{4}(-y)+ \\
&+\tilde{X}\left(\operatorname{Li}_{3}(-x)+\mathrm{Li}_{3}(-y)\right)- \\
&-\frac{\pi^{2}}{6} \mathrm{Li}_{2}(-x)+\frac{1}{12} \tilde{X}^{4}+\frac{1}{6}\left(\tilde{X}^{2}+\pi^{2}\right) \tilde{X} Y+ \\
&\left.+\frac{1}{24} Y^{2}\left(6 X^{2}-4 X Y+Y^{2}+2 \pi^{2}\right)-\frac{17}{720} \pi^{4}\right]+ \\
&+y \frac{y^{2}-3 x y+6 x^{2}}{x}\left(\frac{1}{8} \tilde{X}^{4}+\frac{\pi^{2}}{6} \tilde{X}^{2}+\frac{\pi^{4}}{80}\right)+\frac{\zeta_{3}}{2 x} \tilde{X}+ \\
&+\frac{1}{6} \frac{11+60 x y}{x}\left[\operatorname{Li}_{3}(-x)-\tilde{X} \operatorname{Li}_{2}(-x)+\frac{1}{3} \tilde{X}^{3}-\frac{1}{2}\left(\tilde{X}^{2}+\pi^{2}\right) Y+\right.
\end{aligned}
$$




$$
\begin{aligned}
& \left.+\frac{17}{24} \pi^{2} \tilde{X}-\frac{19}{6} \zeta_{3}\right]-\frac{5}{12} y\left(4 \tilde{X}^{3}+9 \pi^{2} \tilde{X}-52 \zeta_{3}\right)- \\
& -\frac{5}{4}\left(\frac{x^{2}}{y^{2}}-\frac{4}{y}+1\right)\left(\tilde{X}^{2}+\pi^{2}\right)-\frac{\pi^{2}}{36 x}(53+15 y(3 x-y))- \\
& -\left[\frac{5}{2}\left(\frac{x}{y}-2 \frac{y}{x}\right)+\frac{67}{54 x}\right] \tilde{X}-\frac{x}{36}-\frac{25}{18}+\frac{11093}{648 x}, \\
& A_{--++}^{[3]}=-x^{2} \frac{3-2 x y}{24 x y}(X-Y)^{4}-\frac{\pi^{2}}{12} \frac{(1-x y)^{2}}{x y}(X-Y)^{2}+\frac{\zeta_{3}}{2 x y} \tilde{X}-\frac{\pi^{4}}{160 x y}- \\
& -\frac{11}{6 x y}\left(\operatorname{Li}_{3}(-x)-X \operatorname{Li}_{2}(-x)-\frac{1}{3} X^{3}-\frac{7}{8} \pi^{2} X+\frac{13 \zeta_{3}}{12}-i \frac{\pi^{3}}{24}\right)+ \\
& +\left(\frac{11}{12} \frac{x^{3}}{y}-\frac{2}{9}(x-y)\right)\left((X-Y)^{2} X-\frac{\pi^{2}}{3} Y\right)- \\
& -\frac{11}{6} i \pi \frac{(1-x y)^{2}}{y}\left((X-Y)^{2}+\pi^{2}\right)-\frac{\pi^{2}}{27}\left(16 x^{2}-17 x y+58\right) X+ \\
& +\frac{11}{36}\left(\frac{11}{y}-3(x-y)\right)\left(\tilde{X}^{2}+\pi^{2}\right)+\frac{1}{24}(41-30 x y)\left((X-Y)^{2}+\pi^{2}\right)- \\
& -\pi^{2} \frac{1+41(1-x)}{36 y}+\left(\frac{101}{27 y}+5 x+\frac{19}{12}+\frac{337}{54 x}\right) \tilde{X}+\frac{5}{4}-\frac{11093}{648 x}+ \\
& +\{t \leftrightarrow u\} \\
& B_{--++}^{[1]}=B_{--++}^{\mathrm{SYM},[1]}-H_{--++}^{[7]}+2 H_{--++}^{[8]}-H_{--++}^{[9]}+I_{--++}^{[7]}-2 I_{--++}^{[8]}+I_{--++}^{[9]}- \\
& -\frac{1}{3}(x-y)\left(2 \frac{x^{2}}{y}-3\right) \tilde{X}^{3}-\frac{4}{3}\left(2 \frac{x^{2}}{y}-3 x y-\frac{y^{3}}{x}\right) \tilde{X}^{2} \tilde{Y}+ \\
& +\frac{1}{3}\left(8 \frac{x^{2}}{y}+x-20 x y+3 y+10 \frac{y^{2}}{x}\right) \tilde{X} \tilde{Y}^{2}-\frac{2}{3}(x-y)\left(2 \frac{y^{2}}{x}-3\right) \tilde{Y}^{3}+ \\
& +\frac{\pi^{2}}{3}(5-4 x y)(\tilde{X}-2 \tilde{Y})-\frac{2}{9}\left(13 \frac{x^{2}}{y}+x-19\right) \tilde{X}^{2}+ \\
& +\frac{4}{9}\left(13 \frac{y^{2}}{x}+y-19\right) \tilde{Y}^{2}+\frac{2}{9}\left(\frac{26}{y}-18(x-y)-\frac{13}{x}\right) \tilde{X} \tilde{Y}+ \\
& +\frac{4}{3}(\tilde{X}-2 \tilde{Y}) \\
& C_{--++}^{[1]}=\left(2 x^{2}-6 x y+3 y^{2}\right)\left[\operatorname{Li}_{4}(-x)+\operatorname{Li}_{4}(-y)-\operatorname{Li}_{4}\left(-\frac{x}{y}\right)-\tilde{X} \operatorname{Li}_{3}(-x)-\right. \\
& -\tilde{X} \operatorname{Li}_{3}(-y)+\frac{\pi^{2}}{6} \operatorname{Li}_{2}(-x)+\frac{1}{24} \tilde{X}^{4}- \\
& -\frac{1}{4} X^{2}\left(\tilde{Y}^{2}+\pi^{2}\right)-\frac{1}{6} X Y\left(X^{2}-Y^{2}\right)-\frac{1}{24} Y^{4}+ \\
& \left.+\frac{\pi^{2}}{12}\left(2 \tilde{X}^{2}+4 X Y-Y^{2}\right)+\frac{13}{360} \pi^{4}\right]- \\
& -\frac{1}{6}\left(\frac{2}{x}-3(9 x-13 y)\right)\left[\operatorname{Li}_{3}(-x)-\tilde{X} \operatorname{Li}_{2}(-x)+\frac{1}{3} \tilde{X}^{3}-\frac{1}{2}\left(\tilde{X}^{2}+\pi^{2}\right) Y+\right.
\end{aligned}
$$




$$
\begin{aligned}
& \left.+\frac{17}{24} \pi^{2} \tilde{X}-\frac{\zeta_{3}}{6}\right]- \\
& -\frac{1}{12}(9 x-13 y)\left(\tilde{X}^{3}+\frac{9}{4} \pi^{2} \tilde{X}+5 \zeta_{3}\right)+ \\
& +\frac{1}{8}\left(\frac{11}{y^{2}}+4 \frac{x}{y}+26\right)\left(\tilde{X}^{2}+\pi^{2}\right)-\frac{\pi^{2}}{216}\left(38 \frac{y^{2}}{x}-\frac{85}{x}-358 y-101\right)+ \\
& +\frac{1}{108}\left(297 \frac{x+2 y}{y}+\frac{137}{x}\right) \tilde{X}-\frac{y}{18}+\frac{115}{72}-\frac{4849}{1296 x}, \\
& C_{--++}^{[3]}=-\frac{1}{48} x^{2}\left((X-Y)^{2}+\pi^{2}\right)\left(3(X-Y)^{2}-\pi^{2}\right)+ \\
& +\frac{1}{3 x y}\left[\operatorname{Li}_{3}(-x)-X \operatorname{Li}_{2}(-x)-\frac{1}{3} X^{3}-i \frac{\pi}{2} X(X-Y)-\frac{3}{8} \pi^{2} X-\right. \\
& \left.-\frac{5}{12} \zeta_{3}-i \frac{\pi^{3}}{8}\right]-\frac{1}{12}\left(2 \frac{y^{3}}{x}+\frac{x^{2}}{y}(11 y-4 x)\right) \tilde{X}^{2} \tilde{Y}- \\
& -\frac{1}{36}\left(6 \frac{x^{3}}{y}-11\left(2 x^{2}+y^{2}\right)\right) \tilde{X}\left(\tilde{X}^{2}+\pi^{2}\right)+ \\
& +\frac{\pi^{2}}{18}\left(2 \frac{y^{2}}{x}-1+4 x\right) \tilde{X}-\frac{1}{36}\left(\frac{44}{y}-39(x-y)\right)\left(\tilde{X}^{2}+\pi^{2}\right)- \\
& -\frac{1}{24}\left(37 x^{2}+4 x y\right)\left((X-Y)^{2}+\pi^{2}\right)-\pi^{2} \frac{13+204(1-x y)}{432 x y}- \\
& -\frac{1}{108}\left(-\frac{56}{x y}+\frac{9}{x}\left(55 x^{2}-2 x y+9 y^{2}\right)\right) \tilde{X}-\frac{11}{8}+\frac{4849}{1296 x}+ \\
& +\{t \leftrightarrow u\} \\
& D_{--++}^{[1]}=-A_{--++}^{\mathrm{SYM},[1]}+A_{--++}^{[1]}+C_{--++}^{[1]}+E_{--++}^{[1]}+\frac{1}{3}\left(\frac{x^{2}}{y}+y^{2}\right) \tilde{X}^{3}+ \\
& +\frac{2}{3}\left(\frac{x^{3}}{y}-\frac{1}{y}+\frac{y^{3}}{x}\right) \tilde{X}^{2} \tilde{Y}-\frac{1}{3}\left(5 \frac{y}{x}-1+5 x y+4 \frac{x}{y}\right) \tilde{X} \tilde{Y}^{2}+ \\
& +\frac{\pi^{2}}{3}(2-x y)(\tilde{X}-2 \tilde{Y})-\frac{2}{3}\left(\frac{y^{2}}{x}+x^{2}\right) \tilde{Y}^{3}- \\
& -\frac{1}{18}\left(\frac{11}{x}+3(x-y)\right)\left(\tilde{X}^{2}+4 \pi^{2}\right)+ \\
& +\frac{1}{18}\left(\frac{33}{x}+3(x-y)+\frac{22}{y}\right)\left(\tilde{Y}^{2}+\pi^{2}\right)- \\
& -\frac{1}{18}\left(-\frac{11}{x}-9(x-y)+\frac{22}{y}\right)\left((X-Y)^{2}+\pi^{2}\right)+\frac{5}{144 x} \pi^{2}+ \\
& +\frac{1}{3}(\tilde{X}-2 \tilde{Y})-\frac{17}{54 x}, \\
& D_{--++}^{[3]}=-A_{--++}^{\mathrm{SYM},[3]}+A_{--++}^{[3]}+C_{--++}^{[3]}+E_{--++}^{[3]}+ \\
& +\left[\frac{1}{3}\left(\frac{x^{2}}{y}+y^{2}\right) \tilde{X}^{3}+\frac{1}{3}\left(\frac{1}{x y}+x-y+\frac{y^{3}}{x}\right) \tilde{X}^{2} \tilde{Y}+\frac{\pi^{2}}{3}(2-x y) \tilde{X}-\right.
\end{aligned}
$$




$$
\begin{aligned}
& -\frac{1}{9}\left(\frac{11}{y}-3(x-y)\right)\left(\tilde{X}^{2}+\pi^{2}\right)-\frac{11}{9 y} \tilde{X} \tilde{Y}+\frac{19}{16 y} \pi^{2}+ \\
& \left.+\frac{1}{3} \tilde{X}+\frac{17}{54 y}+\{t \leftrightarrow u\}\right] \\
& E_{--++}^{[1]}=-\frac{\pi^{2}}{108 x}-\frac{1}{72}(x-y), \\
& E_{--++}^{[3]}=-\frac{1}{12}\left(x^{2}+y^{2}\right) \tilde{X}\left((X-Y)^{2}+\pi^{2}\right)+\frac{1}{18}\left(2 \frac{x^{2}}{y}+x+5 y\right) \tilde{X}^{2}- \\
& -\frac{\pi^{2}}{216 x y}-\frac{1}{6} \tilde{X}+\{t \leftrightarrow u\} \\
& F_{--++}^{[1]}=\frac{1}{18}\left[\frac{1}{x}\left(\tilde{X}^{2}+4 \pi^{2}\right)+\frac{2-y}{x y}\left(\tilde{Y}^{2}+\pi^{2}\right)+\right. \\
& \left.+\frac{2 x-y}{x y}\left((X-Y)^{2}+\pi^{2}\right)\right] \\
& F_{--++}^{[3]}=\frac{1}{9}\left(\frac{1}{y}\left(\tilde{X}^{2}+\pi^{2}\right)+\frac{X Y}{x}-i \pi \frac{\tilde{X}}{x y}\right)+\{t \leftrightarrow u\} \\
& H_{--++}^{[7]}=\frac{4}{3}(2-x y)\left(\operatorname{Li}_{3}(-x)-\tilde{X} \operatorname{Li}_{2}(-x)+\frac{1}{6} \tilde{X}^{3}-\frac{3}{4} \tilde{X}^{2} \tilde{Y}+i \frac{\pi}{2}\left(\tilde{X}^{2}+\pi^{2}\right)\right)+ \\
& +\frac{1}{36 y} \tilde{X}\left(8 \tilde{X}^{2}+29 \pi^{2}\right)+\frac{1}{6}\left(2 \frac{x^{3}}{y}-6(x-y)-4 \frac{y^{3}}{x}\right) \tilde{X}^{2} \tilde{Y}- \\
& -\frac{1}{18}\left[\left(18 \frac{x}{y^{2}}-3 \frac{x}{y}+8+11 \frac{y}{x}\right)\left(\tilde{X}^{2}+\pi^{2}\right)+\right. \\
& +2\left(\frac{22}{y}-3(x-y)\right)\left(\tilde{X}^{2}+\pi^{2}\right)- \\
& \left.-\left(11 \frac{x^{3}}{y}+8 x^{2}+6 x y\right)\left((X-Y)^{2}+\pi^{2}\right)+\frac{11}{2} \frac{\pi^{2}}{x y}\right]- \\
& -\frac{2}{27} \frac{23+9 x y}{y} \tilde{X}+\frac{1}{4}+\{t \leftrightarrow u\} \\
& H_{--++}^{[8]}=2\left(x^{2}+y^{2}\right)\left[3\left(\operatorname{Li}_{4}(-x)+\operatorname{Li}_{4}(-y)\right)-2 \tilde{X} \operatorname{Li}_{3}(-x)-2 \tilde{Y} \operatorname{Li}_{3}(-y)+\right. \\
& +\frac{1}{2}\left(\tilde{X}^{2}-\tilde{Y}^{2}\right) \operatorname{Li}_{2}(-x)+\frac{1}{6} X^{3} \tilde{Y}-\frac{1}{4}\left(X^{2}+\frac{\pi^{2}}{6}\right) Y^{2}- \\
& \left.-\frac{1}{3}\left(X-i \frac{\pi}{2}\right) \tilde{Y}^{3}-\frac{\pi^{2}}{8}\left(X^{2}+6 X Y-4 \tilde{Y}^{2}\right)+\frac{7}{60} \pi^{4}\right]+ \\
& +4 x(x-3 y)\left[\operatorname{Li}_{4}(-x)+\operatorname{Li}_{4}(-y)+\operatorname{Li}_{4}\left(-\frac{x}{y}\right)-\tilde{Y}\left(\operatorname{Li}_{3}(-x)+\operatorname{Li}_{3}(-y)\right)-\right. \\
& -\frac{\pi^{2}}{6} \operatorname{Li}_{2}(-x)+\frac{1}{12} Y^{4}-\frac{1}{3}\left(X-i \frac{\pi}{2}\right) Y^{3}-
\end{aligned}
$$




$$
\begin{aligned}
& \left.-i \frac{\pi}{2} X Y^{2}+i \frac{\pi^{3}}{6} Y-\frac{\pi^{4}}{24}\right]+ \\
& +\frac{1}{3}\left(2 \frac{x^{3}}{y}-x^{2}+14 y^{2}\right)\left[\operatorname{Li}_{3}(-x)+\zeta_{3}-\tilde{X} \operatorname{Li}_{2}(-x)+i \frac{\pi}{2} X^{2}-\right. \\
& \left.-\frac{11}{8} \pi^{2} X-\frac{3}{8} i \pi^{3}\right]+ \\
& +\frac{1}{3}\left(4 \frac{x^{3}}{y}+17 x^{2}-30 x-2 y^{2}\right)\left[\operatorname{Li}_{3}(-y)-\zeta_{3}-\tilde{Y} \operatorname{Li}_{2}(-y)+i \frac{\pi}{2} Y^{2}-\right. \\
& \left.-\frac{1}{2} \tilde{X} \tilde{Y}(X-Y)-\frac{\pi^{2}}{2}(X-i \pi)\right]+ \\
& +\frac{2}{9}\left(\frac{x^{2}}{y}+y^{2}\right) \tilde{X}^{3}+\frac{1}{12}\left(8 \frac{y^{2}}{x}-15 y^{2}-48 x+27 x^{2}\right) \tilde{X}^{2} \tilde{Y}- \\
& -\frac{1}{6}\left(14 \frac{y^{2}}{x}-7 y^{2}-36 x+34 x^{2}\right) \tilde{X} \tilde{Y}^{2}+\frac{1}{36}\left(44 \frac{y^{2}}{x}-5 y^{2}-48 x+35 x^{2}\right) \tilde{Y}^{3}+ \\
& +\frac{\pi^{2}}{72}\left(182 y^{2}+96 x-42 y+21 x^{2}\right) \tilde{X}- \\
& -\frac{\pi^{2}}{36}\left(29 \frac{y^{3}}{x}-2 y^{2}-63 x+155 x^{2}-\frac{48}{y}\right) \tilde{Y}-2 \zeta_{3}(x-y)+ \\
& +\frac{1}{36}\left(\frac{9}{y^{2}}-\frac{44}{y}+3(1-3 x)(1-7 x)\right)\left(\tilde{X}^{2}+\pi^{2}\right)+ \\
& +\frac{1}{36}\left(\frac{36}{x^{2}}-\frac{212}{x}+3\left(y^{2}+4 x y+24 x^{2}\right)\right)\left(\tilde{Y}^{2}+\pi^{2}\right)- \\
& -\frac{1}{18}\left(\frac{44}{y}-\frac{22}{x}-3\left(2 x^{2}+52 x y+29 y^{2}\right)\right) \tilde{X} \tilde{Y}+ \\
& +\frac{\pi^{2}}{36}\left(\frac{44}{y}+\frac{176}{x}-55 x^{2}+8 x y-144 y\right)-\frac{1}{54}\left(\frac{83}{y}+45 x-9\right) \tilde{X}- \\
& -\frac{1}{54}\left(\frac{92}{x}+45 y-144\right) \tilde{Y}, \\
& I_{--++}^{[7]}=\frac{1}{9 y} \tilde{X}(X-Y)+\{t \leftrightarrow u\}, \\
& I_{--++}^{[8]}=\frac{1}{18}\left[-3\left(x^{2}+y^{2}\right)\left((X-Y)^{2}+\pi^{2}\right) \tilde{Y}-\left(\frac{y^{2}}{x}+4 x-y\right)\left(\tilde{X}^{2}+\pi^{2}\right)+\right. \\
& +\frac{4}{y}\left(\tilde{X}^{2}+\frac{\pi^{2}}{2}\right)+\left(2 \frac{x^{2}}{y}+7 x-y+\frac{7}{x}\right) \tilde{Y}^{2}+ \\
& \left.+\left(\frac{y^{2}}{x}-3 y-2 \frac{x^{2}}{y}\right)\left((X-Y)^{2}+\pi^{2}\right)-6 \tilde{Y}\right] \text {. }
\end{aligned}
$$

For -+-+ , the functions are

$$
A_{-+-+}^{[1]}=-\frac{1}{24}\left(1+x^{2}\right)\left(\frac{3}{x}-\frac{2}{y^{2}}\right) \tilde{X}^{4}-\frac{\pi^{2}}{6} \frac{(1-x y)^{2}}{x y^{2}} \tilde{X}^{2}+\frac{1}{2} \zeta_{3} \frac{y^{2}}{x} \tilde{X}-\frac{\pi^{4}}{80} \frac{y^{2}}{x}+
$$




$$
\begin{aligned}
& +\frac{11}{6} \frac{y^{2}}{x}\left[\operatorname{Li}_{3}(-x)-\tilde{X} \operatorname{Li}_{2}(-x)-\frac{1}{2}\left(\tilde{X}^{2}+\pi^{2}\right) \tilde{Y}+\frac{1}{3} X^{3}+\frac{3}{2} i \pi X^{2}-\right. \\
& \left.-\frac{31}{24} \pi^{2} X+\frac{3}{8} i \pi^{3}-\frac{19}{6} \zeta_{3}\right]+ \\
& +\left(\frac{11}{12} \frac{x^{3}}{y^{2}}+\frac{2}{9} \frac{1-x}{y}\right)\left(\tilde{X}^{2}+\pi^{2}\right) \tilde{X}+\frac{\pi^{2}}{18}\left(\frac{6}{y}-3 x+8 y\right) \tilde{X}- \\
& -\frac{1}{36}\left(90 \frac{x^{3}}{y^{2}}-114 \frac{x}{y}-31 y\right)\left(\tilde{X}^{2}+\pi^{2}\right)-\frac{\pi^{2}}{36}\left(66 \frac{x^{2}}{y}-17 x+4+\frac{38}{x}\right)+ \\
& +\left(\frac{101}{27} y+5 \frac{x}{y}+\frac{19}{12}+\frac{337}{54} \frac{y}{x}\right)(X-Y)+ \\
& +\left(\frac{337}{54} \frac{y}{x}-\frac{5}{y}-\frac{41}{12}+\frac{101}{27} y\right) \tilde{Y}+\frac{5}{2}+\frac{11093}{648} \frac{y^{2}}{x}, \\
& A_{-+-+}^{[2]}=-\frac{1+6 x^{2}-3 x}{y^{2}}\left[\operatorname{Li}_{4}\left(-\frac{x}{y}\right)+\operatorname{Li}_{4}(-x)-\operatorname{Li}_{4}(-y)-\tilde{Y} \operatorname{Li}_{3}(-x)+\right. \\
& \left.+\frac{\pi^{2}}{6} \operatorname{Li}_{2}(-x)-\frac{1}{12} \tilde{Y}^{4}-\frac{1}{6} Y\left(\tilde{X} Y^{2}-\pi^{2}(Y-i \pi)\right)+\zeta_{3} \tilde{Y}+\frac{67}{720} \pi^{4}\right]+ \\
& +\frac{x}{y^{2}}\left(x^{2}-3 x+6\right)\left(\frac{1}{8} \tilde{Y}^{4}+\frac{\pi^{2}}{6} \tilde{Y}^{2}+\frac{\pi^{4}}{80}\right)+\frac{y}{2} \zeta_{3} \tilde{Y}+ \\
& +\frac{1}{6}\left(11+60 \frac{x}{y^{2}}\right) y\left[\operatorname{Li}_{3}(-y)-\tilde{Y} \operatorname{Li}_{2}(-y)-\frac{1}{3} \tilde{Y}^{3}-\right. \\
& \left.-\frac{1}{2}\left(\tilde{Y}^{2}+\pi^{2}\right)(X-\tilde{Y})-\frac{17}{24} \pi^{2} \tilde{Y}-\frac{19}{6} \zeta_{3}\right]- \\
& -\frac{5}{12} \frac{x}{y}\left(4 \tilde{Y}^{3}-9 \pi^{2} \tilde{Y}-52 \zeta_{3}\right)+\frac{11}{72} y\left(8 \tilde{Y}^{2}+13 \pi^{2}\right) \tilde{Y}- \\
& -\frac{5}{4}\left(\frac{1}{x^{2}}-4 \frac{y}{x}+1+\frac{121}{45} y\right)\left(\tilde{Y}^{2}+\pi^{2}\right)+\frac{\pi^{2}}{36}\left(\frac{60}{y}-75 x+8 y\right)+ \\
& +\left[\frac{5}{2}\left(\frac{1}{x}-2 x\right)-\frac{236}{27} y\right] \tilde{Y}-\frac{1}{36 y}-\frac{25}{18}+\frac{11093}{648} y, \\
& A_{-+-+}^{[3]}=-\frac{x^{2}+6-3 x}{y^{2}}\left[\operatorname{Li}_{4}\left(-\frac{x}{y}\right)-\operatorname{Li}_{4}(-x)-\operatorname{Li}_{4}(-y)+\right. \\
& +(X-Y)\left(\operatorname{Li}_{3}(-x)-\zeta_{3}\right)-\frac{\pi^{2}}{6}\left(\operatorname{Li}_{2}(-x)+\frac{1}{2} Y^{2}\right)+ \\
& \left.+\frac{11}{240} \pi^{4}-\frac{1}{12}\left((X-Y)^{4}-\left(2 X^{2}-3 X Y+4 \pi^{2}\right) X Y\right)\right]+ \\
& +\frac{1-3 x+6 x^{2}}{x y^{2}}\left(\frac{1}{8}(X-Y)^{4}+\frac{\pi^{2}}{6}(X-Y)^{2}+\frac{\pi^{4}}{80}\right)+\frac{y}{2 x} \zeta_{3}(\tilde{X}+\tilde{Y})- \\
& -\frac{1}{6}\left(11 \frac{y}{x}+\frac{60}{y}\right)\left[\operatorname{Li}_{3}(-x)+\operatorname{Li}_{3}(-y)-(X-Y) \operatorname{Li}_{2}(-x)-\right.
\end{aligned}
$$




$$
\begin{aligned}
& -\frac{1}{6}(2 X-Y)\left((X+Y)^{2}-6 Y^{2}\right)- \\
& -i \pi\left((X-Y)^{2}+\pi^{2}\right)- \\
& \left.-\frac{\pi^{2}}{24}(17 X+25 Y+2 i \pi)+\frac{13}{6} \zeta_{3}\right]- \\
& -\frac{5}{12 y}\left[4(\tilde{X}+5 \tilde{Y})\left((X-Y)^{2}+\pi^{2}\right)+\pi^{2}(5 \tilde{X}-3 \tilde{Y})-52 \zeta_{3}\right]- \\
& -\frac{5}{4}\left(x^{2}-4 y+1\right)\left((X-Y)^{2}+\pi^{2}\right)- \\
& -\frac{121}{36} \frac{y}{x}\left(\tilde{Y}^{2}+\pi^{2}\right)+\frac{\pi^{2}}{36}\left(83 \frac{y}{x}+15-\frac{60}{y}\right)-\frac{5}{2}\left(x-\frac{2}{x}\right)(X-Y)- \\
& -\frac{y}{54 x}(67 \tilde{X}+472 \tilde{Y})-\frac{x}{36 y}-\frac{25}{18}+\frac{11093}{648} \frac{y}{x}, \\
& B_{-+-+}^{[1]}=B_{-+-+}^{\mathrm{SYM},[1]}-H_{-+-+}^{[7]}+2 H_{-+-+}^{[8]}-H_{-+-+}^{[9]}+I_{-+-+}^{[7]}-2 I_{-+-+}^{[8]}+I_{-+-+}^{[9]}+ \\
& +\frac{1}{3}(1-x)\left(2 \frac{x^{2}}{y^{2}}-\frac{3}{y}\right) \tilde{X}^{3}-\frac{2}{3}\left(\frac{4}{y^{2}}+3 \frac{x^{2}}{y}-\frac{1}{x y}+\frac{y^{2}}{x}\right) \tilde{X}^{2} \tilde{Y}- \\
& -\frac{2}{3}(4-y) \frac{y}{x} \tilde{X} \tilde{Y}^{2}-\frac{4}{3} \frac{y^{2}}{x} \tilde{Y}^{3}-\frac{\pi^{2}}{3}\left(4 \frac{x}{y^{2}}-5\right)(\tilde{X}-2 \tilde{Y})- \\
& -\frac{2}{9}\left(12 \frac{x^{2}}{y}-x-19\right) \tilde{X}^{2}+\frac{52}{9} \frac{y^{2}}{x} \tilde{Y}^{2}+\frac{2}{9}\left(\frac{24}{y}-1+39 y-\frac{13}{x}\right) \tilde{X} \tilde{Y}+ \\
& +\frac{4}{3}(\tilde{X}-2 \tilde{Y}) \\
& B_{-+-+}^{[2]}=B_{-+-+}^{\mathrm{SYM},[2]}-H_{-+-+}^{[7]}-H_{-+-+}^{[8]}+2 H_{-+-+}^{[9]}+I_{-+-+}^{[7]}+I_{-+-+}^{[8]}-2 I_{-+-+}^{[9]}- \\
& -\frac{2}{3}(1-x)\left(2 \frac{x^{2}}{y^{2}}-\frac{3}{y}\right) \tilde{X}^{3}-\frac{1}{3}\left(4 \frac{x}{y^{2}}+5(x-y)-8 \frac{y^{2}}{x}\right) \tilde{X}^{2} \tilde{Y}+ \\
& +\frac{4}{3}(1-y) \frac{y}{x} \tilde{X} \tilde{Y}^{2}+\frac{2}{3} \frac{y^{2}}{x} \tilde{Y}^{3}+\frac{\pi^{2}}{3}\left(4 \frac{x}{y^{2}}-5\right)(2 \tilde{X}-\tilde{Y})+ \\
& +\frac{4}{9}\left(12 \frac{x^{2}}{y}-x-19\right) \tilde{X}^{2}-\frac{26}{9} \frac{y^{2}}{x} \tilde{Y}^{2}-\frac{2}{9}\left(\frac{12}{y}-20+39 y-\frac{26}{x}\right) \tilde{X} \tilde{Y}- \\
& -\frac{4}{3}(2 \tilde{X}-\tilde{Y}) \text {, } \\
& C_{-+-+}^{[1]}=-\frac{1}{48} \frac{1+x^{2}}{y^{2}}\left(3 \tilde{X}^{4}+2 \pi^{2} \tilde{X}^{2}-\pi^{4}\right)- \\
& -\frac{y^{2}}{3 x}\left[\operatorname{Li}_{3}(-x)-\tilde{X} \operatorname{Li}_{2}(-x)+\frac{1}{3} \tilde{X}^{3}-\frac{1}{2}\left(\tilde{X}^{2}+\pi^{2}\right) Y+\frac{17}{24} \pi^{2} \tilde{X}-\frac{1}{6} \zeta_{3}\right]- \\
& -\frac{1}{36}\left(39 \frac{x^{3}}{y^{2}}+11 \frac{1-x(1-3 x)}{y}\right) \tilde{X}\left(\tilde{X}^{2}+\pi^{2}\right)-\frac{\pi^{2}}{18}\left(\frac{6}{y}-2 x+1\right) \tilde{X}+ \\
& +\frac{1}{72}\left(-198 \frac{x^{2}}{y^{2}}-42 \frac{x}{y}+33 x-55 y\right)\left(\tilde{X}^{2}+\pi^{2}\right)-
\end{aligned}
$$




$$
\begin{aligned}
& -\frac{\pi^{2}}{216}\left(468 \frac{x}{y}+17 x-200 y-\frac{47}{x}\right)-\frac{1}{108}\left(594 \frac{x}{y}-43 x+13 y-\frac{137}{x}\right) \tilde{X}- \\
& -\frac{11}{4}-\frac{4849}{1296} \frac{y^{2}}{x} \\
& C_{-+-+}^{[2]}=\frac{2-6 x+3 x^{2}}{y^{2}}\left[\operatorname{Li}_{4}\left(-\frac{x}{y}\right)+\operatorname{Li}_{4}(-x)-\operatorname{Li}_{4}(-y)-\tilde{Y}\left(\operatorname{Li}_{3}(-x)-\zeta_{3}\right)+\right. \\
& \left.+\frac{\pi^{2}}{6} \operatorname{Li}_{2}(-x)-\frac{1}{24}\left(4 X Y-Y^{2}-2 \pi^{2}\right) Y^{2}-\frac{7}{360} \pi^{4}\right]- \\
& -\frac{1}{6}\left(2 y-3 \frac{9-13 x}{y}\right)\left[\operatorname{Li}_{3}(-y)-\tilde{Y} \operatorname{Li}_{2}(-y)+\frac{1}{3} \tilde{Y}^{3}+\frac{1}{2} i \pi\left(Y^{2}+2 \pi^{2}\right)-\right. \\
& \left.-\frac{1}{2}\left(\tilde{Y}^{2}+\pi^{2}\right)(X-Y)-\frac{5}{8} \pi^{2} \tilde{Y}-\frac{\zeta_{3}}{6}\right]- \\
& -\frac{9-13 x}{48 y}\left(20 \tilde{Y}^{3}+17 \pi^{2} \tilde{Y}+20 \zeta_{3}\right)+ \\
& +\frac{1}{8}\left(11 \frac{y^{2}}{x^{2}}+\frac{4}{x}+26+\frac{88}{9} y\right)\left(\tilde{Y}^{2}+\pi^{2}\right)+\frac{\pi^{2}}{216}\left(396 \frac{x}{y}+63-217 y\right)- \\
& -\frac{1}{108}\left(297 \frac{x-y}{x}-56 y\right) \tilde{Y}-\frac{x}{18 y}+\frac{115}{72}-\frac{4849}{1296} y, \\
& C_{-+-+}^{[3]}=\frac{2 x^{2}-6 x+3}{y^{2}}\left[\operatorname{Li}_{4}\left(-\frac{x}{y}\right)-\mathrm{Li}_{4}(-x)-\mathrm{Li}_{4}(-y)+(X-Y) \mathrm{Li}_{3}(-x)-\right. \\
& -\frac{\pi^{2}}{6}\left(\operatorname{Li}_{2}(-x)-X^{2}-\frac{1}{2} Y^{2}\right)+\frac{1}{24} X^{4}-\frac{1}{6} X Y^{3}+ \\
& \left.+\frac{1}{24} Y^{4}-\zeta_{3}(X-Y)+\frac{7}{120} \pi^{4}\right]+ \\
& +\frac{1}{6}\left(2 \frac{y}{x}-3 \frac{9 x-13}{y}\right)\left[\operatorname{Li}_{3}(-x)+\operatorname{Li}_{3}(-y)-(X-Y) \operatorname{Li}_{2}(-x)\right]- \\
& -\frac{y}{18 x}\left[(2 X-Y)\left((X+Y)^{2}-6 Y^{2}\right)+6 i \pi\left((X-Y)^{2}+\pi^{2}\right)+\right. \\
& \left.+\frac{\pi^{2}}{4}(17 X+25 Y+2 i \pi)+5 \zeta_{3}\right]+ \\
& +\frac{9 x-13}{12 y}\left(X^{3}-3 X Y^{2}+2 \pi^{2}(X+Y)\right)+\frac{11}{9} \frac{y}{x}\left(\tilde{Y}^{2}+\pi^{2}\right)+ \\
& +\frac{1}{8}\left(11 y^{2}+4 x+26\right)\left((X-Y)^{2}+\pi^{2}\right)-\frac{\pi^{2}}{216}\left(217 \frac{y}{x}-63-\frac{396}{y}\right)+ \\
& +\frac{193}{108} \frac{y}{x} \tilde{Y}+\frac{1}{108}\left(297(1-y)+137 \frac{y}{x}\right)(X-Y)-\frac{1}{18 y}+ \\
& +\frac{115}{72}-\frac{4849}{1296} \frac{y}{x}
\end{aligned}
$$




$$
\begin{aligned}
& D_{-+-+}^{[1]}=-A_{-+-+}^{\mathrm{SYM},[1]}+A_{-+-+}^{[1]}+C_{-+-+}^{[1]}+E_{-+-+}^{[1]}+ \\
& +\frac{1+x^{2} y}{3 y^{2}}\left(\tilde{X}^{2}+\pi^{2}\right)(\tilde{X}-2 \tilde{Y})-\frac{y}{3 x}(4-y) \tilde{X} \tilde{Y}^{2}-\frac{2}{3} \frac{y^{2}}{x}\left(\tilde{Y}^{2}+\pi^{2}\right) \tilde{Y}+ \\
& +\frac{2}{3} \frac{y}{x}\left(\tilde{X}^{2}-\pi^{2}\right) \tilde{Y}-\frac{\pi^{2}}{3} y \tilde{X}-\frac{1}{9}\left(\frac{6}{y}-3 x+8 y\right) \tilde{X}^{2}+ \\
& +\frac{y^{2}}{9 x}\left(22 \tilde{Y}^{2}+\frac{5}{16} \pi^{2}\right)+\frac{1}{9}\left(\frac{12}{y}-6 x+16 y-11 \frac{y^{2}}{x}\right) \tilde{X} \tilde{Y}+ \\
& +\frac{1}{3}(\tilde{X}-2 \tilde{Y})-\frac{17}{54} \frac{y^{2}}{x}, \\
& D_{-+-+}^{[2]}=-A_{-+-+}^{\mathrm{SYM},[2]}+A_{-+-+}^{[2]}+C_{-+-+}^{[2]}+E_{-+-+}^{[2]}- \\
& -\frac{1}{3} \frac{1+x^{2} y}{y^{2}}\left(\tilde{X}^{2}+\pi^{2}\right)(2 \tilde{X}-\tilde{Y})-\frac{y^{2}}{3 x} \tilde{Y}^{2}(4 X-Y+3 i \pi)- \\
& -\frac{4}{3} \frac{y}{x} \tilde{X}^{2} \tilde{Y}-\frac{y}{3}\left(2 \tilde{X} \tilde{Y}^{2}-\pi^{2}(2 \tilde{X}-\tilde{Y})\right)+ \\
& +\frac{2}{9}\left(\frac{6}{y}-3 x+8 y\right) \tilde{X}^{2}-\frac{11}{9} \frac{y^{2}}{x} \tilde{Y}^{2}-\frac{1}{9}\left(\frac{6}{y}-3 x+8 y-22 \frac{y^{2}}{x}\right) \tilde{X} \tilde{Y}+ \\
& +\frac{5}{144} \pi^{2} y+\frac{1}{3}(\tilde{Y}-2 \tilde{X})-\frac{17}{54} y, \\
& D_{-+-+}^{[3]}=-A_{-+-+}^{\mathrm{SYM},[3]}+A_{-+-+}^{[3]}+C_{-+-+}^{[3]}+E_{-+-+}^{[3]}+ \\
& +\frac{1+x^{2} y}{3 y^{2}}\left(\tilde{X}^{2}+\pi^{2}\right)(\tilde{X}+\tilde{Y})+\frac{y^{2}}{3 x}\left(\left(\tilde{Y}^{2}+\pi^{2}\right) \tilde{Y}+\tilde{X} \tilde{Y}^{2}\right)+ \\
& +\frac{2}{3} \frac{y}{x}\left(\left(\tilde{X}^{2}+\frac{\pi^{2}}{2}\right) \tilde{Y}+\tilde{X} \tilde{Y}^{2}\right)-\frac{\pi^{2}}{3} y \tilde{X}- \\
& -\frac{1}{9}\left(\frac{6}{y}-3 x+8 y\right) \tilde{X}^{2}-\frac{11}{9} \frac{y^{2}}{x} \tilde{Y}^{2}+\frac{1}{9}\left(6 \frac{x}{y}+3+11 \frac{y}{x}\right) \tilde{X} \tilde{Y}+\frac{5}{144} \pi^{2} \frac{y}{x}+ \\
& +\frac{1}{3}(\tilde{X}+\tilde{Y})-\frac{17}{54} \frac{y}{x} \\
& E_{-+-+}^{[1]}=-\frac{1+x^{2}}{12 y^{2}}\left(\tilde{X}^{2}+\pi^{2}\right) \tilde{X}+\frac{1}{18}\left(\frac{x^{2}+5}{y}-x\right) \tilde{X}^{2}-\pi^{2} \frac{y^{2}}{108 x}-\frac{1}{6} \tilde{X} \\
& E_{-+-+}^{[2]}=-\frac{y}{9}\left(\tilde{Y}^{2}+\frac{\pi^{2}}{12}\right)-\frac{1-x}{72 y}, \\
& E_{-+-+}^{[3]}=-\frac{1}{9} \frac{y}{x}\left(\tilde{Y}^{2}+\frac{\pi^{2}}{12}\right)+\frac{1-x}{72 y}, \\
& F_{-+-+}^{[1]}=\frac{1}{18}\left[\frac{y^{2}}{x}\left(\tilde{X}^{2}+4 \pi^{2}\right)+\frac{y}{x}(2-y)\left(\tilde{Y}^{2}+\pi^{2}\right)+\right. \\
& \left.+\frac{y}{x}(2 x-y)\left((X-Y)^{2}+\pi^{2}\right)\right] \\
& F_{-+-+}^{[2]}=\frac{1}{18}\left[\frac{y}{x}(2-x)\left(\tilde{X}^{2}+\pi^{2}\right)+y\left(\tilde{Y}^{2}+4 \pi^{2}\right)+\right.
\end{aligned}
$$




$$
\begin{aligned}
& \left.+\frac{y}{x}(2 y-x)\left((X-Y)^{2}+\pi^{2}\right)\right] \\
& F_{-+-+}^{[3]}=\frac{1}{18}\left[-\frac{y}{x}(1-2 x)\left(\tilde{X}^{2}+\pi^{2}\right)-\frac{y}{x}(1-2 y)\left(\tilde{Y}^{2}+\pi^{2}\right)+\right. \\
& \left.+\frac{y}{x}\left((X-Y)^{2}+4 \pi^{2}\right)\right] \\
& H_{-+-+}^{[7]}=-\frac{1+x^{2}}{y^{2}}\left[6 \operatorname{Li}_{4}(-x)-4 \tilde{X}\left(\operatorname{Li}_{3}(-x)+\operatorname{Li}_{3}(-y)-\zeta_{3}\right)-2 \tilde{Y}\left(\operatorname{Li}_{3}(-x)-\zeta_{3}\right)+\right. \\
& +\left(\tilde{X}^{2}-2 \tilde{X} \tilde{Y}+\pi^{2}\right) \operatorname{Li}_{2}(-x)+\frac{1}{3} X^{3} \tilde{Y}-2 \tilde{X} X Y^{2}- \\
& \left.-\frac{\pi^{2}}{12}\left(9 X^{2}-20 X Y-2 i \pi(X+2 Y)\right)-\frac{2}{5} \pi^{4}\right]+ \\
& +2\left(\frac{14}{y^{2}}+\frac{16}{y}+5\right)\left[\operatorname{Li}_{4}\left(-\frac{x}{y}\right)+\operatorname{Li}_{4}(-x)-\operatorname{Li}_{4}(-y)-\tilde{Y}\left(\operatorname{Li}_{3}(-x)-\zeta_{3}\right)+\right. \\
& +\frac{\pi^{2}}{6} \operatorname{Li}_{2}(-x)-\frac{1}{6} X Y^{3}+\frac{1}{24}\left(Y^{2}+2 \pi^{2}\right) Y^{2}- \\
& \left.-\frac{7}{360} \pi^{4}\right]- \\
& -\frac{1}{3 y^{2}}\left(14-x^{2}(1-2 x)\right)\left(\operatorname{Li}_{3}(-x)-\zeta_{3}-\tilde{X} \operatorname{Li}_{2}(-x)+\frac{1}{2} i \pi\left(\tilde{X}^{2}+\pi^{2}\right)\right)- \\
& -\frac{2}{3}\left(30 \frac{x}{y}-x+8\right)\left(\operatorname{Li}_{3}(-y)-\zeta_{3}-\tilde{Y} \operatorname{Li}_{2}(-y)-\frac{1}{2} X\left(\tilde{Y}^{2}+\pi^{2}\right)\right)+ \\
& +\frac{1+x^{2} y}{18 y^{2}}\left(4 \tilde{X}^{3}-7 \pi^{2} \tilde{X}+15\left(\tilde{X}^{2}+\pi^{2}\right) \tilde{Y}\right)+ \\
& +\frac{y^{2}}{36 x}\left(8 \tilde{Y}^{2}-12 \tilde{X} \tilde{Y}+24 \tilde{X}^{2}+29 \pi^{2}\right) \tilde{Y}- \\
& -\frac{1}{12}(23-10 x)\left(\tilde{X}^{2} Y+i \pi\left(X^{2}+\pi^{2}\right)\right)+ \\
& +\frac{\pi^{2}}{36}\left(50 \frac{x^{2}}{y}-45 x+117\right) \tilde{X}+\frac{\pi^{2}}{36}\left(\frac{96}{y}+27 x-7 y\right) \tilde{Y}+2 \frac{1-x}{y} \zeta_{3}+ \\
& +\frac{1}{36}\left(\frac{63}{y^{2}}+\frac{156}{y}+96-44 y+9 y^{2}\right)\left(\tilde{X}^{2}+\pi^{2}\right)+ \\
& +\frac{1}{36}\left(\frac{36}{x^{2}}+\frac{64}{x}+173-26 x+9 x^{2}\right)\left(\tilde{Y}^{2}+\pi^{2}\right)- \\
& -\frac{1}{18}\left(\frac{36}{y}-\frac{22}{x}+92+9 y^{2}\right) \tilde{X} \tilde{Y}-\frac{\pi^{2}}{36}(2-3 x)\left(\frac{26}{y}-\frac{22}{x}-5+3 y\right)- \\
& -\frac{1}{54}\left(45 \frac{x}{y}+9 x+92 y\right) \tilde{X}-\frac{1}{54}\left(92 \frac{y^{2}}{x}-9 x+153\right) \tilde{Y}, \\
& H_{-+-+}^{[8]}=\frac{4}{3}\left(\frac{x}{y^{2}}-2\right)\left(\operatorname{Li}_{3}(-x)-\zeta_{3}-\tilde{X} \operatorname{Li}_{2}(-x)+\frac{1}{2} i \pi\left(\tilde{X}^{2}+\pi^{2}\right)\right)+
\end{aligned}
$$




$$
\begin{aligned}
& +\frac{2}{9} \frac{1+x^{2} y}{y^{2}}\left(\tilde{X}^{3}+3\left(\tilde{X}^{2}+\pi^{2}\right) \tilde{Y}\right)-(1-y) \frac{y}{3 x}\left(\tilde{X}^{2}-2 \pi^{2}\right) \tilde{Y}+ \\
& +(5-2 y) \frac{y}{3 x} \tilde{X} \tilde{Y}^{2}+\frac{11}{36} \frac{y^{2}}{x}\left(4 \tilde{Y}^{2}+7 \pi^{2}\right) \tilde{Y}+\frac{29}{36} y \pi^{2} \tilde{X}- \\
& -\frac{1}{9}\left(\frac{9}{y^{2}}+\frac{15}{y}-(3-y)(7 x-2 y)\right)\left(\tilde{X}^{2}+\pi^{2}\right)+ \\
& +y^{2}\left(\frac{y^{2}}{x^{2}}-\frac{53}{9 x}\right)\left(\tilde{Y}^{2}+\pi^{2}\right)-\frac{y}{9}\left(\frac{11}{x}+3(y-5 x)\right)\left(\tilde{X} \tilde{Y}+4 \pi^{2}\right)+ \\
& +\frac{\pi^{2}}{3}\left(\frac{2}{y}+x^{2}-10 y(x-y)\right)-\frac{2}{27}\left(9 \frac{x}{y}+23 y\right) \tilde{X}-\frac{46}{27} \frac{y^{2}}{x} \tilde{Y}+\frac{1}{2}, \\
& H_{-+-+}^{[9]}=-\frac{1+x^{2}}{y^{2}}\left[6 \operatorname{Li}_{4}(-x)-4 \tilde{X}\left(\operatorname{Li}_{3}(-x)+\operatorname{Li}_{3}(-y)-\zeta_{3}\right)-2 \tilde{Y}\left(\operatorname{Li}_{3}(-x)-\zeta_{3}\right)+\right. \\
& +\left(\tilde{X}^{2}-2 \tilde{X} \tilde{Y}+\pi^{2}\right) \operatorname{Li}_{2}(-x)+\frac{1}{3} X^{3} \tilde{Y}-2 \tilde{X} X Y^{2}- \\
& \left.-\frac{\pi^{2}}{12}\left(9 X^{2}-20 X Y-2 i \pi(X+2 Y)\right)-\frac{2}{5} \pi^{4}\right]+ \\
& +2\left(14 \frac{x^{2}}{y^{2}}+16 \frac{x}{y}+5\right)\left[\operatorname{Li}_{4}\left(-\frac{x}{y}\right)+\operatorname{Li}_{4}(-x)-\operatorname{Li}_{4}(-y)-\tilde{Y} \operatorname{Li}_{3}(-x)+\right. \\
& +\tilde{Y} \zeta_{3}+\frac{\pi^{2}}{6} \operatorname{Li}_{2}(-x)-\frac{1}{6} X Y^{3}+ \\
& \left.+\frac{1}{24}\left(Y^{2}+2 \pi^{2}\right) Y^{2}-\frac{7}{360} \pi^{4}\right]- \\
& -8 \frac{1-3 x}{y^{2}}\left[\operatorname{Li}_{4}(-x)-\frac{1}{2} \tilde{X}\left(\operatorname{Li}_{3}(-x)-\zeta_{3}\right)+\frac{\pi^{2}}{6} \operatorname{Li}_{2}(-x)-\frac{1}{48} X^{4}-\right. \\
& \left.-\frac{\pi^{2}}{12} X^{2}-\frac{7}{180} \pi^{4}\right]- \\
& -\frac{1}{3}\left(\frac{11}{y^{2}}-\frac{30}{y}-2+\frac{4}{x}\right)\left[\operatorname{Li}_{3}(-x)-\zeta_{3}-\tilde{X}\left(\operatorname{Li}_{2}(-x)-\frac{\pi^{2}}{6}\right)+\right. \\
& \left.+\frac{1}{2} i \pi\left(\tilde{X}^{2}+\pi^{2}\right)\right]- \\
& -\frac{2}{3}\left(30 \frac{x}{y}-\frac{y}{x}+21\right)\left[\operatorname{Li}_{3}(-y)+\zeta_{3}-\tilde{Y}\left(\operatorname{Li}_{2}(-y)+\frac{\pi^{2}}{24}\right)-\right. \\
& \left.-\frac{1}{2} X\left(\tilde{Y}^{2}+\pi^{2}\right)\right]+ \\
& +\frac{1}{36}\left(\frac{30}{y^{2}}-\frac{14}{y}-44 x+39\right)\left(\tilde{X}^{2}+\pi^{2}\right) \tilde{X}- \\
& -\frac{1}{12}\left(10 \frac{x}{y^{2}}-13 x+3 y\right)\left(\tilde{X}^{2}+\pi^{2}\right) \tilde{Y}+
\end{aligned}
$$




$$
\begin{aligned}
& +\frac{y^{2}}{9 x}\left(2 \tilde{Y}^{3}-6 \tilde{X}^{2} \tilde{Y}+3 \tilde{X} \tilde{Y}^{2}-\pi^{2} \tilde{X}+8 \pi^{2} \tilde{Y}\right)+ \\
& +\frac{\pi^{2}}{36}\left(\frac{108}{y}+19 x+49\right) \tilde{X}+\frac{\pi^{2}}{12} \frac{x}{y}(22-17 y) \tilde{Y}-2 \frac{1-x}{y} \zeta_{3}+ \\
& +\frac{1}{36}\left(\frac{63}{y^{2}}-\frac{6}{y}+3 x^{2}-33 x y-251 y\right)\left(\tilde{X}^{2}+\pi^{2}\right)+ \\
& +\frac{1}{36}\left(\frac{9}{x^{2}}-\frac{26}{x}+173-36 x y+28 x\right)\left(\tilde{Y}^{2}+\pi^{2}\right)- \\
& -\frac{1}{9}\left(\frac{18}{y}+\frac{22}{x}-73 x-18 x y-142 y\right) \tilde{X} \tilde{Y}+ \\
& +\frac{\pi^{2}}{36}\left(\frac{106}{y}+\frac{44}{x}+11 x^{2}+47 x y+193 y\right)-\frac{1}{54}\left(45 \frac{x}{y}-144+92 y\right) \tilde{X}- \\
& -\frac{1}{54}\left(92 \frac{y^{2}}{x}-\frac{9}{x}+153\right) \tilde{Y} \\
& I_{-+-+}^{[7]}=\frac{1}{18}\left[3 \frac{x^{2}+1}{y^{2}}\left(\tilde{X}^{2}+\pi^{2}\right) \tilde{Y}-\left(\frac{1}{x}+2 x-6 \frac{x}{y}\right)\left(\tilde{X}^{2}+\pi^{2}\right)+\right. \\
& +\left(-\frac{6}{y}+2 x+\frac{1}{x}\right)\left(\tilde{Y}^{2}+\pi^{2}\right)+\left(\frac{1}{x}+2 \frac{1-2 x}{y}\right)\left((X-Y)^{2}+\pi^{2}\right)+ \\
& \left.+\left(\frac{y}{x}-3-6 \frac{x}{y}\right) \pi^{2}+6 \tilde{Y}\right] \\
& I_{-+-+}^{[8]}=\frac{1}{9}\left(y(X-Y)(\tilde{X}+4 \tilde{Y})+\frac{y}{x}(\tilde{X}-4 \tilde{Y}) \tilde{Y}\right), \\
& I_{-+-+}^{[9]}=\frac{1}{18}\left[-3 \frac{x^{2}+1}{y^{2}}(X-Y)\left(\tilde{X}^{2}+\pi^{2}\right)+2\left(2 \frac{y^{2}}{x}+3 y\right)\left(\tilde{X}^{2}+\pi^{2}\right)+\right. \\
& +2 \frac{y^{2}}{x}\left(\tilde{Y}^{2}+\pi^{2}\right)+2\left(2 \frac{y}{x}-3 x+\frac{6}{y}\right)(X-Y) \tilde{X}+ \\
& \left.+6 \pi^{2} \frac{y}{x}-6(X-Y)\right] \text {. }
\end{aligned}
$$

\section{B. Finite remainder functions for pure $N=1$ super-Yang-Mills theory}

In this appendix, we present the independent $N=1$ supersymmetric finite remainder functions appearing in eqs. (6.11) and (6.12). For --++ , these functions are

$$
\begin{aligned}
A_{--++}^{\mathrm{SYM},[1]}=3 y[ & \operatorname{Li}_{4}(-x)+\operatorname{Li}_{4}(-y)-\operatorname{Li}_{4}\left(-\frac{x}{y}\right)-\tilde{X}\left(\operatorname{Li}_{3}(-y)+\operatorname{Li}_{3}(-x)\right)+ \\
& +\frac{\pi^{2}}{6} \operatorname{Li}_{2}(-x)-\frac{1}{6} X^{3} Y+\frac{1}{24}\left(\tilde{X}^{4}-\tilde{Y}^{4}\right)-\frac{1}{12} \tilde{X} \tilde{Y}^{2}(3 \tilde{X}-2 \tilde{Y})- \\
& \left.-\frac{\pi^{2}}{12}\left((X+Y)^{2}+2 i \pi Y\right)-\frac{\pi^{4}}{180}\right]-
\end{aligned}
$$




$$
\begin{aligned}
& -\frac{1}{2 x}\left(\frac{1}{4} \tilde{X}^{4}+\frac{\pi^{2}}{3} \tilde{X}^{2}-\zeta_{3} \tilde{X}+\frac{\pi^{4}}{40}\right)+ \\
& +\frac{3}{2} \frac{1-2 x}{x}\left[\operatorname{Li}_{3}(-x)-\zeta_{3}-\tilde{X}\left(\operatorname{Li}_{2}(-x)+\frac{2}{3} \pi^{2}\right)+\frac{1}{6} \tilde{X}^{3}-\right. \\
& \left.-\frac{1}{2}\left(\tilde{X}^{2}+\pi^{2}\right) \tilde{Y}+i \frac{\pi}{2}\left(X^{2}+2 \pi^{2}\right)\right]+ \\
& +\frac{1}{16 x}\left(4 \tilde{X}^{3}+9 \pi^{2} \tilde{X}-52 \zeta_{3}\right)+\frac{3}{2 y}\left(\tilde{X}^{2}+\pi^{2}\right)- \\
& -\frac{\pi^{2}}{16 x}(13-8 x)-\frac{85}{18 x} \tilde{X}+\frac{143}{12 x}, \\
& A_{--++}^{\mathrm{SYM},[3]}=-\frac{x}{8 y}\left((X-Y)^{2}+\pi^{2}\right)^{2}-\frac{\pi^{2}}{12}\left(\frac{1}{y}-3 x\right)\left((X-Y)^{2}+\pi^{2}\right)+ \\
& +\frac{\zeta_{3}}{2} \frac{\tilde{X}}{x y}-\frac{7}{240} \frac{\pi^{4}}{y}-\frac{1}{8 y}(1-x)(y-3 x)\left(\tilde{X}^{2}-3 \tilde{X} \tilde{Y}+\frac{\pi^{2}}{3}\right) \tilde{X}- \\
& -\frac{3}{2 x y}\left[\operatorname{Li}_{3}(-x)-X\left(\operatorname{Li}_{2}(-x)-\frac{\pi^{2}}{6}\right)-\frac{1}{3} X^{3}-\frac{1}{2} X^{2} Y-\right. \\
& \left.-i \frac{\pi}{2}\left(X^{2}+X Y+Y^{2}\right)+\frac{5}{8} \pi^{2} X+i \frac{\pi^{3}}{24}+\frac{13}{12} \zeta_{3}\right]- \\
& -\frac{9}{4} \tilde{X}^{2} \tilde{Y}-\frac{\pi^{2}}{12}(7-4 x) \tilde{X}+\frac{9}{4 y}\left(\tilde{X}^{2}+\pi^{2}\right)+\frac{3}{4}\left((X-Y)^{2}+\pi^{2}\right)+ \\
& +\frac{\pi^{2}}{2}\left(\frac{23}{16 x y}-1\right)-\frac{3}{2 x y}\left(\frac{58}{27}-y\right) \tilde{X}-\frac{143}{12 y}+\{t \leftrightarrow u\}, \\
& B_{--++}^{\mathrm{SYM},[1]}=2 \frac{x(1-x)}{y}\left[\operatorname{Li}_{4}\left(-\frac{x}{y}\right)-4 \operatorname{Li}_{4}(-x)+\operatorname{Li}_{4}(-y)+3 \tilde{X} \operatorname{Li}_{3}(-x)-\right. \\
& -\frac{1}{2}\left(\tilde{X}^{2}+2(X-Y) \tilde{Y}+2 \pi^{2}\right) \operatorname{Li}_{2}(-x)-\frac{1}{48} \tilde{X}^{4}+\frac{1}{6} X^{3} Y+ \\
& +\frac{1}{24} Y^{4}-\frac{1}{8} X^{2} Y^{2}+\frac{1}{12}(X-i \pi) Y^{3}+i \frac{\pi}{4} X Y(3 X-2 Y)- \\
& \left.-\frac{\pi^{2}}{24}\left(9 X^{2}+18 X Y-7 Y^{2}+20 i \pi X\right)+\frac{83}{360} \pi^{4}\right]+ \\
& +4 \frac{1+x^{2}}{x}\left[\operatorname{Li}_{4}\left(-\frac{x}{y}\right)+2 \operatorname{Li}_{4}(-x)-2 \operatorname{Li}_{4}(-y)+\frac{3}{2} \tilde{X} \operatorname{Li}_{3}(-y)-\right. \\
& -\frac{3}{2} \tilde{Y} \operatorname{Li}_{3}(-x)-\frac{1}{2}\left((X-Y)^{2}-2 \tilde{X} \tilde{Y}-\pi^{2}\right) \operatorname{Li}_{2}(-x)- \\
& -\frac{3}{8} X^{3} Y+\frac{17}{16} X^{2} Y^{2}-\frac{5}{24} X Y^{3}-\frac{3}{2} \zeta_{3}(X-Y)-\frac{29}{720} \pi^{4}- \\
& -i \frac{\pi}{24}\left(X^{3}+18 X^{2} Y-42 X Y^{2}+Y^{3}\right)+ \\
& \left.+\frac{\pi^{2}}{48}\left(27 X^{2}-36 X Y+11 Y^{2}+2 i \pi(11 X-Y)\right)\right]-
\end{aligned}
$$




$$
\begin{aligned}
& -6 x\left[2 \operatorname{Li}_{4}\left(-\frac{x}{y}\right)+3 \mathrm{Li}_{4}(-x)+2 \operatorname{Li}_{4}(-y)-\tilde{Y}\left(3 \operatorname{Li}_{3}(-x)+2 \mathrm{Li}_{3}(-y)\right)-\right. \\
& -\frac{1}{4}\left((X-Y)^{2}-2 \tilde{X} \tilde{Y}+(X-Y)(\tilde{X}+\tilde{Y})+\frac{2}{3} \pi^{2}\right) \operatorname{Li}_{2}(-x)- \\
& -\frac{13}{36} X^{3} Y+\frac{5}{12} X^{2} Y^{2}-\frac{11}{18} X Y^{3}+\frac{5}{36} Y^{4}- \\
& -i \frac{\pi}{36}\left(X^{3}+27 X^{2} Y-10 Y^{3}\right)+\frac{\pi^{2}}{12}\left(5 X(X-Y)+2 Y^{2}\right)+ \\
& \left.+i \frac{\pi^{3}}{36}(13 X+11 Y)-\zeta_{3}(X-Y)-\frac{59}{360} \pi^{4}\right]-\frac{1}{24}(3 x-2 y) \tilde{X}^{4}- \\
& -2\left[4 \operatorname{Li}_{4}\left(-\frac{x}{y}\right)+5 \operatorname{Li}_{4}(-x)-5 \operatorname{Li}_{4}(-y)-6 \tilde{Y} \operatorname{Li}_{3}(-x)-\right. \\
& -\frac{1}{2}\left((X-Y)^{2}-2 \tilde{X} \tilde{Y}-2 \pi^{2}\right) \operatorname{Li}_{2}(-x)+X^{2} Y^{2}-\frac{5}{6} X^{3} Y- \\
& -\frac{7}{6} X Y^{3}+\frac{5}{12} Y^{4}-i \frac{\pi}{6}\left(3 X^{3}+9 X^{2} Y-6 X Y^{2}-4 Y^{3}\right)+ \\
& \left.+\frac{\pi^{2}}{24}\left(27 X^{2}+18 X Y-11 Y^{2}+4 i \pi(4 X-3 Y)\right)-\frac{11}{180} \pi^{4}\right]- \\
& -3\left(\frac{2 x^{2}}{y}-x-\frac{2}{x}\right)\left(\operatorname{Li}_{3}(-x)-\zeta_{3}-\tilde{X} \operatorname{Li}_{2}(-x)+i \frac{\pi}{2} \tilde{X}^{2}+i \frac{\pi^{3}}{2}\right)- \\
& -3\left(\frac{4}{y}+2 y-\frac{y^{2}}{x}\right)\left(\operatorname{Li}_{3}(-y)-\zeta_{3}-\tilde{Y} \operatorname{Li}_{2}(-y)+i \frac{\pi}{2} \tilde{Y}^{2}+i \frac{\pi^{3}}{2}\right)+ \\
& +\frac{1}{4}\left(3 x \frac{1-x}{y}-y\right) \tilde{X}^{3}+\frac{3}{4}\left(5(1-y) \frac{y}{x}-3 x\right) \tilde{X} \tilde{Y}^{2}-3 x \frac{1-x}{y} \tilde{X}^{2} \tilde{Y}- \\
& -\frac{1}{2}\left(3 y \frac{1-y}{x}-x\right) \tilde{Y}^{3}+\frac{\pi^{2}}{4}\left(\frac{4 x^{2}}{y}+2 x-37\right) \tilde{X}+6 \zeta_{3}\left(\frac{y^{2}}{x}-2 \frac{x^{2}}{y}\right)- \\
& -\frac{\pi^{2}}{2}\left(\frac{y^{2}}{x}+2 y-19\right) \tilde{Y}+\frac{3}{4}\left(7 \frac{x^{2}}{y}+x-3\right) \tilde{X}^{2}+\frac{3}{2}\left(4 \frac{y}{x}-3 x\right) \tilde{Y}^{2}+ \\
& +\frac{3}{4}(y-2 x)\left(\frac{3}{x y}-4\right) \tilde{X} \tilde{Y}-\frac{3}{2} \pi^{2}(y-2 x)\left(\frac{1}{x y}-1\right) \text {. }
\end{aligned}
$$

The independent $N=1$ supersymmetric remainder functions for -+-+ are,

$$
\begin{aligned}
A_{-+-+}^{\mathrm{SYM},[1]}= & -\frac{1+x^{2}}{8 x}\left(\tilde{X}^{2}+\pi^{2}\right)^{2}+\frac{\pi^{2}}{12}\left(\frac{y^{2}}{x}-3\right)\left(\tilde{X}^{2}+\pi^{2}\right)+\frac{y^{2}}{2 x}\left(\zeta_{3} \tilde{X}+\frac{7}{120} \pi^{4}\right)+ \\
+ & \frac{3}{2} \frac{y^{2}}{x}\left[\operatorname{Li}_{3}(-x)-\tilde{X} \operatorname{Li}_{2}(-x)+\frac{1}{3} \tilde{X}^{3}-\frac{1}{2}\left(\tilde{X}^{2}+\pi^{2}\right) Y+\frac{17}{24} \pi^{2} \tilde{X}-\right. \\
& \left.\quad-\frac{19}{6} \zeta_{3}\right]+\frac{1}{8 y}(x-y)(1-3 x)\left(\tilde{X}^{2}+\pi^{2}\right) \tilde{X}+\frac{\pi^{2}}{2} y \tilde{X}- \\
& -\frac{3}{4}(2 x-y)\left(\tilde{X}^{2}+\pi^{2}\right)+\frac{\pi^{2}}{16}\left(13 \frac{y}{x}-7 y+16 x\right)+
\end{aligned}
$$




$$
\begin{aligned}
& +\frac{3}{2} \frac{y}{x}\left(1-\frac{58}{27} y\right) \tilde{X}+\frac{143}{12} \frac{y^{2}}{x} \\
& A_{-+-+}^{\mathrm{SYM},[2]}=3 \frac{x}{y}\left[\operatorname{Li}_{4}\left(-\frac{x}{y}\right)+\mathrm{Li}_{4}(-x)-\mathrm{Li}_{4}(-y)-\tilde{Y}\left(\mathrm{Li}_{3}(-x)-\zeta_{3}\right)+\right. \\
& \left.+\frac{\pi^{2}}{6} \operatorname{Li}_{2}(-x)+\frac{1}{24}\left(Y^{2}-4 X Y+2 \pi^{2}\right) Y^{2}-\frac{7}{360} \pi^{4}\right]- \\
& -\frac{y}{8}\left(\tilde{Y}^{4}+\frac{4}{3} \pi^{2} \tilde{Y}^{2}-4 \zeta_{3} \tilde{Y}+\frac{\pi^{4}}{10}\right)+\frac{3}{4}(2-3 x) \frac{y}{x}\left(\tilde{Y}^{2}+\pi^{2}\right)- \\
& -\frac{3}{2}(2-y)\left[\operatorname{Li}_{3}(-y)-\tilde{Y}\left(\operatorname{Li}_{2}(-y)-\frac{\pi^{2}}{6}\right)-\frac{1}{2} X\left(\tilde{Y}^{2}+\pi^{2}\right)-\zeta_{3}\right]- \\
& -\frac{\pi^{2}}{16}(8 x-15 y)+\frac{y}{16}\left(20 \tilde{Y}^{3}+17 \pi^{2} \tilde{Y}-52 \zeta_{3}\right)-\frac{29}{9} y \tilde{Y}+\frac{143}{12} y, \\
& A_{-+-+}^{\mathrm{SYM},[3]}=\frac{3}{y}\left[\operatorname{Li}_{4}\left(-\frac{x}{y}\right)-\mathrm{Li}_{4}(-x)-\mathrm{Li}_{4}(-y)+(X-Y)\left(\operatorname{Li}_{3}(-x)-\zeta_{3}\right)-\right. \\
& \left.-\frac{\pi^{2}}{6}\left(\operatorname{Li}_{2}(-x)-X^{2}-\frac{1}{2} Y^{2}\right)+\frac{1}{6} X^{3} Y-\frac{1}{4} X^{2} Y^{2}+\frac{7}{120} \pi^{4}\right]- \\
& -\frac{y}{2 x}\left(\frac{\pi^{2}}{3}(X-Y)^{2}+\frac{\pi^{4}}{40}-\zeta_{3}(\tilde{X}+\tilde{Y})\right)-\frac{1}{8} \frac{1-x y}{x y}(X-Y)^{4}- \\
& -\frac{3}{2}\left(\frac{y}{x}-2\right)\left[\operatorname{Li}_{3}(-x)+\operatorname{Li}_{3}(-y)-(X-Y) \operatorname{Li}_{2}(-x)-\frac{1}{6} X^{3}+\right. \\
& \left.+\frac{1}{2} X Y^{2}-\frac{\pi^{2}}{3}(X+Y)\right]+ \\
& +\frac{y}{16 x}\left(4(\tilde{X}+5 \tilde{Y})(X-Y)^{2}+\pi^{2}(9 \tilde{X}+17 \tilde{Y})-52 \zeta_{3}\right)- \\
& -\frac{9}{4} \frac{y}{x}\left(\tilde{Y}^{2}+\pi^{2}\right)+\frac{3}{2} y\left((X-Y)^{2}+\pi^{2}\right)-\frac{\pi^{2}}{16 x}(8-15 y)- \\
& -\frac{y}{2 x}\left(\frac{85}{9} \tilde{X}+\frac{58}{9} \tilde{Y}\right)+\frac{143}{12} \frac{y}{x} \\
& B_{-+-+}^{\mathrm{SYM},[1]}=4\left(\frac{y^{2}}{x}-3\right)\left[\operatorname{Li}_{4}\left(-\frac{x}{y}\right)+2 \operatorname{Li}_{4}(-x)-2 \operatorname{Li}_{4}(-y)-\frac{3}{2} \tilde{Y}\left(\operatorname{Li}_{3}(-x)-\zeta_{3}\right)+\right. \\
& +\frac{3}{2} \tilde{X}\left(\operatorname{Li}_{3}(-y)-\zeta_{3}\right)-\frac{1}{2}\left((X-Y)^{2}-\pi^{2}-2 \tilde{X} \tilde{Y}\right) \operatorname{Li}_{2}(-x)- \\
& -\frac{3}{8} X^{3} Y+X^{2} Y^{2}-i \frac{\pi}{24} X^{3}+i \pi X Y^{2}+ \\
& \left.+\frac{\pi^{2}}{24}\left(X^{2}-20 X Y+5 Y^{2}-2 i \pi(X+2 Y)\right)-\frac{\pi^{4}}{240}\right]+ \\
& +12\left(\frac{x}{y}+2(1-x)\right)\left[\operatorname{Li}_{4}(-x)-\frac{1}{2} \tilde{X}\left(\operatorname{Li}_{3}(-x)-\zeta_{3}\right)+\right.
\end{aligned}
$$




$$
\begin{aligned}
& \left.+\frac{\pi^{2}}{6}\left(\operatorname{Li}_{2}(-x)-\frac{1}{2} X^{2}\right)-\frac{1}{48} X^{4}-\frac{7}{180} \pi^{4}\right]- \\
& -6\left[3 \operatorname{Li}_{4}\left(-\frac{x}{y}\right)+3 \operatorname{Li}_{4}(-x)-\mathrm{Li}_{4}(-y)+\tilde{X} \operatorname{Li}_{3}(-y)+\right. \\
& +\left((X-Y)^{2}-\tilde{X}^{2}+\frac{\pi^{2}}{6}\right) \operatorname{Li}_{2}(-x)+ \\
& \left.+\frac{5}{24}\left(2 X^{3}-4 X Y^{2}+Y^{3}\right) Y-\frac{1}{6} X^{4}-\frac{1}{4} i \pi X^{3}+3 \zeta_{3} \tilde{X}\right]- \\
& -4 y\left[\operatorname{Li}_{4}\left(-\frac{x}{y}\right)+2 \operatorname{Li}_{4}(-x)+\operatorname{Li}_{4}(-y)+\frac{1}{24}\left(4 X^{3}-8 X Y^{2}+Y^{3}\right) Y-\right. \\
& -\frac{7}{48} X^{4}+\frac{1}{2}\left((X-Y)^{2}-2 \tilde{X}^{2}+\tilde{Y}^{2}\right) \operatorname{Li}_{2}(-x)- \\
& -i \frac{\pi}{12}(X-Y)\left(X^{2}+10 X Y-2 Y^{2}\right)+3 \zeta_{3} \tilde{X}+ \\
& \left.+\frac{\pi^{2}}{24}\left(7 X(X-2 Y)+8 Y^{2}+2 i \pi(4 X+3 Y)\right)-\frac{23}{240} \pi^{4}\right]- \\
& -(1-x) \frac{y}{12 x}\left[(3 X-10 Y) X Y^{2}-2 i \pi\left(18(X-Y) X+Y^{2}\right) Y+\right. \\
& \left.+\pi^{2}\left(25 X^{2}+4 X Y+Y^{2}+2 i \pi(13 X+3 Y)\right)-\frac{26}{15} \pi^{4}\right]+ \\
& +3 \frac{1+y^{2}}{x}\left[\operatorname{Li}_{3}(-x)-\tilde{X} \operatorname{Li}_{2}(-x)-\frac{3}{4} \tilde{X}^{2}(2 Y+i \pi)+\right. \\
& \left.+\frac{1}{12} X^{3}+\frac{\pi^{2}}{12}(2 X-10 Y-3 i \pi)-5 \zeta_{3}\right]- \\
& -3(1-x) \frac{y}{x}\left[\operatorname{Li}_{3}(-y)-\tilde{Y} \operatorname{Li}_{2}(-y)-\frac{1}{8} \tilde{X}^{3}+\frac{\pi^{2}}{8} \tilde{X}+\frac{5}{4} \tilde{X}^{2} \tilde{Y}-\right. \\
& \left.-\frac{1}{2}(2 X+i \pi) \tilde{Y}^{2}+\frac{5}{12} \pi^{2} \tilde{Y}+i \frac{\pi^{3}}{2}+2 \zeta_{3}\right]- \\
& -\frac{y^{2}}{8 x}\left[\tilde{X}^{3}-42 \tilde{X}^{2} \tilde{Y}+36 \tilde{X} \tilde{Y}^{2}-24 \tilde{Y}^{3}-\pi^{2}(\tilde{X}+26 \tilde{Y})-168 \zeta_{3}\right]- \\
& -\frac{x^{2}}{y}\left(\tilde{X}^{2}+\pi^{2}\right) \tilde{X}+\frac{3}{8}\left(3 \frac{y^{2}}{x}-4\right)\left(\tilde{X}^{2}+\pi^{2}\right)+\frac{3}{8} \frac{y}{x}(2-3 y)\left(\tilde{Y}^{2}+\pi^{2}\right)+ \\
& +\frac{3}{8} \frac{y}{x}(2 x-3 y)\left((X-Y)^{2}+\pi^{2}\right)+\frac{27 \pi^{2}}{8} \frac{y^{2}}{x}, \\
& B_{-+-+}^{\mathrm{SYM},[2]}=-\frac{6}{y}\left[3 \operatorname{Li}_{4}\left(-\frac{x}{y}\right)-\mathrm{Li}_{4}(-x)-3 \operatorname{Li}_{4}(-y)+\frac{1}{12} X^{4}-\frac{1}{2} X Y^{3}+\right.
\end{aligned}
$$




$$
\begin{aligned}
& +\frac{1}{8} Y^{4}+(2 \tilde{X}-3 \tilde{Y}) \operatorname{Li}_{3}(-x)-\frac{\pi^{2}}{6}\left(\operatorname{Li}_{2}(-x)-2 X^{2}-\frac{3}{2} Y^{2}\right)- \\
& \left.-\zeta_{3}(2 \tilde{X}-3 \tilde{Y})+\frac{7}{72} \pi^{4}\right]+ \\
& +4 \frac{y^{2}}{x}\left[\operatorname{Li}_{4}\left(-\frac{x}{y}\right)-\mathrm{Li}_{4}(-x)+4 \operatorname{Li}_{4}(-y)-3 \tilde{Y} \operatorname{Li}_{3}(-y)+\frac{1}{16} Y^{4}+\right. \\
& +\frac{1}{2}\left(2 \tilde{X}(X-Y)-\tilde{Y}^{2}-2 \pi^{2}\right) \operatorname{Li}_{2}(-x)+\frac{3}{4} X^{3} Y+ \\
& +i \frac{\pi}{12}\left(X^{3}+18 X^{2} Y-33 X Y^{2}+Y^{3}\right)-\frac{5}{8} X^{2} Y^{2}-\frac{5}{6} X Y^{3}- \\
& \left.-\frac{\pi^{2}}{24}\left(9 X^{2}-6 X Y-10 Y^{2}+i \pi(4 X-26 Y)\right)-\frac{77}{720} \pi^{4}\right]+ \\
& +6\left[\operatorname{Li}_{4}\left(-\frac{x}{y}\right)+2 \mathrm{Li}_{4}(-x)-\mathrm{Li}_{4}(-y)+2 \tilde{X} \operatorname{Li}_{3}(-y)-\right. \\
& -\frac{1}{2}\left(X^{2}-2 \tilde{X} Y+\frac{\pi^{2}}{3}\right) \operatorname{Li}_{2}(-x)-\frac{1}{12} X^{4}-\frac{1}{6} X^{3} Y+ \\
& +X^{2} Y^{2}-\frac{1}{6} X Y^{3}+\frac{1}{24} Y^{4}-i \frac{\pi}{6}\left(X^{2}-6 Y^{2}\right) X+ \\
& \left.+\frac{\pi^{2}}{24}\left(X^{2}-20 X Y+2 Y^{2}-2 i \pi(X+2 Y)\right)+\frac{\pi^{4}}{40}\right]- \\
& -4 y\left[\operatorname{Li}_{4}\left(-\frac{x}{y}\right)+2 \operatorname{Li}_{4}(-x)-2 \operatorname{Li}_{4}(-y)-\frac{3}{2} \tilde{Y}\left(\operatorname{Li}_{3}(-x)-\zeta_{3}\right)+\right. \\
& +\frac{3}{2} \tilde{X}\left(\operatorname{Li}_{3}(-y)-\zeta_{3}\right)+\frac{1}{24} X^{4}-\frac{11}{24} X^{3} Y+\frac{19}{16} X^{2} Y^{2}- \\
& -\frac{1}{2}\left((X-Y)^{2}-\pi^{2}-2 \tilde{X} \tilde{Y}\right) \operatorname{Li}_{2}(-x)- \\
& -\frac{7}{24} X Y^{3}+\frac{1}{24} Y^{4}+i \frac{\pi}{24}\left(X^{3}-18 X^{2} Y+42 X Y^{2}+Y^{3}\right)- \\
& \left.-\frac{\pi^{2}}{48}\left(3 X^{2}+12 X Y+19 Y^{2}+6 i \pi(X+5 Y)\right)+\frac{103}{720} \pi^{4}\right]- \\
& -3\left(2 \frac{y^{2}}{x}-x+5\right)\left[\operatorname{Li}_{3}(-y)+\zeta_{3}-\tilde{Y} \operatorname{Li}_{2}(-y)+i \frac{\pi}{4}\left(Y^{2}+\pi^{2}\right)+\right. \\
& \left.+\frac{1}{12}\left(3 Y^{2}+\pi^{2}\right) Y-X\left(\tilde{Y}^{2}+3 \pi^{2}\right)\right]- \\
& -3\left(4 \frac{y^{2}}{x}-2(1-y)+3 \frac{x^{2}}{y}\right)\left[\operatorname{Li}_{3}(-x)-\zeta_{3}-\tilde{X} \operatorname{Li}_{2}(-x)+\right. \\
& \left.+i \frac{\pi}{2} X(X-i \pi)+i \frac{\pi^{3}}{6}\right]-
\end{aligned}
$$




$$
\begin{aligned}
& -\frac{1}{4}\left[\left(27 \tilde{X}^{2}-18(X-Y)^{2}+17 \pi^{2}\right) \tilde{Y}+232 \pi^{2} X-\right. \\
& \left.\quad-4 i \pi^{3}-48 \zeta_{3}\right]+\frac{1}{2}\left(4 \frac{x^{2}}{y}-x+y\right)\left(\tilde{X}^{3}+\pi^{2}(7 X+i \pi)\right)- \\
& -\frac{y}{4}\left[3\left(2 X^{2}-Y^{2}\right) Y+3 i \pi\left(2 X^{2}+4 X Y-3 Y^{2}\right)+24 \zeta_{3}+\right. \\
& \left.\quad+\pi^{2}(20 X-Y+i \pi)\right]+\frac{3}{8}(2-x)\left(3 \frac{y}{x}-\frac{4}{y}\right)\left(\tilde{X}^{2}+\pi^{2}\right)- \\
& -\frac{3}{8}\left(\frac{8}{x}+y+4 x\right)\left(\tilde{Y}^{2}+\pi^{2}\right)+\frac{27}{8} \pi^{2} y- \\
& -\frac{3}{8}\left(6 \frac{y}{x}+y-12 x\right)\left((X-Y)^{2}+\pi^{2}\right) .
\end{aligned}
$$

\section{References}

[1] F. Aversa, P. Chiappetta, M. Greco and J.P. Guillet, "Higher order corrections to QCD jets," Phys. Lett. B 210, 225 (1988); "Jet production in hadronic collisions to $\mathcal{O}\left(\alpha_{s}^{3}\right)$," Z. Phys. C 46, 253 (1990).

[2] S.D. Ellis, Z. Kunszt and D.E. Soper, "The one-jet inclusive cross section at $\mathcal{O}\left(\alpha_{s}^{3}\right)$ : Quarks And Gluons," Phys. Rev. Lett. 64, 2121 (1990); "Two jet production in hadron collisions at $\mathcal{O}\left(\alpha_{s}^{3}\right)$ in QCD," Phys. Rev. Lett. 69, 1496 (1992).

[3] W.T. Giele, E.W.N. Glover and D.A. Kosower, "Higher order corrections to jet cross sections in hadron colliders," Nucl. Phys. B 403, 633 (1993) [arXiv:hep-ph/9302225].

[4] N. Kidonakis, "Resummation for heavy quark and jet cross sections," Int. J. Mod. Phys. A 15, 1245 (2000) [arXiv:hep-ph/9902484];

N. Kidonakis and J.F. Owens, "Effects of higher-order threshold corrections in high- $E_{T}$ jet production," Phys. Rev. D 63, 054019 (2001) [arXiv:hep-ph/0007268].

[5] S. Alekhin, "Extraction of parton distributions and $\alpha_{s}$ from DIS data within the Bayesian treatment of systematic errors," Eur. Phys. J. C 10, 395 (1999) [arXiv:hep-ph/9611213]; W.T. Giele, S.A. Keller and D.A. Kosower, "Parton distributions with errors," in La Thuile 1999, Results and perspectives in particle physics;

D. Stump et al., "Uncertainties of predictions from parton distribution functions. I: The Lagrange multiplier method," Phys. Rev. D 65, 014012 (2002) [arXiv:hep-ph/0101051]; "Uncertainties of predictions from parton distribution functions. II: The Hessian method," Phys. Rev. D 65, 014013 (2002) [arXiv:hep-ph/0101032];

W.T. Giele, S.A. Keller and D.A. Kosower, "Parton distribution function uncertainties," arXiv:hep-ph/0104052.

[6] A.D. Martin, R.G. Roberts, W.J. Stirling and R.S. Thorne, "Estimating the effect of NNLO contributions on global parton analyses," Eur. Phys. J. C 18, 117 (2000); [arXiv:hep-ph/0007099]; "NNLO global parton analysis," arXiv:hep-ph/0201127.

[7] W.L. van Neerven and A. Vogt, "NNLO evolution of deep-inelastic structure functions: The non-singlet case," Nucl. Phys. B 568, 263 (2000) [arXiv:hep-ph/9907472]; "NNLO evolution of deep-inelastic structure functions: The singlet case," Nucl. Phys. B 588, 345 (2000) 
[arXiv:hep-ph/0006154]; "Improved approximations for the three-loop splitting functions in QCD," Phys. Lett. B 490, 111 (2000) [arXiv:hep-ph/0007362]

[8] F.A. Berends and W. Giele, "The six gluon process as an example of Weyl-Van Der Waerden spinor calculus," Nucl. Phys. B 294, 700 (1987);

M.L. Mangano, S. Parke and Z. Xu, "Duality and multi-gluon scattering," Nucl. Phys. B 298, 653 (1988).

[9] M.L. Mangano and S.J. Parke, "Multiparton amplitudes in gauge theories," Phys. Rept. 200, 301 (1991).

[10] Z. Bern, L.J. Dixon and D.A. Kosower, "One-loop corrections to five-gluon amplitudes," Phys. Rev. Lett. 70, 2677 (1993) [arXiv:hep-ph/9302280];

Z. Kunszt, A. Signer and Z. Trócsányi, "One-loop radiative corrections to the helicity amplitudes of QCD processes involving four quarks and one gluon," Phys. Lett. B 336, 529 (1994) [arXiv:hep-ph/9405386];

Z. Bern, L.J. Dixon and D.A. Kosower, "One-loop corrections to two-quark three-gluon amplitudes," Nucl. Phys. B 437, 259 (1995) [arXiv:hep-ph/9409393].

[11] C. Anastasiou, E.W.N. Glover, C. Oleari and M.E. Tejeda-Yeomans, "Two-loop QCD corrections to $q \bar{q} \rightarrow q^{\prime} \bar{q}^{\prime}$," Nucl. Phys. B 601, 318 (2001) [hep-ph/0010212];

"Two-loop QCD corrections to $q \bar{q} \rightarrow q \bar{q}$, " Nucl. Phys. B 601, 341 (2001) [hep-ph/0011094];

"Two-loop QCD corrections to massless quark gluon scattering," Nucl. Phys. B 605, 486

(2001) [hep-ph/0101304].

[12] E.W.N. Glover, C. Oleari and M.E. Tejeda-Yeomans, "Two-loop QCD corrections to gluon gluon scattering," Nucl. Phys. B 605, 467 (2001) [arXiv:hep-ph/0102201].

[13] F.A. Berends, R. Kleiss, P. De Causmaecker, R. Gastmans and T.T. Wu, "Single bremsstrahlung processes in gauge theories," Phys. Lett. B 103, 124 (1981);

P. De Causmaecker, R. Gastmans, W. Troost and T.T. Wu, "Helicity amplitudes for massless QED," Phys. Lett. B 105, 215 (1981);

Z. Xu, D. Zhang and L. Chang, "Helicity amplitudes for multiple bremsstrahlung in massless nonabelian gauge theories," Nucl. Phys. B 291, 392 (1987).

[14] J. Soffer and J.M. Virey, "Testing various polarized parton distributions at RHIC," Nucl. Phys. B 509, 297 (1998) [arXiv:hep-ph/9706229].

[15] D. de Florian, S. Frixione, A. Signer and W. Vogelsang, "Next-to-leading order jet cross sections in polarized hadronic collisions," Nucl. Phys. B 539, 455 (1999) [arXiv:hep-ph/9808262].

[16] M.T. Grisaru, H.N. Pendleton and P. van Nieuwenhuizen, "Supergravity and the S matrix," Phys. Rev. D 15, 996 (1977);

M.T. Grisaru and H.N. Pendleton, "Some properties of scattering amplitudes in supersymmetric theories," Nucl. Phys. B 124, 81 (1977);

S.J. Parke and T.R. Taylor, "Perturbative QCD utilizing extended supersymmetry," Phys. Lett. B 157, 81 (1985), err. ibid. 174B, 465 (1985).

[17] Z. Bern, L. Dixon, D.C. Dunbar and D.A. Kosower, "One-loop n-point gauge theory amplitudes, unitarity and collinear limits," Nucl. Phys. B425, 217 (1994) [arXiv:hep-ph/9403226].

[18] Z. Bern, L. Dixon and D.A. Kosower, "Progress in one-loop QCD computations," Ann. Rev. Nucl. Part. Sci. 46, 109 (1996) [arXiv:hep-ph/9602280]. 
[19] E.A. Kuraev, L.N. Lipatov and V.S. Fadin, "Multi-reggeon processes in the Yang-Mills theory," Sov. Phys. JETP 44, 443 (1976) [Zh. Eksp. Teor. Fiz. 71, 840 (1976)];

[20] J.C. Collins, Renormalization: an introduction to renormalization group, and the operator-product expansion, Cambridge Monographs on Mathematical Physics (Cambridge Univ. Press, 1984).

[21] G. 't Hooft and M. Veltman, "Regularization and renormalization of gauge fields," Nucl. Phys. B44, 189 (1972).

[22] Z. Bern and D.A. Kosower, "The computation of loop amplitudes in gauge theories," Nucl. Phys. B379, 451 (1992).

[23] Z. Bern, A. De Freitas, L. Dixon and H.L. Wong, "Supersymmetric regularization, two-loop QCD amplitudes and coupling shifts," SLAC-PUB-9104, UCLA/02/TEP/2.

[24] W. Siegel, "Supersymmetric dimensional regularization via dimensional reduction," Phys. Lett. B84, 193 (1979);

D.M. Capper, D.R.T. Jones and P. van Nieuwenhuizen, "Regularization by dimensional reduction of supersymmetric and nonsupersymmetric gauge theories," Nucl. Phys. B167, 479 (1980). I. Jack, D.R.T. Jones and K.L. Roberts, "Equivalence of dimensional reduction and dimensional regularization," Z. Phys. C63, 151 (1994) [arXiv:hep-ph/9401349].

[25] S. Catani, "The singular behaviour of QCD amplitudes at two-loop order," Phys. Lett. B427, 161 (1998) [arXiv:hep-ph/9802439].

[26] S. Catani and M. Grazzini, "The soft-gluon current at one-loop order," Nucl. Phys. B 591, 435 (2000) [arXiv:hep-ph/0007142].

[27] Z. Bern and A.G. Morgan, "Massive loop amplitudes from unitarity," Nucl. Phys. B 467, 479 (1996) [arXiv:hep-ph/9511336].

[28] Z. Bern, L.J. Dixon, D.C. Dunbar and D.A. Kosower, "One-loop self-dual and $N=4$ super-Yang-Mills," Phys. Lett. B 394, 105 (1997) [arXiv:hep-th/9611127].

[29] Z. Bern, V. Del Duca and C.R. Schmidt, "The infrared behavior of one-loop gluon amplitudes at next-to-next-to-leading order," Phys. Lett. B 445, 168 (1998) [arXiv:hep-ph/9810409].

[30] L.W. Garland, T. Gehrmann, E.W.N. Glover, A. Koukoutsakis and E. Remiddi, "The two-loop QCD matrix element for $e^{+} e^{-} \rightarrow 3$ jets," arXiv:hep-ph/0112081.

[31] E.W.N. Glover and M. E. Tejeda-Yeomans, "One-loop QCD corrections to gluon-gluon scattering at NNLO," JHEP 0105, 010 (2001) [arXiv:hep-ph/0104178].

[32] R.K. Ellis and J.C. Sexton, "QCD Radiative corrections to parton-parton scattering," Nucl. Phys. B 269, 445 (1986).

[33] Z. Kunszt, A. Signer and Z. Trócsányi, "One loop helicity amplitudes for all $2 \rightarrow 2$ processes in QCD and $N=1$ supersymmetric Yang-Mills theory," Nucl. Phys. B411, 397 (1994) [arXiv:hep-ph/9305239].

[34] Z. Bern, L. Dixon and D.A. Kosower, "A two-loop four-gluon helicity amplitude in QCD," JHEP 0001, 027 (2000) [arXiv:hep-ph/0001001].

[35] Z. Bern, L.J. Dixon and A. Ghinculov, "Two-loop correction to Bhabha scattering," Phys. Rev. D 63, 053007 (2001) [arXiv:hep-ph/0010075]. 
[36] Z. Bern and D.A. Kosower, "Color decomposition of one-loop amplitudes in gauge theories," Nucl. Phys. B 362, 389 (1991).

[37] Z. Bern, L. Dixon, D.C. Dunbar and D.A. Kosower, "Fusing gauge theory tree amplitudes into loop amplitudes," Nucl. Phys. B 435, 59 (1995) [arXiv:hep-ph/9409265].

[38] Z. Bern, L. Dixon and D.A. Kosower, "Dimensionally regulated one loop integrals," Phys. Lett. B 302, 299 (1993), err. ibid. B 318, 649 (1993) [arXiv:hep-ph/9212308];

Z. Bern, L. Dixon and D.A. Kosower, "Dimensionally regulated pentagon integrals," Nucl. Phys. B 412, 751 (1994) [arXiv:hep-ph/9306240].

[39] K. Fabricius and I. Schmitt, Z. Phys. C 3, 51 (1979);

S. Papadopoulos, A. P. Contogouris and J. Ralston, Phys. Rev. D 25, 2218 (1982).

[40] Z. Bern and G. Chalmers, "Factorization in one loop gauge theory," Nucl. Phys. B 447, 465 (1995) [arXiv:hep-ph/9503236].

[41] D. A. Kosower and P. Uwer, "One-loop splitting amplitudes in gauge theory," Nucl. Phys. B 563, 477 (1999) [arXiv:hep-ph/9903515];

Z. Bern, V. Del Duca, W.B. Kilgore and C.R. Schmidt, "The infrared behavior of one-loop QCD amplitudes at next-to-next-to-leading order," Phys. Rev. D 60, 116001 (1999) [arXiv:hep-ph/9903516].

[42] S. Catani and M.H. Seymour, "A general algorithm for calculating jet cross sections in NLO QCD," Nucl. Phys. B 485, 291 (1997), err. ibid. B 510, 503 (1997) [arXiv:hep-ph/9605323].

[43] W.L. van Neerven, "Dimensional regularization of mass and infrared singularities in two-loop on-shell vertex functions," Nucl. Phys. B268, 453 (1986).

[44] Z. Bern, J.S. Rozowsky and B. Yan, "Two-loop four-gluon amplitudes in $N=4$ super-Yang-Mills," Phys. Lett. B 401, 273 (1997) [arXiv:hep-ph/9702424];

Z. Bern, L. Dixon, D.C. Dunbar, M. Perelstein and J.S. Rozowsky, "On the relationship between Yang-Mills theory and gravity and its implication for ultraviolet divergences," Nucl. Phys. B 530, 401 (1998) [arXiv:hep-th/9802162].

[45] V.A. Smirnov and O.L. Veretin, "Analytical results for dimensionally regularized massless on-shell double boxes with arbitrary indices and numerators," Nucl. Phys. B566, 469 (2000) [arXiv:hep-ph/9907385].

[46] C. Anastasiou, E.W.N. Glover and C. Oleari, "Application of the negative-dimension approach to massless scalar box integrals," Nucl. Phys. B565, 445 (2000) [arXiv:hep-ph/9907523];

C. Anastasiou, E.W.N. Glover and C. Oleari, "The two-loop scalar and tensor pentabox graph with light-like legs," Nucl. Phys. B575, 416 (2000), err. ibid. B585, 763 (2000) [arXiv:hep-ph/9912251].

[47] C. Anastasiou, T. Gehrmann, C. Oleari, E. Remiddi and J.B. Tausk, "The tensor reduction and master integrals of the two-loop massless crossed box with light-like legs," Nucl. Phys. B580, 577 (2000) [arXiv:hep-ph/0003261].

[48] T. Gehrmann and E. Remiddi, "Differential equations for two-loop four-point functions," Nucl. Phys. B 580, 485 (2000) [arXiv:hep-ph/9912329].

[49] Z. Bern, A. De Freitas and L.J. Dixon, "Two-loop amplitudes for gluon fusion into two photons," JHEP 0109, 037 (2001) [arXiv:hep-ph/0109078]. 
[50] Z. Bern, A. De Freitas, L.J. Dixon, A. Ghinculov and H. L. Wong, "QCD and QED corrections to light-by-light scattering," JHEP 0111, 031 (2001) [arXiv:hep-ph/0109079].

[51] F.V. Tkachov, "A theorem on analytical calculability of four-loop renormalization group functions," Phys. Lett. B 100, 65 (1981);

K.G. Chetyrkin and F.V. Tkachov, "Integration by parts: the algorithm to calculate beta functions in 4 loops," Nucl. Phys. B 192, 159 (1981).

[52] O.V. Tarasov, Phys. Rev. D 54, 6479 (1996) [arXiv:hep-th/9606018].

[53] V.A. Smirnov, "Analytical result for dimensionally regularized massless on-shell double box," Phys. Lett. B460, 397 (1999) [arXiv:hep-ph/9905323].

[54] J.B. Tausk, "Non-planar massless two-loop Feynman diagrams with four on-shell legs," Phys. Lett. B469, 225 (1999) [arXiv:hep-ph/9909506].

[55] See e.g. K.S. Kölbig, "Nielsen's generalized polylogarithms," SIAM J. Math. Anal. 17, 1232 (1986).

[56] K.S. Kölbig, J.A. Mignaco and E. Remiddi, B.I.T. 10, 38 (1970).

[57] L. Lewin, Dilogarithms and Associated Functions (Macdonald, 1958).

[58] C. Anastasiou, private communication.

[59] D.A. Kosower, "The spinor helicity method in dimensional regularization," Phys. Lett. B 254, 439 (1991).

[60] S. Catani, M.H. Seymour and Z. Trócsányi, "Regularization scheme independence and unitarity in QCD cross sections," Phys. Rev. D55, 6819 (1997) [arXiv:hep-ph/9610553].

[61] G. Altarelli, G. Curci, G. Martinelli and S. Petrarca, "QCD nonleading corrections to weak decays as an application of regularization by dimensional reduction," Nucl. Phys. B 187, 461 (1981);

I. Antoniadis, C. Kounnas and K. Tamvakis, "Simple treatment of threshold effects," Phys. Lett. B 119, 377 (1982);

S.P. Martin and M.T. Vaughn, "Regularization dependence of running couplings in softly broken supersymmetry," Phys. Lett. B 318, 331 (1993) [arXiv:hep-ph/9308222].

[62] O.V. Tarasov, A.A. Vladimirov and A.Y. Zharkov, "The Gell-Mann-Low function of QCD in the three loop approximation," Phys. Lett. B 93, 429 (1980);

S.A. Larin and J.A.M. Vermaseren, "The three loop QCD beta function and anomalous dimensions," Phys. Lett. B 303, 334 (1993) [arXiv:hep-ph/9302208].

[63] J.F. Gunion and Z. Kunszt, "Improved analytic techniques for tree graph calculations and the $g g q \bar{q} \ell \bar{\ell}$ subprocess," Phys. Lett. B 161, 333 (1985). 